Louisiana State University

LSU Digital Commons

Faculty Publications

Department of Mathematics

$5-1-2021$

\title{
Nonlocal elastodynamics and fracture
}

Robert P. Lipton

Louisiana State University

Prashant K. Jha

The University of Texas at Austin

Follow this and additional works at: https://digitalcommons.Isu.edu/mathematics_pubs

\section{Recommended Citation}

Lipton, R., \& Jha, P. (2021). Nonlocal elastodynamics and fracture. Nonlinear Differential Equations and Applications, 28 (3) https://doi.org/10.1007/s00030-021-00683-x

This Article is brought to you for free and open access by the Department of Mathematics at LSU Digital Commons. It has been accepted for inclusion in Faculty Publications by an authorized administrator of LSU Digital Commons. For more information, please contact ir@lsu.edu. 


\title{
Nonlocal elastodynamics and fracture
}

\author{
Robert P. Lipton® and Prashant K. Jha@
}

\begin{abstract}
A nonlocal field theory of peridynamic type is applied to model the brittle fracture problem. The elastic fields obtained from the nonlocal model are shown to converge in the limit of vanishing non-locality to solutions of classic plane elastodynamics associated with a running crack. We carry out our analysis for a plate subject to mode one loading. The length of the crack is prescribed a priori and is an increasing function of time.
\end{abstract}

Mathematics Subject Classification. 34A99, 74R99.

Keywords. Brittle fracture, Peridynamics, Nonlinear, Nonlocal, Elastodynamic.

\section{Introduction}

Fracture can be viewed as a collective interaction across large and small length scales. With the application of enough stress or strain to a brittle material, atomistic scale bonds will break, leading to fracture of the macroscopic specimen. From a modeling perspective fracture should appear as an emergent phenomena generated by an underlying field theory eliminating the need for a supplemental kinetic relation describing crack growth. The displacement field inside the body for points $\boldsymbol{x}$ at time $t$ is written $\boldsymbol{u}(\boldsymbol{x}, t)$. The peridynamic model $[35,36]$, is described by the nonlocal balance of linear momentum of the form

$$
\rho \boldsymbol{u}_{t t}(\boldsymbol{x}, t)=\int_{\mathcal{H}_{\epsilon}(\boldsymbol{x})} \boldsymbol{f}(\boldsymbol{y}, \boldsymbol{x}) d \boldsymbol{y}+\boldsymbol{b}(\boldsymbol{x}, t)
$$

where $\mathcal{H}_{\epsilon}(\boldsymbol{x})$ is a neighborhood of $\boldsymbol{x}, \rho$ is the density, $\boldsymbol{b}$ is the body force density field, and $\boldsymbol{f}$ is a material-dependent constitutive law that represents the force density that a point $\boldsymbol{y}$ inside the neighborhood exerts on $\boldsymbol{x}$ as a result of the deformation field. The radius $\epsilon$ of the neighborhood is referred

This material is based upon work supported by the U. S. Army Research Laboratory and the U. S. Army Research Office under Contract/Grant Number W911NF-19-1-0245. 


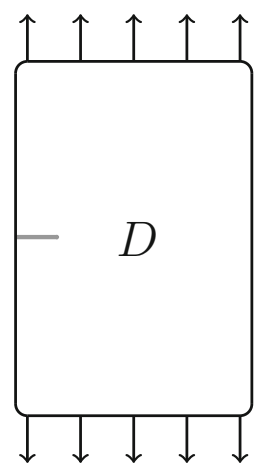

Figure 1. Plate with initial crack on the left edge

to as the horizon. Here all points satisfy the same field equation (1.1). The displacement fields and fracture evolution predicted by the nonlocal model should agree with the dynamic fracture of specimens when the length scale of non-locality is sufficiently small. In this respect numerical simulations are compelling, see for example [4,37], and [39].

The displacement for the nonlocal theory is examined in the limit of vanishing non-locality. This is done for a class of peridynamic models with nonlocal forces derived from double well potentials see, [24]. The term double well describes the force potential between two points. One of the wells is degenerate and appears at infinity while the other is at zero strain. For small strains the nonlocal force is linearly elastic but for larger strains the force begins to soften and then approaches zero after reaching a critical strain. This type of nonlocal model is called a cohesive model. Fracture energies of this type have been defined for displacement gradients in [40] with the goal of understanding fracture as a phase transition in the framework of [14].

We theoretically investigate the limit of the displacements for the cohesive model as the length scale $\epsilon$ of nonlocal interaction goes to zero. All information on this limit is obtained from what is known from the nonlocal model for $\epsilon>0$. In this paper the single edge notch specimen is considered as given in Fig. 1 and the target theory governing the evolution of displacement fields is identified when $\epsilon=0$.

One of the hallmarks of peridynamic simulations is localization of defect sets with horizon as $\epsilon \rightarrow 0$. Theoretically localization of the jump set of the displacement is established as $\epsilon \rightarrow 0$ in $[23,24]$ where the limiting displacement is shown to be an $S B D^{2}(D)$ valued function for almost all times $t \in[0, T]$, see Sect. 3. The nonlocal cohesive model converges to a dynamic model having bounded Griffith fracture energy associated with brittle fracture and elastic displacement fields satisfying the elastic wave equation $[23,24]$ away from the fractures. This can be seen for arbitrarily shaped specimens with smooth boundary in two and three dimensions. However the explicit traction law relating the crack boundary to the elastic field lies out side the scope of that analysis. 
This paper builds on earlier work and provides a global description of the limit dynamics describing elastic fields surrounding a crack for the single edge notch pulled apart by traction forces on its top and bottom edges. In this treatment the advancing crack is prescribed as a failure zone in the neighborhood of a line at the center of a rectangular specimen starting from a notch. Symmetric forces and boundary conditions are imposed, consistent with the assumption of a crack growing on a line and moving into the specimen. The length of the failure zone is prescribed a priori and is an increasing function of time for the nonlocal model. This paper does not investigate crack initiation, and the crack grows from an initial crack. The objective of this paper is to show that the elastic fields seen in the nonlocal model are consistent with those in the local model in the limit of vanishing horizon. The analysis given here shows that it is possible to recover the boundary value problem for the linear elastic displacement given by Linear Elastic Fracture Mechanics inside a cracking body as the limit of a nonlocal fracture model. To illustrate this a family of initial value problems given in the nonlocal formulation is prescribed. The family is parameterized by horizon size $\epsilon$. The crack motion for $\epsilon>0$ is prescribed by the solutions of the nonlocal initial value problem. It is shown that up to subsequences, as $\epsilon \rightarrow 0$, the displacements associated with the solution of the nonlocal model converge in mean square uniformly in time to the limit displacement $\boldsymbol{u}^{0}(\boldsymbol{x}, t)$ that satisfies:

- Prescribed inhomogeneous traction boundary conditions.

- Balance of linear momentum as described by the linear elastic wave equation off the crack.

- Zero traction on the sides of the evolving crack.

- The set on which the elastic displacement jumps is a subset of the crack set.

- The limiting elastodynamics is determined by the sequence of nonlocal problems for $\epsilon>0$ and is obtained in the $\epsilon=0$ limit.

The first four items deliver the boundary conditions, elastodynamic equations, location of the jump set of the elastic field, and traction boundary conditions on the crack. The $\epsilon \rightarrow 0$ limit of displacement fields for the nonlocal model is seen to be a weak solution for the wave equation on a time dependent domain recently defined in the work of [12], see theorem 3.4. Here the time dependent domain is given by the domain surrounding the moving crack. This establishes a rigorous connection between the nonlocal fracture formulation using a peridynamic model derived from a double well potential and the wave equation posed on cracking domains given in [12].

Peridynamic modeling implicitly couples the dynamics of the un-cracked elastic material to crack tip growth. Although it is beyond the scope of the current paper, it is of natural interest to explicitly recover the coupling between the length of the crack and the dynamics of the elastic field. One answer lies in deriving an explicit formula relating the time rate of change of kinetic energy and stress work inside a neighborhood surrounding the crack tip to the external elastic power applied to the neighborhood. This formula is obtained by multiplying the equation of motion (2.16) by the velocity field, then performing 
an integration by parts, see [22]. With this formula in hand, one can formally examine the $\epsilon \rightarrow 0$ case to identify the coupling between the motion of the crack tip and the surrounding elastic field of the intact material, see [22]. For example, when the change in internal energy inside a vanishingly small neighborhood of the crack tip is zero, then the crack velocity is coupled to the dynamics of the elastic field by the kinetic relation of dynamic Linear Elastic Fracture Mechanics (LEFM) [3,17,32,38], see [22]. Another approach that address sharp free crack motion directly is recently developed in [11]. That approach provides a well posed sharp fracture model that couples the elastic dynamics of intact material to the evolving crack tip motion over an interval of crack velocities. That model provides for non-stationary free crack motion based on a weak maximal dissipation condition and energy dissipation balance [11].

The analysis given here treats a dynamic problem and compactness methods suited to the balance of momentum for nonlocal - nonlinear operators, are applied, see Lemma 3.2 and Theorem 3.2. Proceeding this way delivers the zero traction condition on the crack lips for the fracture model in the local limit. Another issue is to prescribe body forces for the nonlocal model that transform to into boundary tractions for the local model. In this paper a suitable layer of force is prescribed adjacent to the boundary of the sample for the nonlocal model. It is motivated by the one proposed in [39]. The layer of force is shown to converge to the standard traction boundary conditions seen in local models, see Lemma 3.1. This theoretically corroborates the numerical experiments with the nonlocal model carried out in [39]. It is pointed out that the nonlocal model considered here is elastic, so cracks can heal if the strain across the crack drops below the critical value. However, in this paper the initial conditions and boundary conditions are chosen such that the specimen is under tensile strain and pulled apart so the crack has no opportunity to heal. More complex models [26] involving dissipation and non-monotone or cyclic load paths lie outside the scope of the paper and provide interesting avenues for future research.

The nonlocal model is an example of several new approaches to dynamic fracture modeling. These include solution of the wave equation on cracking domains $[9,10,12,31]$, phase field methods, $[6,7,29,34]$, and peridynamics $[4,35,36,39]$. In the absence of fracture and dynamics the $\Gamma$ convergence approach has been applied to peridynamic boundary value problems. The nonlocal formulations are shown to converge to equilibrium boundary value problems for hyperelastic and elastic materials as $\epsilon \rightarrow 0$, see [5,30]. It is noted that the aforementioned references while relevant to this work are only a few from a rapidly expanding literature.

The paper is organized as follows: In Sect. 2 the nonlocal constitutive law as derived from a double well potential is described and the nonlocal boundary value problem describing crack evolution is given. Section 3 provides the principle results of the paper and describes the convergence of the displacement fields given in the nonlocal model to the elastic displacement field satisfying, the linear wave equation off the crack set, zero Neumann conditions on the 
crack, and traction boundary conditions. Existence and uniqueness for the nonlocal problems are established in 4 . The convergence theorems are proved in Sects. 5 and 6. The proof that the limit displacement is a weak solution of the wave equation on a time dependent domain is given in Sect. 7. The results are summarized in the conclusion Sect. 8.

\section{Nonlocal elastodynamics}

In this section we formulate the nonlocal dynamics as an initial boundary value problem driven by a layer of force adjacent to the boundary. Here all quantities are non-dimensional. Define the region $D$ given by a rectangle with rounded corners, see Fig. 1. The domain lies within the rectangle $\left\{0<x_{1}<\right.$ $\left.a ;-b / 2<x_{2}<b / 2\right\}$ and the initial crack originates on the left side of the specimen, see Fig. 1. The specific dimensions of the initial crack are given in Sect. 2.2. The domain is subject to plane strain loading and we will assume small deformations so the deformed configuration is the same as the reference configuration. We have $\boldsymbol{u}=\boldsymbol{u}(\boldsymbol{x}, t)$ as a function of space and time but will suppress the $\boldsymbol{x}$ dependence when convenient and write $\boldsymbol{u}(t)$. The tensile strain $S$ between two points $\boldsymbol{x}, \boldsymbol{y}$ in $D$ along the direction $\boldsymbol{e}_{\boldsymbol{y}-\boldsymbol{x}}$ is defined as

$$
S(\boldsymbol{y}, \boldsymbol{x}, \boldsymbol{u}(t))=\frac{\boldsymbol{u}(\boldsymbol{y}, t)-\boldsymbol{u}(\boldsymbol{x}, t)}{|\boldsymbol{y}-\boldsymbol{x}|} \cdot \boldsymbol{e}_{\boldsymbol{y}-\boldsymbol{x}},
$$

where $\boldsymbol{e}_{\boldsymbol{y}-\boldsymbol{x}}=\frac{\boldsymbol{y}-\boldsymbol{x}}{|\boldsymbol{y}-\boldsymbol{x}|}$ is a unit vector and "." is the dot product.

The nonlocal force $\boldsymbol{f}$ is defined in terms of a double well potential that is a function of the strain $S(\boldsymbol{y}, \boldsymbol{x}, \boldsymbol{u}(t))$. We define

$$
\mathcal{W}^{\epsilon}(\boldsymbol{z}, S)=J^{\epsilon}(|\boldsymbol{z}|) \frac{1}{\epsilon^{3} \omega_{2}|\boldsymbol{z}|} \Psi(\sqrt{|\boldsymbol{z}|} S)
$$

The force potential is defined for all $\boldsymbol{x}, \boldsymbol{y}$ in $D$ by

$$
\mathcal{W}^{\epsilon}(\boldsymbol{y}-\boldsymbol{x}, S(\boldsymbol{y}, \boldsymbol{x}, \boldsymbol{u}(t)))=J^{\epsilon}(|\boldsymbol{y}-\boldsymbol{x}|) \frac{1}{\epsilon^{3} \omega_{2}|\boldsymbol{y}-\boldsymbol{x}|} \Psi(\sqrt{|\boldsymbol{y}-\boldsymbol{x}|} S(\boldsymbol{y}, \boldsymbol{x}, \boldsymbol{u}(t)))
$$

where $\mathcal{W}^{\epsilon}(\boldsymbol{y}-\boldsymbol{x}, S(\boldsymbol{y}, \boldsymbol{x}, \boldsymbol{u}(t)))$ is the pairwise force potential per unit length between two points $\boldsymbol{x}$ and $\boldsymbol{y}$. Here, the influence function $J^{\epsilon}(|\boldsymbol{y}-\boldsymbol{x}|)$ is a measure of the influence that the point $\boldsymbol{y}$ has on $\boldsymbol{x}$. Only points inside the horizon can influence $\boldsymbol{x}$ so $J^{\epsilon}(|\boldsymbol{y}-\boldsymbol{x}|)$ is nonzero for $|\boldsymbol{y}-\boldsymbol{x}|<\epsilon$ and is zero otherwise. We take $J^{\epsilon}$ to be of the form: $J^{\epsilon}(|\boldsymbol{y}-\boldsymbol{x}|)=J\left(\frac{|\boldsymbol{y}-\boldsymbol{x}|}{\epsilon}\right)$ with $J(r)=0$ for $r \geq 1$ and $0 \leq J(r) \leq M<\infty$ for $r<1$.

The scale factor of $\epsilon^{-3}$ is chosen in (2.3) following [24] so that:

1. The elastic constants associated with the $\epsilon=0$ limit are given by (5.6).

2. The energy release rate per unit crack length is independent of $\epsilon$ and given by (5.7).

Item (1) is invoked to find the dynamic evolution of the elastic field off the crack in Sect. 3. Item (2) is used in an estimate to show the $\epsilon=0$ of the displacement lies in the space of Special Functions of bounded Deformation, see (5.8). 


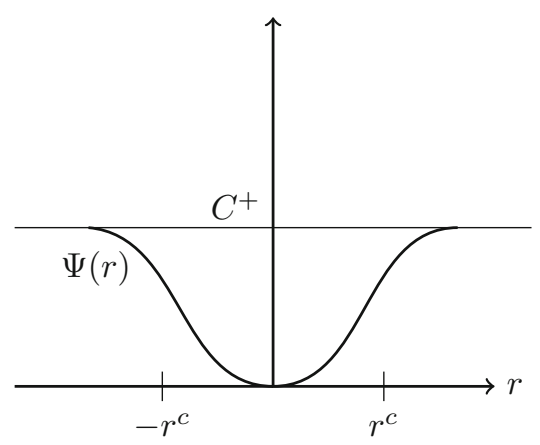

(a)

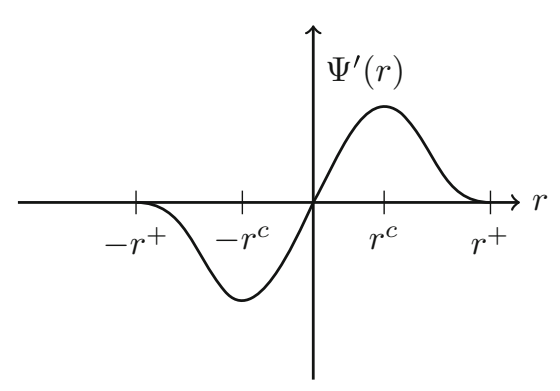

(b)

FiguRE 2. a The double well potential function $\Psi(r)$ for tensile force. Here $C^{+}$is the asymptotic value of $\Psi$. b Cohesive force. The derivative of the force potential goes smoothly to zero at $\pm r^{+}$

The force potential is described in terms of its potential function and to fix ideas $\Psi$ is given by

$$
\Psi=h\left(r^{2}\right)
$$

where $h$ is concave, see Fig. 2a. Here $\omega_{2}$ is the area of the unit disk and $\epsilon^{2} \omega_{2}$ is the area of the horizon $\mathcal{H}_{\epsilon}(\boldsymbol{x})$. The potential function $\Psi$ represents a convexconcave potential such that the associated force acting between material points $\boldsymbol{x}$ and $\boldsymbol{y}$ are initially elastic and then soften and decay to zero as the strain between points increases, see Fig. 2b. The force between $\boldsymbol{x}$ and $\boldsymbol{y}$ is referred to as the bond force. The first well for $\mathcal{W}^{\epsilon}(\boldsymbol{x}-\boldsymbol{y}, S(\boldsymbol{y}, \boldsymbol{x}, \boldsymbol{u}(t)))$ is at zero tensile strain and the potential function satisfies

$$
\Psi(0)=\Psi^{\prime}(0)=0
$$

The well for $\mathcal{W}^{\epsilon}(\boldsymbol{y}-\boldsymbol{x}, S(\boldsymbol{y}, \boldsymbol{x}, \boldsymbol{u}(t)))$ in the neighborhood of infinity is characterized by the horizontal asymptote $\lim _{S \rightarrow \infty} \Psi(S)=C^{+}$, see Fig. 2a. The critical tensile strain $S_{c}>0$ for which the force begins to soften is given by the inflection point $r^{c}>0$ of $g$ and is

$$
S_{c}=\frac{r^{c}}{\sqrt{|\boldsymbol{y}-\boldsymbol{x}|}},
$$

and $S_{+}$is the strain at which the force goes to zero

$$
S_{+}=\frac{r^{+}}{\sqrt{|\boldsymbol{y}-\boldsymbol{x}|}} .
$$

We assume here that the potential functions are bounded and are smooth. It is pointed out that for this modeling the bond force in compression allows for eventual softening. However one can easily generalize the analysis to handle an asymmetric bond force that resists compression. 


\subsection{Peridynamic equation of motion}

The potential energy of the motion is given by

$$
P D^{\epsilon}(\boldsymbol{u})=\int_{D} \int_{\mathcal{H}_{\epsilon}(\boldsymbol{x}) \cap D}|\boldsymbol{y}-\boldsymbol{x}| \mathcal{W}^{\epsilon}(\boldsymbol{y}-\boldsymbol{x}, S(\boldsymbol{y}, \boldsymbol{x}, \boldsymbol{u}(t))) d \boldsymbol{y} d \boldsymbol{x} .
$$

We consider single edge notched specimen $D$ pulled apart by an $\epsilon$ thickness layer of body force on the top and bottom of the domain consistent with plain strain loading. In the nonlocal setting the "traction" is given by the layer of body force on the top and bottom of the domain. For this case the body force is written as

$$
\begin{aligned}
& \boldsymbol{b}^{\epsilon}(\boldsymbol{x}, t)=\boldsymbol{e}^{2} \epsilon^{-1} g\left(x_{1}, t\right) \chi_{+}^{\epsilon}\left(x_{1}, x_{2}\right) \text { on the top layer and } \\
& \boldsymbol{b}^{\epsilon}(\boldsymbol{x}, t)=-\boldsymbol{e}^{2} \epsilon^{-1} g\left(x_{1}, t\right) \chi_{-}^{\epsilon}\left(x_{1}, x_{2}\right) \text { on the bottom layer, }
\end{aligned}
$$

where $\boldsymbol{e}^{2}$ is the unit vector in the vertical direction, $\chi_{+}^{\epsilon}$ and $\chi_{-}^{\epsilon}$ are the characteristic functions of the boundary layers given by

$$
\begin{aligned}
& \chi_{+}^{\epsilon}\left(x_{1}, x_{2}\right)=1 \text { on }\left\{\theta<x_{1}<a-\theta, b / 2-\epsilon<x_{2}<b / 2\right\} \text { and 0otherwise, } \\
& \chi_{-}^{\epsilon}\left(x_{1}, x_{2}\right)=1 \text { on }\left\{\theta<x_{1}<a-\theta,-b / 2<x_{2}<-b / 2+\epsilon\right\} \text { and } 0 \text { otherwise, }
\end{aligned}
$$

where $\theta$ is the radius of curvature of the rounded corners of $D$. The top and bottom traction forces are equal and in opposite directions and $g\left(x_{1}, t\right)>0$. We take the function $g$ to be smooth and bounded in the variables $x_{1}:=$ and $t$ and define $\boldsymbol{g}$ on $\partial D$ such that

$$
\boldsymbol{g}= \pm \boldsymbol{e}^{2} g \text { on }\left\{\theta \leq x_{1} \leq a-\theta, x_{2}= \pm b / 2\right\} \text { and } \boldsymbol{g}=0 \text { elsewhere on } \partial D .
$$

The subspace of $L^{2}\left(D, \mathbb{R}^{2}\right)$ given by all rigid body motions $\mathcal{U}$ is defined by

$$
\mathcal{U}=\left\{\boldsymbol{w}: \boldsymbol{w}=\mathbb{Q} \boldsymbol{x}+\mathbf{c} ; \mathbb{Q} \in \mathbb{R}^{2 \times 2}, \mathbb{Q}^{T}=-\mathbb{Q} ; \mathbf{c} \in \mathbb{R}^{2}\right\},
$$

From its definition the body force $\boldsymbol{b}^{\epsilon}(\boldsymbol{x}, t)$ satisfies $\int_{D} \boldsymbol{w} \cdot \boldsymbol{b}^{\epsilon} d \boldsymbol{x}=0$ for all $\boldsymbol{w} \in \mathcal{U}$ and $S(\boldsymbol{y}, \boldsymbol{x}, \boldsymbol{w})=0$ for $\boldsymbol{w} \in \mathcal{U}$. With this in mind we introduce the subspace of $L^{2}\left(D ; \mathbb{R}^{2}\right)$ denoted by

$$
\dot{L}^{2}\left(D ; \mathbb{R}^{2}\right)
$$

defined to be all elements of $L^{2}\left(D ; \mathbb{R}^{2}\right)$ orthogonal to $\mathcal{U}$.

In this treatment the density $\rho$ is assumed constant and we define the Lagrangian

$$
\mathrm{L}\left(\boldsymbol{u}, \partial_{t} \boldsymbol{u}, t\right)=\frac{\rho}{2}\|\dot{\boldsymbol{u}}\|_{L^{2}\left(D ; \mathbb{R}^{2}\right)}^{2}-P D^{\epsilon}(\boldsymbol{u})+\int_{D} \boldsymbol{b}^{\epsilon} \cdot \boldsymbol{u} d \boldsymbol{x},
$$

where $\dot{\boldsymbol{u}}=\frac{\partial \boldsymbol{u}}{\partial t}$ is the velocity. The action integral defined on $\dot{L}^{2}\left(D ; \mathbb{R}^{2}\right)$ for a time evolution over the interval $0<t<T$, is given by

$$
I=\int_{0}^{T} \mathrm{~L}\left(\boldsymbol{u}, \partial_{t} \boldsymbol{u}, t\right) d t
$$




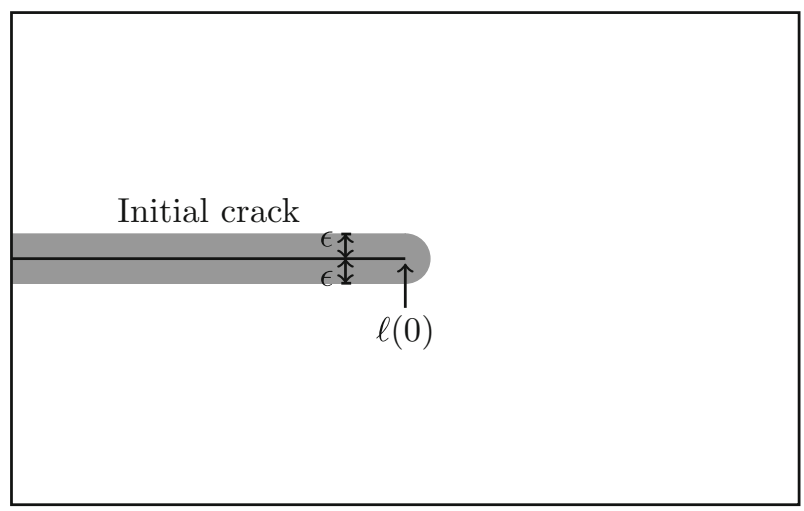

FIgURE 3. The initial crack for the non-local model

We suppose $\boldsymbol{u}^{\epsilon}(t)$ is a stationary point and $\boldsymbol{w}(t)$ is a perturbation and applying the principal of least action gives the nonlocal dynamics

$$
\begin{aligned}
\rho \int_{0}^{T} & \int_{D} \dot{\boldsymbol{u}}^{\epsilon}(\boldsymbol{x}, t) \cdot \dot{\boldsymbol{w}}(\boldsymbol{x}, t) d \boldsymbol{x} d t \\
\quad & \int_{0}^{T} \int_{D} \int_{\mathcal{H}_{\epsilon}(\boldsymbol{x}) \cap D}|\boldsymbol{y}-\boldsymbol{x}| \partial_{S} \mathcal{W}^{\epsilon} \\
& \times\left(\boldsymbol{y}-\boldsymbol{x}, S\left(\boldsymbol{y}, \boldsymbol{x}, \boldsymbol{u}^{\epsilon}(t)\right)\right) S(\boldsymbol{y}, \boldsymbol{x}, \boldsymbol{w}(t)) d \boldsymbol{y} d \boldsymbol{x} d t \\
& -\int_{0}^{T} \int_{D} \boldsymbol{b}^{\epsilon}(\boldsymbol{x}, t) \cdot \boldsymbol{w}(\boldsymbol{x}, t) d \boldsymbol{x} d t .
\end{aligned}
$$

and an integration by parts gives the strong form

$$
\rho \ddot{\boldsymbol{u}}^{\epsilon}(\boldsymbol{x}, t)=\mathcal{L}^{\epsilon}\left(\boldsymbol{u}^{\epsilon}\right)(\boldsymbol{x}, t)+\boldsymbol{b}^{\epsilon}(\boldsymbol{x}, t), \text { for } \boldsymbol{x} \in D .
$$

Here $\mathcal{L}^{\epsilon}\left(\boldsymbol{u}^{\epsilon}\right)$ is the peridynamic force

$$
\mathcal{L}^{\epsilon}\left(\boldsymbol{u}^{\epsilon}\right)=\int_{\mathcal{H}_{\epsilon}(x) \cap D} \boldsymbol{f}^{\epsilon}(\boldsymbol{y}, \boldsymbol{x}) d \boldsymbol{y}
$$

and $\boldsymbol{f}^{\epsilon}(\boldsymbol{x}, \boldsymbol{y})$ is given by

$$
\boldsymbol{f}^{\epsilon}(\boldsymbol{x}, \boldsymbol{y})=2 \partial_{S} \mathcal{W}^{\epsilon}\left(\boldsymbol{y}-\boldsymbol{x}, S\left(\boldsymbol{y}, \boldsymbol{x}, \boldsymbol{u}^{\epsilon}(t)\right)\right) \boldsymbol{e}_{\boldsymbol{y}-\boldsymbol{x}},
$$

where

$$
\partial_{S} \mathcal{W}^{\epsilon}\left(\boldsymbol{y}-\boldsymbol{x}, S\left(\boldsymbol{y}, \boldsymbol{x}, \boldsymbol{u}^{\epsilon}(t)\right)\right)=\frac{1}{\epsilon^{3} \omega_{2}} \frac{J^{\epsilon}(|\boldsymbol{y}-\boldsymbol{x}|)}{|\boldsymbol{y}-\boldsymbol{x}|} \partial_{S} \Psi\left(\sqrt{|\boldsymbol{y}-\boldsymbol{x}|} S\left(\boldsymbol{y}, \boldsymbol{x}, \boldsymbol{u}^{\epsilon}(t)\right)\right) .
$$

The dynamics is complemented with the initial data

$$
\boldsymbol{u}^{\epsilon}(\boldsymbol{x}, 0)=\boldsymbol{u}_{0}(\boldsymbol{x}), \quad \partial_{t} \boldsymbol{u}^{\epsilon}(\boldsymbol{x}, 0)=\boldsymbol{v}_{0}(\boldsymbol{x}) .
$$

Where $\boldsymbol{u}_{0}$ and $\boldsymbol{v}_{0}$ lie in $\dot{L}^{2}\left(D ; \mathbb{R}^{2}\right)$. 
The initial value problem for the nonlocal evolution given by (2.16) and (2.20) or equivalently by $(2.15)$ and $(2.20)$ has a unique solution in $C^{2}([0, T]$; $\left.\dot{L}^{2}\left(D ; \mathbb{R}^{2}\right)\right)$, see Sect.

4. Application of Grönwall's inequality shows that the nonlocal evolution $\boldsymbol{u}^{\epsilon}(\boldsymbol{x}, t)$ is uniformly bounded in the mean square norm over the time interval $0<t<T$,

$$
\max _{0<t<T}\left\{\left\|\boldsymbol{u}^{\epsilon}(\boldsymbol{x}, t)\right\|_{L^{2}\left(D ; \mathbb{R}^{2}\right)}^{2}\right\}<K,
$$

where the upper bound $K$ is independent of $\epsilon$ and depends only on the initial conditions and body force applied up to time $T$, see [24].

\subsection{Initial crack, failure zone and softening zone geometry}

In this section the assumptions on the crack geometry and crack velocity are presented. The initial crack is assumed present at the start of the fracture evolution. It is described by a crack centerline lying on the $x_{2}=0$ axis given by the interval $0 \leq x_{1} \leq \ell(0)$. The initial crack centerline can be written as

$$
C=\left\{0 \leq x_{1} \leq \ell(0), x_{2}=0\right\} .
$$

The initial crack is the set of pairs $\boldsymbol{x}$ and $\boldsymbol{y}$ connected by a line segment that is intersected by the centerline with $|\boldsymbol{y}-\boldsymbol{x}|<\epsilon$ for which the force $\boldsymbol{f}^{\epsilon}(\boldsymbol{x}, \boldsymbol{y})$ acting between them is zero. This set can be identified with the subset of the sample given by the union of center points $\boldsymbol{x}$ of open balls $\mathcal{H}_{\epsilon}(\boldsymbol{x})$ that intersect the crack centerline; this is displayed in Fig. 3. Mathematically the initial crack is defined by

$$
\{\boldsymbol{x} \text { and } \boldsymbol{y} \in D,|\boldsymbol{y}-\boldsymbol{x}|<\epsilon: \boldsymbol{x}+s(\boldsymbol{y}-\boldsymbol{x}) \cap C \neq \emptyset \text {, for some } s \in[0,1]\} .
$$

We continue with a heuristic description of the assumptions on crack structure and follow with their mathematical definitions given by $(2.25),(2.27)$, and (2.31). The failure zone $F Z^{\epsilon}(t)$ represents the crack in the nonlocal model at a given time $t$. This is the set of pairs $\boldsymbol{x}$ and $\boldsymbol{y}$ with $|\boldsymbol{y}-\boldsymbol{x}|<\epsilon$ for which the force $\boldsymbol{f}^{\epsilon}(\boldsymbol{x}, \boldsymbol{y})$ acting between them is zero. In this problem the domain and body force adjacent to the upper and lower boundaries are symmetric with respect to the $x_{2}=0$ axis, see (2.9). The body force is perpendicular to the $x_{2}=0$ axis and points in the $e^{2}$ direction on the top boundary layer and the $-e^{2}$ direction on the bottom boundary layer. Choosing initial conditions appropriately the solution to the initial value problem has its first component $u_{1}^{\epsilon}$ even with respect to the $x_{2}=0$ axis and second component $u_{2}^{\epsilon}$ odd for $t \in[0, T]$. For the time dependent body force chosen here the failure is in tension and is assumed to be confined to a neighborhood of the $x_{2}=0$ axis of width $2 \epsilon$ where strains are largest. The failure zone includes the initial crack and is defined by a centerline lying on the $x_{2}=0$ axis. The failure zone propagates continuously from the initial crack into the interior of the specimen. The failure zone centerline is

$$
C^{\epsilon}(t)=\left\{\ell(0) \leq x_{1} \leq \ell^{\epsilon}(t), x_{2}=0\right\} .
$$


The failure zone $F Z^{\epsilon}$ is assumed to be given by

$$
\begin{aligned}
& F Z^{\epsilon}(t)=\{\boldsymbol{x} \text { and } \boldsymbol{y} \in D,|\boldsymbol{y}-\boldsymbol{x}|<\epsilon: \\
& \left.\quad \boldsymbol{x}+s(\boldsymbol{y}-\boldsymbol{x}) \cap C^{\epsilon}(t) \neq \emptyset, \text { for some } s \in[0,1]\right\} .
\end{aligned}
$$

The centerline is shown in Fig. 4 and the failure zone is the shaded region.

The total traction force on on the layer of thickness $\epsilon$ above the failure zone centerline exerted by the body below the failure zone centerline is null and vice versa. Associated with the failure zone is the softening zone. The softening zone $S Z^{\epsilon}(t)$ is the set of pairs $\boldsymbol{x}$ and $\boldsymbol{y}$ with $|\boldsymbol{y}-\boldsymbol{x}|<\epsilon$ separated by the $x_{2}=0$ axis such that the force $\boldsymbol{f}^{\epsilon}(\boldsymbol{x}, \boldsymbol{y})$ between them is non-increasing with increasing strain. From this it is clear that $F Z^{\epsilon}(t) \subset S Z^{\epsilon}(t)$. Furthermore at the leading edge of the crack one sees force softening between points $\boldsymbol{x}$ and $\boldsymbol{y}$ separated by less than $\epsilon$ on either side of the $x_{2}=0$ axis. As the crack centerline moves forward passing between $\boldsymbol{x}$ and $\boldsymbol{y}$ the force between $\boldsymbol{x}$ and $\boldsymbol{y}$ decreases to zero, see Fig. 4 . That is given $t$ there is a later time $t+\Delta t$ for which $F Z^{\epsilon}(t+\Delta t)=S Z^{\epsilon}(t)$. The process zone where the bonds have softened but not failed, i.e., $\boldsymbol{x}, \boldsymbol{y} \in S Z^{\epsilon}(t) \backslash F Z^{\epsilon}(t)$ is assumed to be of length proportional to $\epsilon$. The softening zone $S Z^{\epsilon}(t)$ is specified through a softening zone centerline. The force between two points $\boldsymbol{x}$ and $\boldsymbol{y}$ separated by the softening zone centerline decreases with time. The centerline is

$$
S^{\epsilon}(t)=\left\{\ell(0) \leq x_{1} \leq \ell^{\epsilon}(t)+C \epsilon, x_{2}=0\right\},
$$

here we assume $C$ is a positive constant. The softening zone is assumed to be given by

$$
\begin{aligned}
& S Z^{\epsilon}(t)=\{\boldsymbol{x} \text { and } \boldsymbol{y} \in D,|\boldsymbol{y}-\boldsymbol{x}| \\
&\left.<\epsilon: \boldsymbol{x}+s(\boldsymbol{y}-\boldsymbol{x}) \cap S^{\epsilon}(t) \neq \emptyset, \text { for some } s \in[0,1]\right\} .
\end{aligned}
$$

We note that the shapes of $F Z^{\epsilon}$ and $S Z^{\epsilon}$ are consequences of their definition similar to the initial crack. In what follows the crack does not propagate all the way through the sample, i.e., $\ell^{\epsilon}(T)<a-\delta$, for every $\epsilon$ where $\delta$ is a small fixed positive constant.

The strain $S\left(\boldsymbol{y}, \boldsymbol{x}, \boldsymbol{u}^{\epsilon}(t)\right)$ is decomposed for $\boldsymbol{x}$ and $\boldsymbol{y}$ in $D$ and $|\boldsymbol{y}-\boldsymbol{x}|<\epsilon$ as

$$
S\left(\boldsymbol{y}, \boldsymbol{x}, \boldsymbol{u}^{\epsilon}(t)\right)=S\left(\boldsymbol{y}, \boldsymbol{x}, \boldsymbol{u}^{\epsilon}(t)\right)^{-}+S\left(\boldsymbol{y}, \boldsymbol{x}, \boldsymbol{u}^{\epsilon}(t)\right)^{+}
$$

where

$$
S\left(\boldsymbol{y}, \boldsymbol{x}, \boldsymbol{u}^{\epsilon}(t)\right)^{-}= \begin{cases}S\left(\boldsymbol{y}, \boldsymbol{x}, \boldsymbol{u}^{\epsilon}(t)\right), & \text { if }\left|S\left(\boldsymbol{y}, \boldsymbol{x}, \boldsymbol{u}^{\epsilon}(t)\right)\right|<S_{c} \\ 0, & \text { otherwise }\end{cases}
$$

and

$$
S\left(\boldsymbol{y}, \boldsymbol{x}, \boldsymbol{u}^{\epsilon}(t)\right)^{+}= \begin{cases}S\left(\boldsymbol{y}, \boldsymbol{x}, \boldsymbol{u}^{\epsilon}(t)\right), & \text { if }\left|S\left(\boldsymbol{y}, \boldsymbol{x}, \boldsymbol{u}^{\epsilon}(t)\right)\right| \geq S_{c} \\ 0, & \text { otherwise }\end{cases}
$$

with

$$
\begin{aligned}
& \left\{\boldsymbol{x} \text { and } \boldsymbol{y} \in D: S\left(\boldsymbol{y}, \boldsymbol{x}, \boldsymbol{u}^{\epsilon}(t)\right)^{+}>0\right\}=\left\{(\boldsymbol{x}, \boldsymbol{y}) \in S Z^{\epsilon}(t)\right\}, \\
& \left\{\boldsymbol{x} \text { and } \boldsymbol{y} \in D: S\left(\boldsymbol{y}, \boldsymbol{x}, \boldsymbol{u}^{\epsilon}(t)\right)^{-}>0\right\}=\left\{(\boldsymbol{x}, \boldsymbol{y}) \notin S Z^{\epsilon}(t)\right\} .
\end{aligned}
$$




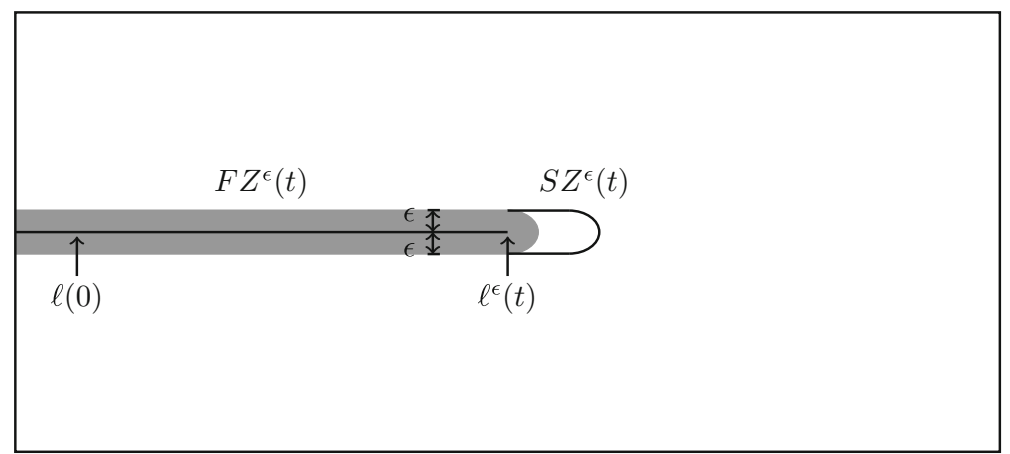

FiguRE 4. The failure zone, failure zone centerline, and softening zone

In the next section we recover the dynamics in the limit of vanishing horizon with failure zone and softening zone given by (2.25) and (2.27). The equations (2.25), (2.27), and (2.31) together with the continuous and monotonic growth of the crack centerline (2.24) constitute the hypothesis on the crack structure for the nonlocal model. For the loading prescribed here (2.25) and (2.27) naturally grow continuously and are a consequence of the symmetry of solution $\boldsymbol{u}^{\epsilon}(\boldsymbol{x}, t)$, this is seen in simulations [22].

\section{Convergence of nonlocal elastodynamics to elastic fields in Linear Elastic Fracture Mechanics}

The crack structure is prescribed by $\ell^{\epsilon}(t)$ of (2.24) together with $(2.25),(2.27)$, and (2.31) and the elastic fields $\boldsymbol{u}^{\epsilon}$ are solutions of (2.16) and (2.20). The crack structure for $\epsilon>0$ is summarized in the following hypothesis:

Hypothesis 3.1. (Crack Structure for $\epsilon>0$.) The moving domain associated with the defect is prescribed by the monotonic and continuous function $\ell^{\epsilon}(t)$ of (2.24), and the failure zone and softening zone are given by (2.25), (2.27), and (2.31).

Given hypothesis 3.1 we now describe the convergence of $\boldsymbol{u}^{\epsilon}$ to $\boldsymbol{u}^{0}$ to see that $\boldsymbol{u}^{0}$ satisfies the boundary value problem for the elastic field of LEFM for a running crack given in [17]. Recall $\ell^{\epsilon}(t)$ is monotone increasing with time and bounded so from Helly's selection theorem we can pass to a subsequence if necessary to assert that $\ell^{\epsilon_{n}}(t) \rightarrow \ell^{0}(t)$ point wise for $t \in[0, T]$, where $\ell^{0}(t)$ is monotone increasing with time and bounded. This delivers the crack motion for the $\epsilon=0$ problem described by the crack

$$
\Gamma_{t}=\left\{\ell(0) \leq x_{1} \leq \ell^{0}(t), x_{2}=0\right\}, t \in[0, T] .
$$

Here $\tau<t$ implies $\Gamma_{\tau} \subset \Gamma_{t}$. The time dependent domain surrounding the crack is defined as $D_{t}=D \backslash \Gamma_{t}$ see Fig. 5 . 
Next we describe the convergence of body force, velocity, and acceleration given by the $\epsilon>0$ initial value problems (2.16) and (2.20) to their $\epsilon=0$ counterparts. The convergence of the elastic displacement field, velocity field and acceleration field are described in terms of suitable Hilbert space topologies. The space of strongly measurable functions $\boldsymbol{w}:[0, T] \rightarrow \dot{L}^{2}\left(D ; \mathbb{R}^{2}\right)$ that are square integrable in time is denoted by $L^{2}\left(0, T ; \dot{L}^{2}\left(D ; \mathbb{R}^{2}\right)\right)$. Additionally we recall the Sobolev space $H^{1}\left(D ; \mathbb{R}^{2}\right)$ with norm

$$
\|\boldsymbol{w}\|_{H^{1}\left(D ; \mathbb{R}^{2}\right)}:=\left(\int_{D}|\boldsymbol{w}|^{2} d \boldsymbol{x}+\int_{D}|\nabla \boldsymbol{w}|^{2} d \boldsymbol{x}\right)^{1 / 2} .
$$

and $\dot{H}^{1}\left(D ; \mathbb{R}^{2}\right)=H^{1}\left(D ; \mathbb{R}^{2}\right) \cap \dot{L}^{2}\left(D ; \mathbb{R}^{2}\right)$. The Hilbert space dual to $\dot{H}^{1}\left(D ; \mathbb{R}^{2}\right)$ is denoted by $\dot{H}^{1}\left(D ; \mathbb{R}^{2}\right)^{\prime}$. The set of functions strongly square integrable in time taking values in $\dot{H}^{1}\left(D ; \mathbb{R}^{2}\right)^{\prime}$ for $0 \leq t \leq T$ is denoted by $L^{2}\left(0, T ; \dot{H}^{1}(D\right.$; $\left.\left.\mathbb{R}^{2}\right)^{\prime}\right)$. These Hilbert spaces are well known and related to the wave equation, see $[13,15]$. For future reference we write the symmetric part of $\nabla \boldsymbol{u}$ as $\mathcal{E} \boldsymbol{w}=$ $\left(\nabla \boldsymbol{w}+\nabla \boldsymbol{w}^{T}\right) / 2$.

The body force given in (2.16) is written as $\boldsymbol{b}^{\epsilon_{n}}(t)$ and we state the following lemma.

Lemma 3.1. There is a positive constant $C$ independent of $\epsilon_{n}$ and $t \in[0, T]$ such that

$$
\left|\left\langle\boldsymbol{b}^{\epsilon_{n}}(t), \boldsymbol{w}\right\rangle\right| \leq C\|\boldsymbol{w}\|_{H^{1}\left(D, \mathbb{R}^{2}\right)}, \text { for all } \epsilon_{n}>0 \text { and } \boldsymbol{w} \in \dot{H}^{1}\left(D, \mathbb{R}^{2}\right),
$$

where $\langle\cdot, \cdot\rangle$ is the duality paring between $\dot{H}^{1}\left(D, \mathbb{R}^{2}\right)$ and its Hilbert space dual $\dot{H}^{1}\left(D, \mathbb{R}^{2}\right)^{\prime}$. In addition there exists $\boldsymbol{b}^{0}(t)$ such that $\boldsymbol{b}^{\epsilon_{n}} \rightarrow \boldsymbol{b}^{0}$ in $L^{2}$ $\left(0, T ; \dot{H}^{1}\left(D ; \mathbb{R}^{2}\right)^{\prime}\right)$ and

$$
\left\langle\boldsymbol{b}^{0}(t), \boldsymbol{w}\right\rangle=\langle\boldsymbol{g}(t), \boldsymbol{w}\rangle:=\int_{\partial D} \boldsymbol{g}(t) \cdot \boldsymbol{w} d \sigma,
$$

for all $\boldsymbol{w} \in \dot{H}^{1}\left(D, \mathbb{R}^{2}\right)$, where $\boldsymbol{g}(t)$ is defined by (2.11) and $\boldsymbol{g} \in H^{-1 / 2}(\partial D)^{2}$.

The traction force (3.4) delivers loading consistent with a mode one crack in the local model given by LEFM. For ease of exposition we defer the proof of Lemma 3.1 as well as proofs of all other theorems introduced here to Sects. 5 and 6 .

Passing to subsequences as necessary we obtain the convergence of the elastic displacement field, velocity field, and acceleration field given by

\section{Lemma 3.2.}

$$
\begin{aligned}
& \boldsymbol{u}^{\epsilon_{n}} \rightarrow \boldsymbol{u}^{0} \text { strong in } C\left([0, T] ; \dot{L}^{2}\left(D ; \mathbb{R}^{2}\right)\right) \\
& \dot{\boldsymbol{u}}^{\epsilon_{n}} \rightarrow \dot{\boldsymbol{u}}^{0} \text { weakly in } L^{2}\left(0, T ; \dot{L}^{2}\left(D ; \mathbb{R}^{2}\right)\right) \\
& \ddot{\boldsymbol{u}}^{\epsilon_{n}} \rightarrow \ddot{\boldsymbol{u}}^{0} \text { weakly in } L^{2}\left(0, T ; \dot{H}^{1}\left(D ; \mathbb{R}^{2}\right)^{\prime}\right) \\
& \ddot{\boldsymbol{u}}^{\epsilon_{n}} \rightarrow \ddot{\boldsymbol{u}}^{0} \text { weakly in } L^{2}\left(0, T ; H^{-1}\left(D ; \mathbb{R}^{2}\right)\right),
\end{aligned}
$$

where $\dot{\boldsymbol{u}}^{0}(t)$ and $\ddot{\boldsymbol{u}}^{0}(t)$ are distributional derivatives in time. 
With the additional caveat that

$$
\sup _{[0, T] \epsilon>0}\left\|\boldsymbol{u}^{\epsilon}(t)\right\|_{L^{\infty}\left(D, \mathbb{R}^{2}\right)}<\infty
$$

the limit evolution $\boldsymbol{u}^{0}(\boldsymbol{x}, t)$ is seen to be an element of the Sobolev space $\dot{H}^{1}\left(D_{t}, \mathbb{R}^{2}\right)$, (with norm $(3.2)$ ), for almost all times in $0<t<T$. This is summarized in the following theorem.

Theorem 3.1. The displacement $\boldsymbol{u}^{0}$ is in the Sobolev space $\dot{H}^{1}\left(D_{t}, \mathbb{R}^{2}\right)$ for a.e. $t \in(0, T)$ and its first component denoted by $u_{1}^{0}\left(x_{1}, x_{2}\right)$ is even with respect to the $x_{2}=0$ axis and the second component of the displacement denoted by $u_{2}^{0}\left(x_{1}, x_{2}\right)$ is odd with respect to the $x_{2}=0$ axis and $u_{2}^{0}\left(x_{1}, 0\right)=0, \mathcal{H}^{1}$ a.e. for $\left\{\ell^{0}(t)<x_{1}<a, x_{2}=0\right\}$.

This theorem is proved in Sect. 5. The assumption (3.6) is used to conclude that $\boldsymbol{u}^{0}$ is in the space of Special of Functions of Bounded Deformation (SBD). This type of assumption is used in passing to SBD limits in quasistatic fracture energies based on discrete and continuous formulations [1] and in SBV for scalar problems in image processing [19]. For dynamic problems it is used in showing $\boldsymbol{u}^{0}(t) \in S B D$ for almost all times $t \in(0, T)$, see [24]. Once it is established that $\boldsymbol{u}^{0}(t)$ belongs to SBD for almost all times, the symmetry of solution and the crack growth hypothesis are used to show $\boldsymbol{u}^{0}(t) \in \dot{H}^{1}\left(D_{t}, \mathbb{R}^{2}\right)$, a.e. $t \in(0, T)$.

The global description of $\ddot{\boldsymbol{u}}^{0}(t)$ can be further specified in terms of suitable Sobolev spaces posed over time dependent domains. For $0 \leq \tau \leq t$, monotonicty implies $\ell(0)=\ell^{0}(0) \leq \ell^{0}(\tau) \leq \ell^{0}(t)$. We choose $0<\beta<\ell(0)$ and introduce $D_{\beta}(t)=D \backslash\left\{\ell(0) \leq x_{1} \leq \ell^{0}(t)-\beta ; x_{2}=0\right\}$. It is evident that $D_{t} \subset D_{\beta}(t)$ and its boundary is denoted by $\partial D_{\beta}(t)$. The subsets of the boundary $\partial D_{\beta}(t)$ bordering the domains $\left\{\boldsymbol{x} \in D_{\beta}(t): \pm x_{2} \geq 0\right\}$ are denoted by $\partial D_{\beta}^{ \pm}(t)$. The layer $L_{\beta}^{+}(t)$ adjacent to $\partial D_{\beta}^{+}(t)$ is defined to be the region inside the solid and dashed contours drawn in Fig. 6 . The dashed contour interior to $D_{\beta}(t)$ is denoted by $\partial L^{+}$and described by the polygonal line connecting the points; $\left(\ell^{0}(t)-\beta, 0\right),\left(\ell^{0}(t)-\beta, b / 10\right),(a-\delta, b / 10),(a-\delta, 0),(a, 0)$, but any polygonal line that avoids the crack tip similar to the one in Fig. 6 will suffice. For $0<t<T$ set

$$
\begin{aligned}
& W^{+}\left(D_{\beta}(t)\right) \\
& \quad=\left\{\boldsymbol{w} \in H^{1}\left(L_{\beta}^{+}(t), \mathbb{R}^{2}\right) \text { and } \gamma \boldsymbol{w}=0 \text { on } \partial L^{+}, \boldsymbol{w} \text { extended by } 0 \text { to } D_{\beta}(t)\right\},
\end{aligned}
$$

here $\gamma$ is the trace operator mapping functions in $H^{1}\left(L_{\beta}^{+}(t), \mathbb{R}^{2}\right)$ to functions defined on the boundary. The Hilbert space dual to $W^{+}\left(D_{\beta}(t)\right)$ is denoted by $W^{+}\left(D_{\beta}(t)\right)^{\prime}$. We introduce the layer $L_{\beta}^{-}(t)$ adjacent to the boundary $\partial D_{\beta}^{-}(t)$ 
and the boundary of the layer internal to $D_{\beta}(t)$ is denoted by $\partial L^{-}$. The analogous space $W^{-}\left(D_{\beta}(t)\right)$ is given by

$$
\begin{aligned}
& W^{-}\left(D_{\beta}(t)\right) \\
& \quad=\left\{\boldsymbol{w} \in H^{1}\left(L_{\beta}^{-}(t), \mathbb{R}^{2}\right) \text { and } \gamma \boldsymbol{w}=0 \text { on } \partial L^{-}, \boldsymbol{w} \text { extended by } 0 \text { to } D_{\beta}(t)\right\},
\end{aligned}
$$

with dual $W^{-}\left(D_{\beta}(t)\right)^{\prime}$.

For any $\tau \in(0, T)$ let $\boldsymbol{u}_{\tau}^{0}$ be the restriction of $\boldsymbol{u}^{0}$ to $\tau<t<T$. Then we have the following theorem.

Theorem 3.2. For all $\tau \in(0, T), \ddot{\boldsymbol{u}}_{\tau}^{0}(\boldsymbol{x}, t)$ belongs to $W^{ \pm}\left(D_{\beta}(\tau)\right)^{\prime}$ for almost all $t \in(\tau, T)$ and

$$
\ddot{\boldsymbol{u}}^{\epsilon_{n}} \rightarrow \ddot{\boldsymbol{u}}_{\tau}^{0} \text { weakly in } L^{2}\left(\tau, T ; W^{ \pm}\left(D_{\beta}(\tau)\right)^{\prime}\right) .
$$

Since $\ddot{\boldsymbol{u}}_{\tau}^{0}$ belongs to $W^{ \pm}\left(D_{\beta}(\tau)\right)^{\prime}$ we introduce the the normal traction $\mathbb{C} \mathcal{E} \boldsymbol{u}^{0} \boldsymbol{n}$ defined on the crack lips for $(\tau, T)$ and $\partial D$ in the generalized sense [28]. In order to describe the generalized traction we introduce trace spaces compatible with the crack geometry. For $t \in[0, t]$ we introduce the weight defined on $\partial D_{\beta}^{ \pm}(t)$ given by

$$
\alpha_{ \pm}\left(x_{1}, x_{2}, \beta\right)= \begin{cases}\min \left\{1, \sqrt{\left(\ell^{0}(t)-\beta-x_{1}\right)}\right\}, & \text { on } x_{2}=0 \\ \min \left\{1, \sqrt{ \pm x_{2}}\right\}, & \text { on } x_{1}=a, \pm x_{2}>0 \\ 1, & \text { otherwise. }\end{cases}
$$

and the trace spaces $H_{00}^{1 / 2}\left(\partial D_{\beta}^{ \pm}(t)\right)^{2}$ given in [27] are defined by all functions $\boldsymbol{w}$ in $H^{1 / 2}\left(\partial D_{\beta}^{ \pm}(t)\right)^{2}$ with

$$
\int_{\partial D_{\beta}^{ \pm}(t)}|\boldsymbol{w}(\boldsymbol{x})|^{2} \alpha_{ \pm}^{-1}(\boldsymbol{x}, \beta) d s<\infty .
$$

The dual to $H_{00}^{1 / 2}\left(\partial D_{\beta}^{ \pm}(t)\right)^{2}$ is $H_{00}^{-1 / 2}\left(\partial D_{\beta}^{ \pm}(t)\right)^{2}$. This type of trace space is employed for problems of mechanical contact in [21], see also [33]. The trace operator $\gamma$ is a continuous linear map from $W^{ \pm}\left(D_{\beta}(t)\right)$ onto $H_{00}^{1 / 2}\left(\partial D_{\beta}^{ \pm}(t)\right)^{2}$, see [27]. Additionally the trace operator $\gamma$ is a continuous linear map from $H^{1}\left(D, \mathbb{R}^{2}\right)$ onto $H^{1 / 2}(\partial D)^{2}$.

In what follows the duality bracket for Hilbert spaces $H$ and their dual $H^{\prime}$ is defined by $\langle\cdot, \cdot\rangle$, where the first argument is an element of $H^{\prime}$ and the second an element of $H$. The generalized traction $\mathbb{C} \mathcal{E} \boldsymbol{u}^{0} \boldsymbol{n}$ on $\partial D$ is introduced as an element of $H^{-1 / 2}(\partial D)^{2}$. For this case we have suitable integration by parts formulas given by the following two lemmas.

Lemma 3.3. Given that $\ddot{\boldsymbol{u}}^{0}$ belongs to $\dot{H}^{1}\left(D ; \mathbb{R}^{2}\right)^{\prime}$ and $H^{-1}\left(D ; \mathbb{R}^{2}\right)$, and for $\boldsymbol{w} \in \dot{H}^{1}\left(D ; \mathbb{R}^{2}\right)$, the map $\boldsymbol{w} \rightarrow \int_{D} \mathbb{C} \mathcal{E} \boldsymbol{u}^{0}: \mathcal{E} \boldsymbol{w} d \boldsymbol{x}$ belongs to $\dot{H}^{1}\left(D ; \mathbb{R}^{2}\right)^{\prime}$ 


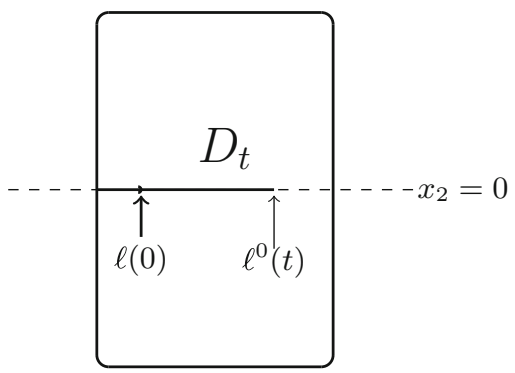

Figure 5. Crack corresponding to $\epsilon=0$ limit

then the generalized traction $\mathbb{C} \mathcal{E} \boldsymbol{u}^{0} \boldsymbol{n}$ is uniquely defined as an element of $H^{-1 / 2}(\partial D)^{2}$ on the boundary $\partial D$ is given by

$$
\left\langle\mathbb{C} \mathcal{E} \boldsymbol{u}^{0} \boldsymbol{n}, \gamma \boldsymbol{w}\right\rangle=\int_{D} \mathbb{C} \mathcal{E} \boldsymbol{u}^{0}: \mathcal{E} \boldsymbol{w} d \boldsymbol{x}+\rho\left\langle\ddot{\boldsymbol{u}}^{0}, \boldsymbol{w}\right\rangle,
$$

for all test functions $\boldsymbol{w}$ in $\dot{H}^{1}\left(D, \mathbb{R}^{2}\right)$ is uniquely defined.

Lemma 3.4. Since $\ddot{\boldsymbol{u}}_{\tau}^{0}(t)$ belongs to $W^{ \pm}\left(D_{\beta}(\tau)\right)^{\prime}$ for a.e., $t \in(\tau, T)$ and $\boldsymbol{u}^{0}(t)$ is in $\dot{H}^{1}\left(D_{t} ; \mathbb{R}^{2}\right)$ the generalized tractions $\mathbb{C} \mathcal{E} \boldsymbol{u}^{0}(t) \boldsymbol{n}^{ \pm}$are uniquely defined as elements of $H_{00}^{-1 / 2}\left(\partial D_{\beta}^{ \pm}(\tau)\right)^{2}$ on the upper and lower sides of the crack $\Gamma_{t}$ by

$$
\left\langle\mathbb{C} \mathcal{E} \boldsymbol{u}^{0}(t) \boldsymbol{n}^{ \pm}, \gamma \boldsymbol{w}\right\rangle=\int_{L_{\beta}^{ \pm}(\tau)} \mathbb{C} \mathcal{E} \boldsymbol{u}^{0}(t): \mathcal{E} \boldsymbol{w} d \boldsymbol{x}+\rho\left\langle\ddot{\boldsymbol{u}}_{\tau}^{0}(t), \boldsymbol{w}\right\rangle,
$$

for all test functions $\boldsymbol{w}$ in $W^{ \pm}\left(D_{\beta}(\tau)\right)$ and a.e., $t \in(\tau, T)$.

Lemmas 3.3 and 3.4 are proved in Sect. 6 .

The global dynamics for $\boldsymbol{u}^{0}(\boldsymbol{x}, t)$ is given by the following theorem.

Theorem 3.3. The limit displacement field $\boldsymbol{u}^{0}$ satisfies

$$
\rho \ddot{\boldsymbol{u}}^{0}=\operatorname{div}\left(\mathbb{C} \mathcal{E} \boldsymbol{u}^{0}\right)
$$

as elements of $H^{-1}\left(D, \mathbb{R}^{2}\right)$, for a.e., $t \in(0, T)$ and

$$
\mathbb{C} \mathcal{E} \boldsymbol{u}^{0} \boldsymbol{n}=\boldsymbol{g} \text { on } \partial D
$$

where the traction $\boldsymbol{g}$ is given by (2.11) and equality holds as elements of $H^{-1 / 2}$ $(\partial D)^{2}$ for a.e., $t \in(0, T)$. Moreover there is zero traction on the upper and lower sides of the crack $\Gamma_{t}, t \in(0, T)$, this is given by

$$
\mathbb{C} \mathcal{E} \boldsymbol{u}^{0}(t) \boldsymbol{n}^{ \pm}=0, \text { for } \quad\left\{\ell(0)<x_{1} \leq \ell^{0}(\tau)-\beta ; x_{2}=0\right\}
$$

as elements of $H_{00}^{-1 / 2}\left(\partial D_{\beta}^{ \pm}(\tau)\right)^{2}$ for a.e., $t \in(\tau, T)$, for all $\beta \in\left(0, \ell^{0}(\tau)-\ell(0)\right)$.

Here the normal tractions (3.15) and (3.16) are defined in the generalized sense (3.12), (3.13) respectively. To summarize Theorem 3.3 delivers the global description of the displacement fields inside the cracking body. Together they deliver the elastodynamic equations and homogeneous traction boundary conditions on the crack faces given in LEFM [3,17,32], and [38]. 
We remark here that in Theorem 3.2 we restrict to times larger than a fixed positive time $\tau$ to show the normal traction on the crack faces are zero on any open subset set of the crack away from the crack tip. The case $\tau=t$ is not considered because of the singularity in the elastic field at the crack tip.

The field $\boldsymbol{u}^{0}(t, \boldsymbol{x})$ is seen to be a weak solution of the wave equation on $D_{t}$ for $t \in[0, T]$. We begin with the definition of weak solution of the wave equation on time dependent domains introduced in [12]. Neumann boundary conditions are considered and set $V_{t}=\dot{H}^{1}\left(D_{t}, \mathbb{R}^{2}\right), V_{t}^{*}=\dot{H}^{1}\left(D_{t}, \mathbb{R}^{2}\right)^{\prime}$ for $t \in[0, T]$, and $H=\dot{L}^{2}\left(D, \mathbb{R}^{2}\right)$. Recall $\Gamma_{s} \subset \Gamma_{t}$ when $0 \leq s \leq t \leq T$ and $\mathcal{H}^{1}\left(\Gamma_{T}\right)<a-\ell(0)$.

Definition 3.1. [12] $\mathcal{V}$ is the space of functions $\boldsymbol{v} \in L^{2}\left(0, T ; V_{T}\right) \cap H^{1}(0, T ; H)$ such that $\boldsymbol{v}(t) \in V_{t}$ for a.e. $t \in(0, T)$. It is a Hilbert space with scalar product given by

$$
(\boldsymbol{u}, \boldsymbol{v})_{\mathcal{V}}=(\boldsymbol{u}, \boldsymbol{v})_{L^{2}\left(0, T ; V_{T}\right)}+(\dot{\boldsymbol{u}}, \dot{\boldsymbol{v}})_{L^{2}(0, T ; H)}
$$

where $\dot{\boldsymbol{u}}$ and $\dot{\boldsymbol{v}}$ denote distributional derivatives with respect to $t$.

Definition 3.2. [12] Given $\boldsymbol{g}(t)$ defined by (2.11) the displacement $\boldsymbol{u}$ is said to be a weak solution of the wave equation

$$
\left\{\begin{array}{l}
\rho \ddot{\boldsymbol{u}}(t)+\operatorname{div}(\mathbb{C} \mathcal{E} \boldsymbol{u}(t))=0 \\
\mathbb{C} \mathcal{E} \boldsymbol{u}(t) \boldsymbol{n}=\boldsymbol{g}(t), \text { on } \partial D \\
\boldsymbol{u}(t) \in V_{t}
\end{array}\right.
$$

on the time interval $[0, T]$ if $\boldsymbol{u} \in \mathcal{V}$ and

$$
\begin{aligned}
& -\int_{0}^{T} \rho \int_{D} \dot{\boldsymbol{u}}(t) \cdot \dot{\varphi}(t) d \boldsymbol{x} d t+\int_{0}^{T} \int_{D} \mathbb{C} \mathcal{E} \boldsymbol{u}(t): \mathcal{E} \varphi(t) d \boldsymbol{x} d t \\
& =\int_{0}^{T} \int_{\partial D} \boldsymbol{g}(t) \cdot \varphi(t) d \sigma d t
\end{aligned}
$$

for every $\varphi \in \mathcal{V}$ with $\varphi(T)=\varphi(0)=0$.

Theorem 3.4. If the crack tip $\ell^{0}(t)$ is continuous and strictly increasing for $t \in[0, T]$ then the limit displacement $\boldsymbol{u}^{0}$ is a weak solution of the wave equation on $D_{t}$ for $t \in[0, T]$ given by Definition 3.2 .

Theorem 3.4 establishes the link between the nonlocal theory and the theory of the wave equation on time dependent domains [12]. Here the choice of test functions delivers a variational description of vanishing normal traction for the solution of the weak formulation.

\section{Existence and uniqueness of nonlocal elastodynamics}

We assert the existence and uniqueness for a solution $\boldsymbol{u}^{\epsilon}(\boldsymbol{x}, t)$ of the nonlocal evolution with the balance of momentum given in strong form (2.16). 


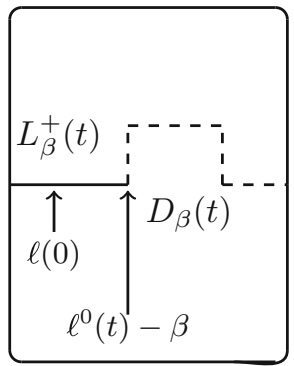

Figure 6 . Domain $L_{\beta}^{+}(t)$ adjacent to $\partial D_{\beta}^{+}(t)$. The boundary of $L_{\beta}^{+}(t)$ interior to $D_{\beta}(t)$ is denoted by the dashed line

Theorem 4.1. Existence and uniqueness of the nonlocal evolution. The initial value problem given by (2.16) and (2.20) has a unique solution $\boldsymbol{u}(\boldsymbol{x}, t)$ such that for every $t \in[0, T], \boldsymbol{u}$ takes values in $\dot{L}^{2}\left(D ; \mathbb{R}^{2}\right)$ and belongs to the space $C^{2}\left([0, T] ; \dot{L}^{2}\left(D ; \mathbb{R}^{2}\right)\right)$.

The proof of this proposition follows from the Lipschitz continuity of $\mathcal{L}^{\epsilon}\left(\boldsymbol{u}^{\epsilon}\right)(\boldsymbol{x}, t)+\boldsymbol{b}(\boldsymbol{x}, t)$ as a function of $\boldsymbol{u}^{\epsilon}$ with respect to the $L^{2}\left(D ; \mathbb{R}^{2}\right)$ norm and the Banach fixed point theorem, see e.g. [25]. It is pointed out that $S Z^{\epsilon}$ describes an unstable phase of the material however because the peridynamic force is a uniformly Lipschitz function on $\dot{L}^{2}\left(D ; \mathbb{R}^{2}\right)$ the model can be viewed as an ODE for vectors in $\dot{L}^{2}\left(D ; \mathbb{R}^{2}\right)$ and is well posed.

\section{Symmetry of the limiting elastic displacement field}

In this section Theorem 3.1 is established. We begin with the caveat (3.6) to conclude that the limit evolution $\boldsymbol{u}^{0}(\boldsymbol{x}, t)$ belongs to $S B D^{2}(D)$ for almost all times $t \in(0, T),[24]$. We then apply Hypothesis 3.1 and Lemma 5.1 to show that the jump set of $\boldsymbol{u}^{0}(t)$ lies inside the crack $\Gamma_{t}$ and then conclude that $\boldsymbol{u}^{0}(t) \in \dot{H}^{1}\left(D_{t}, \mathbb{R}^{2}\right)$ for a.e. $t \in(0, T)$.

For completeness we recall the definitions of $S B D(D)$ and $S B D^{2}(D)$. Functions $\boldsymbol{u} \in S B D(D)$ belong to $L^{1}\left(D ; \mathbb{R}^{2}\right)$ and are approximately continuous, i.e., have Lebesgue limits for almost every $\boldsymbol{x} \in D$ given by

$$
\lim _{\epsilon \searrow 0} \frac{1}{\omega_{2} \epsilon^{2}} \int_{\mathcal{H}_{\epsilon}(\boldsymbol{x})}|\boldsymbol{u}(\boldsymbol{y})-\boldsymbol{u}(\boldsymbol{x})| d \boldsymbol{y}=0,
$$

where $\mathcal{H}_{\epsilon}(\boldsymbol{x})$ is the ball of radius $\epsilon$ centered at $\boldsymbol{x}$ and $\omega_{2} \epsilon^{2}$ is its area given in terms of the area of the unit disk $\omega_{2}$ times $\epsilon^{2}$. The set of points in $D$ which are not points of approximate continuity is denoted by $S_{\boldsymbol{u}}$. A subset of these points are given by the jump set $\mathcal{J}_{u}$. The jump set is defined to be the set of points of discontinuity which have two different one sided Lebesgue limits. One sided Lebesgue limits of $\boldsymbol{u}$ with respect to a direction $\nu_{\boldsymbol{u}(\boldsymbol{x})}$ are denoted 
by $\boldsymbol{u}^{-}(\boldsymbol{x}), \boldsymbol{u}^{+}(\boldsymbol{x})$ and are given by

$$
\begin{aligned}
& \lim _{\epsilon \searrow 0} \frac{1}{\epsilon^{2} \omega_{2}} \int_{\mathcal{H}_{\epsilon}^{-}(x)}\left|\boldsymbol{u}(\boldsymbol{y})-\boldsymbol{u}^{-}(\boldsymbol{x})\right| d \boldsymbol{y}=0, \\
& \lim _{\epsilon \searrow 0} \frac{1}{\epsilon^{2} \omega_{2}} \int_{\mathcal{H}_{\epsilon}^{+}(\boldsymbol{x})}\left|\boldsymbol{u}(\boldsymbol{y})-\boldsymbol{u}^{+}(\boldsymbol{x})\right| d \boldsymbol{y}=0,
\end{aligned}
$$

where $\mathcal{H}_{\epsilon}^{-}(\boldsymbol{x})$ and $\mathcal{H}_{\epsilon}^{+}(\boldsymbol{x})$ are given by the intersection of $\mathcal{H}_{\epsilon}(\boldsymbol{x})$ with the half spaces $(\boldsymbol{y}-\boldsymbol{x}) \cdot \nu_{\boldsymbol{u}(\boldsymbol{x})}<0$ and $(\boldsymbol{y}-\boldsymbol{x}) \cdot \nu_{\boldsymbol{u}(\boldsymbol{x})}>0$ respectively. $S B D(D)$ functions have jump sets $\mathcal{J}_{\boldsymbol{u}}$, that are countably rectifiable. Hence they are described by a countable number of components $K_{1}, K_{2}, \ldots$, contained within smooth manifolds, with the exception of a set $K_{0}$ that has zero 1 dimensional Hausdorff measure [2]. The one dimensional Hausdorff measure of $\mathcal{J}_{u}$ agrees with the one dimensional Lebesgue measure and $\mathcal{H}^{1}\left(\mathcal{J}_{\boldsymbol{u}}\right)=\sum_{i} \mathcal{H}^{1}\left(K_{i}\right)$. The strain of a displacement $\boldsymbol{u}$ belonging to $S B D(D)$, written as $\mathcal{E}_{i j} \boldsymbol{u}^{0}(t)=\left(\partial_{x_{i}} \boldsymbol{u}_{j}^{0}+\right.$ $\left.\partial_{x_{j}} \boldsymbol{u}_{i}^{0}\right) / 2$, is a generalization of the classic local strain tensor and is related to the nonlocal strain $S\left(\boldsymbol{y}, \boldsymbol{x}, \boldsymbol{u}^{0}\right)$ by

$$
\lim _{\epsilon \rightarrow 0} \frac{1}{\epsilon^{2} \omega_{2}} \int_{\mathcal{H}_{\epsilon}(\boldsymbol{x})}\left|S\left(\boldsymbol{y}, \boldsymbol{x}, \boldsymbol{u}^{0}\right)-\mathcal{E} \boldsymbol{u}^{0}(\boldsymbol{x}) \boldsymbol{e} \cdot \boldsymbol{e}\right| d \boldsymbol{y}=0
$$

for almost every $\boldsymbol{x}$ in $D$ with respect to 2-dimensional Lebesgue measure $\mathcal{L}^{2}$. The symmetric part of the distributional derivative of $\boldsymbol{u}, E \boldsymbol{u}=1 / 2\left(\nabla \boldsymbol{u}+\nabla \boldsymbol{u}^{T}\right)$ for $S B D(D)$ functions is a $2 \times 2$ matrix valued Radon measure with absolutely continuous part with respect to two dimensional Lebesgue measure described by the density $\mathcal{E} \boldsymbol{u}$ and singular part described by the jump set [2] and

$$
\langle E u, \Phi\rangle=\int_{D} \sum_{i, j=1}^{d} \mathcal{E} u_{i j} \Phi_{i j} d \boldsymbol{x}+\int_{\mathcal{J}_{u}} \sum_{i, j=1}^{d}\left(\boldsymbol{u}_{i}^{+}-\boldsymbol{u}_{i}^{-}\right) \boldsymbol{n}_{j} \Phi_{i j} d \mathcal{H}^{1}
$$

for every continuous, symmetric matrix valued test function $\Phi$. In the sequel we will write $[\boldsymbol{u}]=\boldsymbol{u}^{+}-\boldsymbol{u}^{-}$.

The limit dynamics and LEFM energy are expressed in terms of elastic moduli $\lambda$ and $\mu$ and fracture toughness $\mathcal{G}$. These are calculated directly from the nonlocal potential (2.3). Here we have taken the choice $\Psi(r)=h\left(r^{2}\right)$ and the elastic moduli are given by

$$
\mu=\lambda=M \frac{1}{4} h^{\prime}(0)
$$

where the constant $M=\int_{0}^{1} r^{2} J(r) d r$. The elasticity tensor is given by

$$
\mathbb{C}_{i j k l}=2 \mu\left(\frac{\delta_{i k} \delta_{j l}+\delta_{i l} \delta_{j k}}{2}\right)+\lambda \delta_{i j} \delta_{k l}
$$

and

$$
\mathcal{G}_{c}=\frac{4}{\pi} \int_{0}^{1} h\left(S_{+}\right) r^{2} J(r) d r
$$


The limit evolution $\boldsymbol{u}^{0}$ has bounded Griffith surface energy and elastic energy, [24] given by

$$
\int_{D} \mu\left|\mathcal{E} \boldsymbol{u}^{0}(t)\right|^{2}+\frac{\lambda}{2}\left|\operatorname{div} \boldsymbol{u}^{0}(t)\right|^{2} d \boldsymbol{x}+\mathcal{G H}^{1}\left(\mathcal{J}_{\boldsymbol{u}^{0}(t)}\right) \leq C,
$$

for $0 \leq t \leq T$, where $\mathcal{J}_{\boldsymbol{u}^{0}(t)}$ denotes the evolving jump set inside the domain $D$, across which the displacement $\boldsymbol{u}^{0}$ has a jump discontinuity and $\mathcal{H}^{1}$ is one dimensional Hausdorff measure, see [24]. Because $\boldsymbol{u}^{0}$ has bounded energy (5.8) we see that $\boldsymbol{u}^{0}$ also belongs to $S B D^{2}(D)$. Here $S B D^{2}(D)$ is the set of $S B D(D)$ functions with square integrable strain $\mathcal{E} \boldsymbol{u}$ and jump set with bounded $\mathcal{H}^{1}$ measure. It has been recently shown in [8] that for $\boldsymbol{u} \in S B D^{2}(D)$ the following holds

$$
\mathcal{H}^{1}\left(S_{\boldsymbol{u}} \backslash \mathcal{J}_{\boldsymbol{u}}\right)=0
$$

It is remarked that the equality $\lambda=\mu$ appearing in (5.5) is a consequence of the central force nature of the nonlocal interaction mediated by (2.3). While non-central force potentials can deliver a larger class of energy-volume-shape change relations [36] a central force potential is been chosen to illustrate the ideas.

The symmetry of $\boldsymbol{u}^{\epsilon}$ described in Sect. 2.2 together with the crack growth hypothesis are now used to show $\boldsymbol{u}^{0}(t) \in \dot{H}^{1}\left(D_{t}, \mathbb{R}^{2}\right)$, a.e. $t \in(0, T)$. The first lemma that we prove is used to show that the jump set of $\boldsymbol{u}^{0}(t) \in S B D^{2}(D)$ is a subset of $\Gamma_{t}$, a.e. $t \in(0, T)$ so that $\boldsymbol{u}^{0}(t)$ belongs to $\dot{H}^{1}\left(D_{t}, \mathbb{R}^{2}\right)$, a.e. $t \in(0, T)$.

\section{Lemma 5.1.}

$$
\begin{aligned}
& \lim _{\epsilon_{n} \rightarrow 0} \frac{1}{\epsilon_{n}^{2} \omega_{2}} \int_{D} \int_{\mathcal{H}_{\epsilon_{n}}(\boldsymbol{x}) \cap D} \frac{|\boldsymbol{y}-\boldsymbol{x}|}{\epsilon_{n}} J^{\epsilon_{n}}(|\boldsymbol{y}-\boldsymbol{x}|) S\left(\boldsymbol{y}, \boldsymbol{x}, \boldsymbol{u}^{\epsilon_{n}}(t)\right)^{-} d \boldsymbol{y} \varphi(\boldsymbol{x}) d \boldsymbol{x} \\
& \quad=\int_{D} \operatorname{div} \boldsymbol{u}^{0}(\boldsymbol{x}, t) \varphi(\boldsymbol{x}) d \boldsymbol{x} \\
& \lim _{\epsilon_{n} \rightarrow 0} \frac{1}{\epsilon_{n}^{2} \omega_{2}} \int_{S Z^{\epsilon_{n}}} \int_{\mathcal{H}_{\epsilon_{n}}(\boldsymbol{x}) \cap D} \frac{|\boldsymbol{y}-\boldsymbol{x}|}{\epsilon_{n}} J^{\epsilon_{n}}(|\boldsymbol{y}-\boldsymbol{x}|) S\left(\boldsymbol{y}, \boldsymbol{x}, \boldsymbol{u}^{\epsilon_{n}}(t)\right)^{+} d \boldsymbol{y} \varphi(\boldsymbol{x}) d \boldsymbol{x} \\
& =C \int_{\mathcal{J}_{u^{0}(t)}}\left[\boldsymbol{u}^{0}(\boldsymbol{x}, t)\right] \cdot \boldsymbol{n} \varphi(\boldsymbol{x}) d \mathcal{H}^{1}(\boldsymbol{x})
\end{aligned}
$$

for all scalar test functions $\varphi$ that are differentiable with support in D. Here $\left[\boldsymbol{u}^{0}(\boldsymbol{x}, t)\right]$ denotes the jump in displacement across $\mathcal{J}_{\boldsymbol{u}^{0}(t)}$ and $\boldsymbol{n}$ is the unit normal to $\mathcal{J}_{\boldsymbol{u}^{0}(t)}$ and points in the vertical direction $\boldsymbol{e}^{2}$, and $C=\omega_{2} \int_{0}^{1} r^{2} d r$.

Proof of Lemma 5.1. It is convenient to make the change of variables $\boldsymbol{y}=$ $\boldsymbol{x}+\epsilon \xi$ where $\xi$ belongs to the unit disk at the origin $\mathcal{H}_{1}(0)=\{|\xi|<1\}$ and $e=\xi /|\xi|$. The strain is written

$$
\begin{aligned}
\frac{\boldsymbol{u}^{\epsilon}(\boldsymbol{x}+\epsilon \xi)-\boldsymbol{u}^{\epsilon}(\boldsymbol{x})}{\epsilon|\xi|} & :=D_{\boldsymbol{e}}^{\epsilon|\xi|} \boldsymbol{u}^{\epsilon}, \text { and } \\
S\left(\boldsymbol{y}, \boldsymbol{x}, \boldsymbol{u}^{\epsilon}(t)\right) & =D_{\boldsymbol{e}}^{\epsilon|\xi|} \boldsymbol{u}^{\epsilon} \cdot \boldsymbol{e}
\end{aligned}
$$


and for infinitely differentiable scalar valued functions $\varphi$ and vector valued functions $\boldsymbol{w}$ bounded and continuous on $D$ we have

$$
\lim _{\epsilon \rightarrow 0} D_{-e}^{\epsilon|\xi|} \varphi=-\nabla \varphi \cdot e
$$

and

$$
\lim _{\epsilon \rightarrow 0} D_{e}^{\epsilon|\xi|} \boldsymbol{w} \cdot \boldsymbol{e}=\mathcal{E} \boldsymbol{w} e \cdot \boldsymbol{e}
$$

where the convergence is uniform in $D$. We recall $S\left(\boldsymbol{y}, \boldsymbol{x}, \boldsymbol{u}^{\epsilon}(t)\right)^{ \pm}$defined by $(2.29),(2.30)$ and write in terms of the new variables $\xi$ and $\boldsymbol{e}=\xi /|\xi|$ to obtain

$$
\left(D_{\boldsymbol{e}}^{\epsilon|\xi|} \boldsymbol{u}^{\epsilon}(t) \cdot \boldsymbol{e}\right)^{-}= \begin{cases}D_{\boldsymbol{e}}^{\epsilon|\xi|} \boldsymbol{u}^{\epsilon}(t) \cdot \boldsymbol{e}, & \text { if }\left|D_{e}^{\epsilon|\xi|} \boldsymbol{u}^{\epsilon}(t) \cdot \boldsymbol{e}\right|<S_{c}=\frac{r^{c}}{\sqrt{\epsilon_{n}|\xi|}} \\ 0, & \text { otherwise }\end{cases}
$$

and

$$
\left(D_{\boldsymbol{e}}^{\epsilon|\xi|} \boldsymbol{u}^{\epsilon}(t) \cdot \boldsymbol{e}\right)^{+}= \begin{cases}D_{\boldsymbol{e}}^{\epsilon|\xi|} \boldsymbol{u}^{\epsilon}(t) \cdot \boldsymbol{e}, & \text { if }\left|D_{e}^{\epsilon|\xi|} \boldsymbol{u}^{\epsilon}(t) \cdot \boldsymbol{e}\right| \geq S_{c}=\frac{r^{c}}{\sqrt{\epsilon_{n}|\xi|}} \\ 0, & \text { otherwise }\end{cases}
$$

with $D_{e}^{\epsilon|\xi|} \boldsymbol{u}^{\epsilon}(t) \cdot \boldsymbol{e}=\left(D_{\boldsymbol{e}}^{\epsilon|\xi|} \boldsymbol{u}^{\epsilon}(t) \cdot \boldsymbol{e}\right)^{-}+\left(D_{\boldsymbol{e}}^{\epsilon|\xi|} \boldsymbol{u}^{\epsilon}(t) \cdot \boldsymbol{e}\right)^{+}$. We extend $\left(D_{\boldsymbol{e}}^{\epsilon|\xi|} \boldsymbol{u}^{\epsilon} \cdot \boldsymbol{e}\right)^{-}$ by zero when $\boldsymbol{x} \in D$ and $\boldsymbol{x}+\epsilon \xi \notin D$ and

$$
\begin{aligned}
& \frac{1}{\epsilon_{n}^{2} \omega_{2}} \int_{D} \int_{\mathcal{H}_{\epsilon_{n}}(\boldsymbol{x}) \cap D} \frac{|\boldsymbol{y}-\boldsymbol{x}|}{\epsilon_{n}} J^{\epsilon_{n}}(|\boldsymbol{y}-\boldsymbol{x}|)\left|S\left(\boldsymbol{y}, \boldsymbol{x}, \boldsymbol{u}^{\epsilon_{n}}(t)\right)^{-}\right|^{2} d \boldsymbol{y} d \boldsymbol{x} \\
& =\int_{D \times \mathcal{H}_{1}(0)}|\xi| J(|\xi|)\left|\left(D_{\boldsymbol{e}}^{\epsilon_{n}|\xi|} \boldsymbol{u}^{\epsilon_{n}} \cdot \boldsymbol{e}\right)^{-}\right|^{2} d \xi d \boldsymbol{x} .
\end{aligned}
$$

Then as in inequality (6.73) of [24] we have that

$$
\int_{D \times \mathcal{H}_{1}(0)}|\xi| J(|\xi|)\left|\left(D_{\boldsymbol{e}}^{\epsilon_{n}|\xi|} \boldsymbol{u}^{\epsilon_{n}} \cdot \boldsymbol{e}\right)^{-}\right|^{2} d \xi d \boldsymbol{x}<C
$$

for all $\epsilon_{n}>0$. From this we can conclude there exists a function $g(\boldsymbol{x}, \xi)$ such that a subsequence

$$
\left(D_{\boldsymbol{e}}^{\epsilon_{n}|\xi|} \boldsymbol{u}^{\epsilon_{n}} \cdot \boldsymbol{e}\right)^{-} \rightarrow g(\boldsymbol{x}, \xi)
$$

converges weakly in $L^{2}\left(D \times \mathcal{H}_{1}(0), \mathbb{R}\right)$ where the $L^{2}$ norm and inner product are with respect to the weighted measure $|\xi| J(|\xi|) d \xi d \boldsymbol{x}$. Now for any positive number $\eta$ and any subset $D^{\prime}$ compactly contained in $D_{t}$ we can argue as in ([24] proof of lemma 6.6) that $g(\boldsymbol{x}, \xi)=\mathcal{E} \boldsymbol{u}^{0} \boldsymbol{e} \cdot \boldsymbol{e}$ for all points in $D^{\prime}$ with $\operatorname{dist}\left(D^{\prime}, \partial D_{t}\right)>\eta$. Since $D^{\prime}$ and $\eta$ is arbitrary we get that

$$
g(\boldsymbol{x}, \xi)=\mathcal{E} \boldsymbol{u}^{0} e \cdot \boldsymbol{e}
$$


almost everywhere in $D$. Additionally for any smooth scalar test function $\varphi(\boldsymbol{x})$ with compact support in $D$ a straight forward computation gives

$$
\begin{aligned}
\lim _{\epsilon_{n} \rightarrow 0} \int_{D \times \mathcal{H}_{1}(0)}|\xi| J(|\xi|)\left(D_{e}^{\epsilon_{n}|\xi|} \boldsymbol{u}^{\epsilon_{n}} \cdot \boldsymbol{e}\right)^{-} d \xi \varphi(\boldsymbol{x}) d \boldsymbol{x} \\
\quad=\int_{D \times \mathcal{H}_{1}(0)}|\xi| J(|\xi|) g(\boldsymbol{x}, \xi) d \xi \varphi(\boldsymbol{x}) d \boldsymbol{x} \\
\quad=\int_{D \times \mathcal{H}_{1}(0)}|\xi| J(|\xi|) \mathcal{E} \boldsymbol{u}^{0}(\boldsymbol{x}) \boldsymbol{e} \cdot \boldsymbol{e} d \xi \varphi(\boldsymbol{x}) d \boldsymbol{x} \\
=C \int_{D} \operatorname{div}^{0}(\boldsymbol{x}) \varphi(\boldsymbol{x}) d \boldsymbol{x},
\end{aligned}
$$

Here $C=\omega_{2} \int_{0}^{1} r^{2} J(r) d r$ and we have used

$$
\frac{1}{\omega_{2}} \int_{\mathcal{H}_{1}(0)}|\xi| J(|\xi|) \boldsymbol{e}_{i} e_{j} d \xi=\delta_{i j} \int_{0}^{1} r^{2} J(r) d r
$$

On the other hand for any smooth test function $\varphi$ with compact support in $D$ we can integrate by parts and use (5.13) to write

$$
\begin{aligned}
\lim _{\epsilon_{n} \rightarrow 0} & \int_{D \times \mathcal{H}_{1}(0)}|\xi| J(|\xi|) D_{\boldsymbol{e}}^{\epsilon_{n}|\xi|} \boldsymbol{u}^{\epsilon_{n}} \cdot \boldsymbol{e} \varphi(\boldsymbol{x}) d \xi d \boldsymbol{x} \\
& =\lim _{\epsilon_{n} \rightarrow 0} \int_{D \times \mathcal{H}_{1}(0)}|\xi| J(|\xi|) D_{-\boldsymbol{e}}^{\epsilon_{n}|\xi|} \varphi(\boldsymbol{x}) \boldsymbol{u}^{\epsilon_{n}} \cdot \boldsymbol{e}, d \xi d \boldsymbol{x} \\
& =-\int_{D \times \mathcal{H}_{1}(0)}|\xi| J(|\xi|) \boldsymbol{u}^{0} \cdot \boldsymbol{e} \nabla \varphi(\boldsymbol{x}) \cdot \boldsymbol{e} d \xi d \boldsymbol{x} \\
& =-C \int_{D} \boldsymbol{u}^{0} \cdot \nabla \varphi(\boldsymbol{x}) d \boldsymbol{x} \\
& =C \int_{D} \operatorname{tr} E \boldsymbol{u}^{0} \varphi(\boldsymbol{x}) d \boldsymbol{x},
\end{aligned}
$$

where $E \boldsymbol{u}^{0}$ is the strain of the $S B D^{2}$ limit displacement $\boldsymbol{u}^{0}$. Now since $\boldsymbol{u}^{0}$ is in $S B D$ its weak derivative is given by (5.4) and it follows on choosing $\Phi_{i j}=\delta_{i j} \varphi$ that

$$
\int_{D} \operatorname{tr} E \boldsymbol{u}^{0} \varphi d \boldsymbol{x}=\int_{D} \operatorname{div} \boldsymbol{u}^{0} \varphi d \boldsymbol{x}+\int_{\mathcal{J}_{u^{0}(t)}}\left[\boldsymbol{u}^{0}\right] \cdot \boldsymbol{n} \varphi d \mathcal{H}^{1}(\boldsymbol{x})
$$

and

$$
\begin{aligned}
& \int_{D \times \mathcal{H}_{1}(0)}|\xi| J(|\xi|) D_{\boldsymbol{e}}^{\epsilon_{n}|\xi|} \boldsymbol{u}^{\epsilon_{n}} \cdot \boldsymbol{e} d \xi \varphi(\boldsymbol{x}) d \boldsymbol{x} \\
& =\int_{D \times \mathcal{H}_{1}(0)}|\xi| J(|\xi|)\left(D_{\boldsymbol{e}}^{\epsilon_{n}|\xi|} \boldsymbol{u}^{\epsilon_{n}} \cdot \boldsymbol{e}\right)^{-} d \xi \varphi(\boldsymbol{x}) d \boldsymbol{x} \\
& \quad+\int_{D \times \mathcal{H}_{1}(0)}|\xi| J(|\xi|)\left(D_{\boldsymbol{e}}^{\epsilon_{n}|\xi|} \boldsymbol{u}^{\epsilon_{n}} \cdot \boldsymbol{e}\right)^{+} d \xi \varphi(\boldsymbol{x}) d \boldsymbol{x}
\end{aligned}
$$


to conclude

$$
\begin{aligned}
& \lim _{\epsilon_{n} \rightarrow 0} \int_{D \times \mathcal{H}_{1}(0)}|\xi| J(|\xi|)\left(D_{\boldsymbol{e}}^{\epsilon_{n}|\xi|} \boldsymbol{u}^{\epsilon_{n}} \cdot \boldsymbol{e}\right)^{+} d \xi \varphi(\boldsymbol{x}) d \boldsymbol{x} \\
& \quad=C \int_{\mathcal{J}_{u^{0}(t)}}\left[\boldsymbol{u}^{0}\right] \cdot \boldsymbol{n} \varphi d \mathcal{H}^{1}(\boldsymbol{x}) .
\end{aligned}
$$

On changing variables we obtain the identities:

$$
\begin{aligned}
& \lim _{\epsilon_{n} \rightarrow 0} \frac{1}{\epsilon_{n}^{2}} \int_{D} \int_{\mathcal{H}_{\epsilon_{n}}(\boldsymbol{x})} \frac{|\boldsymbol{y}-\boldsymbol{x}|}{\epsilon_{n}} J^{\epsilon_{n}}(|\boldsymbol{y}-\boldsymbol{x}|) S\left(\boldsymbol{y}, \boldsymbol{x}, \boldsymbol{u}^{\epsilon_{n}}(t)\right)^{+} d \boldsymbol{y} \varphi(\boldsymbol{x}) d \boldsymbol{x} \\
& \quad=C \int_{\mathcal{J}_{u^{0}(t)}}\left[\boldsymbol{u}^{0}\right] \cdot \boldsymbol{n} \varphi d \mathcal{H}^{1}(\boldsymbol{x}) .
\end{aligned}
$$

and

$$
\begin{aligned}
\lim _{\epsilon_{n} \rightarrow 0} & \frac{1}{\epsilon_{n}^{2}} \int_{D} \int_{\mathcal{H}_{\epsilon_{n}}(\boldsymbol{x})} \frac{|\boldsymbol{y}-\boldsymbol{x}|}{\epsilon_{n}} J^{\epsilon_{n}}(|\boldsymbol{y}-\boldsymbol{x}|) S\left(\boldsymbol{y}, \boldsymbol{x}, \boldsymbol{u}^{\epsilon_{n}}(t)\right)^{-} d \boldsymbol{y} \varphi(\boldsymbol{x}) d \boldsymbol{x} \\
& =C \int_{D} \operatorname{div}^{0}(\boldsymbol{x}) \varphi(\boldsymbol{x}) d \boldsymbol{x},
\end{aligned}
$$

and Lemma 5.1 is proved.

To prove Theorem 3.1 note first that the sequence $\left\{\boldsymbol{u}^{\epsilon}\right\}_{\epsilon>0}$ converges in $L^{2}\left(D, \mathbb{R}^{2}\right)$ to $\boldsymbol{u}^{0} \in S B D^{2}(D)$ and $\boldsymbol{u}^{0} \in \dot{L}^{2}\left(D ; \mathbb{R}^{2}\right)$. On passage to a subsequence if necessary it is seen that that $\left\{\boldsymbol{u}^{\epsilon}\right\}_{\epsilon>0}$ converges almost everywhere to $\boldsymbol{u}^{0}$. Since the subsequence $u_{1}^{\epsilon}$ is even with respect to $x_{2}=0$ it is evident from (5.1) that $u_{1}^{0}$ is also even, a.e. with respect to two dimensional Lebesgue measure and from (5.2) does not jump across the $x_{2}=0$ axis. Similarly since the subsequence $u_{2}^{\epsilon}$ is odd we find that $u_{2}^{0}$ is odd a.e. with respect to two dimensional Lebesgue measure. From (2.31) and (5.11) of Lemma 5.1 we see that choosing any test function $\varphi$ with $\operatorname{supp}\{\varphi\} \subset \subset D_{t}$ shows that the jump set $\mathcal{J}_{\boldsymbol{u}^{0}(t)}$ is confined to the $x_{2}=0$ axis and does not intersect $\left\{\ell^{0}(t)<x_{1}<a, x_{2}=0\right\}$. It now follows from (5.9) that $u_{2}^{0}=0$ a.e. on $\left\{\ell^{0}(t)<x_{1}<a, x_{2}=0\right\}$ with respect to one dimensional $\mathcal{H}^{1}$ measure. Now let $D^{ \pm}=\left\{\boldsymbol{x} \in D: \pm x_{2}>0\right\}$ then on applying Korn's inequality, there is a positive constant $C$ for which

$$
\left\|\boldsymbol{u}^{0}\right\|_{L^{2}\left(D^{ \pm}, \mathbb{R}^{2}\right)}^{2}+\int_{D^{ \pm}}\left|\nabla \boldsymbol{u}^{0}\right|^{2} d \boldsymbol{x} \leq C\left(\left\|\boldsymbol{u}^{0}\right\|_{L^{2}\left(D^{ \pm}, \mathbb{R}^{2}\right)}^{2}+\int_{D^{ \pm}}\left|\mathcal{E} \boldsymbol{u}^{0}\right|^{2} d \boldsymbol{x}\right)
$$

so $\boldsymbol{u}^{0} \in \dot{H}^{1}\left(D_{t}, \mathbb{R}^{2}\right)$ and the theorem is established.

\section{Convergence of nonlocal elastodynamics}

In this section we give the proofs of Lemmas 3.1, 3.2, 3.3, 3.4, and Theorems 3.2 and 3.3. We begin with the derivation of Theorem 3.3. This is done with the aid of the following variational identities over properly chosen test spaces. The first variational identity over the domain $D$ is given in the following lemma. 
Lemma 6.1. For a.e. $t \in(0, T)$ we have

$$
\rho\left\langle\ddot{\boldsymbol{u}}^{0}, \boldsymbol{w}\right\rangle=-\int_{D} \mathbb{C} \mathcal{E} \boldsymbol{u}^{0}: \mathcal{E} \boldsymbol{w} d x, \text { for all } \boldsymbol{w} \in H_{0}^{1}\left(D, \mathbb{R}^{2}\right),
$$

where $\langle\cdot, \cdot\rangle$ is the duality paring between $H_{0}^{1}\left(D, \mathbb{R}^{2}\right)$ and $H^{-1}\left(D, \mathbb{R}^{2}\right)$.

The next variational identity includes the traction at the domain outer boundary.

Lemma 6.2. For a.e. $t \in(0, T)$ we have

$$
\rho\left\langle\ddot{\boldsymbol{u}}^{0}, \boldsymbol{w}\right\rangle=-\int_{D} \mathbb{C} \mathcal{E} \boldsymbol{u}^{0}: \mathcal{E} \boldsymbol{w} d x+\int_{\partial D} \boldsymbol{g} \cdot \boldsymbol{w} d \sigma, \text { for all } \quad \boldsymbol{w} \in \dot{H}^{1}\left(D, \mathbb{R}^{2}\right),
$$

where $\langle\cdot, \cdot\rangle$ is the duality paring between $\dot{H}^{1}\left(D, \mathbb{R}^{2}\right)$ and its Hilbert space dual $\dot{H}^{1}\left(D, \mathbb{R}^{2}\right)^{\prime}$.

The next variational identity applies to the domains $L_{\beta}^{ \pm}(t)$ adjacent to the boundary and set apart from the crack tip.

Lemma 6.3. Given that the field $\ddot{\boldsymbol{u}}_{\tau}^{0}(t)$ is a bounded linear functional on the spaces $W^{ \pm}\left(D_{\beta}(\tau)\right)$ for a.e. $t \in(\tau, T)$ we also have the identity

$$
\begin{gathered}
\rho\left\langle\ddot{\boldsymbol{u}}_{\tau}^{0}, \boldsymbol{w}\right\rangle=-\int_{L_{\beta}^{ \pm}(\tau)} \mathbb{C} \mathcal{E} \boldsymbol{u}^{0}: \mathcal{E} \boldsymbol{w} d \boldsymbol{x}+\int_{\partial D_{\beta}^{ \pm}(\tau)} \boldsymbol{g} \cdot \boldsymbol{w} d \sigma, \\
\text { for all } \boldsymbol{w} \in W^{ \pm}\left(D_{\beta}(\tau)\right) .
\end{gathered}
$$

We now prove Theorem 3.3 using Lemmas 3.3 and 3.4 and the variational identities given above by Lemmas 6.1, 6.2 and 6.3. It is evident from Lemma 6.1 that

$$
\rho \ddot{\boldsymbol{u}}^{0}=\operatorname{div}\left(\mathbb{C} \mathcal{E} \boldsymbol{u}^{0}\right)
$$

as elements of $H^{-1}\left(D, \mathbb{R}^{2}\right)$ and (3.14) of Theorem 3.3 is established. The traction on $\partial D$ given by (3.15) now follows immediately from Lemmas 3.3 and 6.2. Similarly the zero traction force acting on the component of $\partial D_{\beta}(\tau)^{ \pm}$lying on the crack faces given by (3.16) now follows immediately from Lemmas 3.4 and 6.3. This concludes the proof of Theorem 3.3.

Lemmas 3.3 and 3.4 will be shown to follow from a generalized trace formula on the boundary of a Lipschitz domain $\Omega$. We call the domain $\Omega$ a polygon when it is a Lipschitz domain with smooth curvilinear arcs for edges $E_{i}, i=1, \ldots M$, connected by vertices. We introduce the Sobolev space defined on $\Omega$ given by

$$
H^{1,0}\left(\Omega, \mathbb{R}^{2}\right)=\left\{\boldsymbol{w} \in H^{1}\left(\Omega, \mathbb{R}^{2}\right) \text { and } \gamma \boldsymbol{w}=0 \text { on a subset of edges }\right\},
$$
here $H^{1,0}\left(\Omega, \mathbb{R}^{2}\right) \subset \dot{H}^{1}\left(\Omega, \mathbb{R}^{2}\right)$.

Lemma 6.4. Given a domain $\Omega$ with Lipschitz boundary and let $\boldsymbol{u}^{0}$ be an element of $\dot{H}^{1}\left(\Omega ; \mathbb{R}^{2}\right)$, let $\boldsymbol{f}$ be an element of $\dot{H}^{1}\left(\Omega, \mathbb{R}^{2}\right)^{\prime}$, and

$$
\operatorname{div}\left(\mathbb{C} \mathcal{E} \boldsymbol{u}^{0}\right)=\boldsymbol{f}
$$


as elements of $H^{-1}\left(\Omega, \mathbb{R}^{2}\right)$. Suppose first that test functions $\boldsymbol{w}$ belong to $\dot{H}^{1}$ $\left(\Omega, \mathbb{R}^{2}\right)$ and define $\mathbb{C} \mathcal{E} \boldsymbol{u}^{0} \boldsymbol{n}$ on $\partial \Omega$ by

$$
\left\langle\mathbb{C} \mathcal{E} \boldsymbol{u}^{0} \boldsymbol{n}, \gamma \boldsymbol{w}\right\rangle=\int_{\Omega} \mathbb{C} \mathcal{E} \boldsymbol{u}^{0}: \mathcal{E} \boldsymbol{w} d \boldsymbol{x}+\langle\boldsymbol{f}, \boldsymbol{w}\rangle
$$

for all $\boldsymbol{w}$ in $\dot{H}^{1}\left(\Omega, \mathbb{R}^{2}\right)$. Then the functional $\left\langle\mathbb{C} \mathcal{E} \boldsymbol{u}^{0} \boldsymbol{n}, \gamma \boldsymbol{w}\right\rangle$ is uniquely defined for all test functions $\boldsymbol{w}$ in $\dot{H}^{1}\left(\Omega, \mathbb{R}^{2}\right)$, hence $\mathbb{C} \mathcal{E} \boldsymbol{u}^{0} \boldsymbol{n}$ belongs to $H^{-1 / 2}(\partial \Omega)$.

Next suppose $\Omega$ is a polygon. Let $\boldsymbol{w}$ belong to $H^{1,0}\left(\Omega, \mathbb{R}^{2}\right)$ and let $\boldsymbol{f}$ be an element of $H^{1,0}\left(\Omega, \mathbb{R}^{2}\right)^{\prime}$ and let div $\left(\mathbb{C} \mathcal{E} \boldsymbol{u}^{0}\right)$ and $\boldsymbol{f}$ satisfy $(6.6)$ as elements of $H^{-1}\left(\Omega, \mathbb{R}^{2}\right)$. Define $\mathbb{C} \mathcal{E} \boldsymbol{u}^{0} \boldsymbol{n}$ on $\partial \Omega$ by

$$
\left\langle\mathbb{C} \mathcal{E} \boldsymbol{u}^{0} \boldsymbol{n}, \gamma \boldsymbol{w}\right\rangle=\int_{\Omega} \mathbb{C} \mathcal{E} \boldsymbol{u}^{0}: \mathcal{E} \boldsymbol{w} d \boldsymbol{x}+\langle\boldsymbol{f}, \boldsymbol{w}\rangle
$$

for all $\boldsymbol{w}$ in $H^{1,0}\left(\Omega, \mathbb{R}^{2}\right)$. The functional $\left\langle\mathbb{C} \mathcal{E} \boldsymbol{u}^{0} \boldsymbol{n}, \gamma \boldsymbol{w}\right\rangle$ is uniquely defined for all test functions $\boldsymbol{w}$ in $H^{1,0}\left(\Omega, \mathbb{R}^{2}\right)$, hence $\mathbb{C} \mathcal{E} \boldsymbol{u}^{0} \boldsymbol{n}$ belongs to the dual space $H_{00}^{-1 / 2}(\partial \Omega)$.

We now prove Lemmas 3.3 and 3.4. From Lemma 6.1

$$
\rho \ddot{\boldsymbol{u}}^{0}=\operatorname{div}\left(\mathbb{C} \mathcal{E} \boldsymbol{u}^{0}\right),
$$

as elements of $H^{-1}\left(\Omega, \mathbb{R}^{2}\right)$. Then we set $\boldsymbol{f}=\rho \ddot{\boldsymbol{u}}^{0}$ and Lemma 3.3 follows immediately from the first part of Lemma 6.4. Now we see that the domains $L_{\beta}^{ \pm}(t)$ of Lemma 3.4 are polygons. With the hypothesis of lemma 3.4 we apply Lemma 6.3 and first consider test functions $\boldsymbol{w}$ in $W^{ \pm}\left(D_{\beta}(\tau)\right)$ that vanish on the boundary of $L_{\beta}^{ \pm}(t)$. Substitution into (6.3) gives

$$
\rho \ddot{\boldsymbol{u}}_{\tau}^{0}=\operatorname{div}\left(\mathbb{C} \mathcal{E} \boldsymbol{u}^{0}\right),
$$

as elements of $H^{-1}\left(L_{\beta}^{ \pm}(t), \mathbb{R}^{2}\right)$. Note that $\boldsymbol{w} \in W^{ \pm}\left(D_{\beta}(t)\right)$ implies that the restriction of $\boldsymbol{w}$ to $L_{\beta}^{ \pm}(t)$ belongs to

$$
H^{1,0}\left(L_{\beta}^{ \pm}(t), \mathbb{R}^{2}\right)=\left\{\boldsymbol{w} \in H^{1}\left(L_{\beta}^{ \pm}(t), \mathbb{R}^{2}\right) \text { and } \gamma \boldsymbol{w}=0 \text { on } \partial L^{ \pm}\right\},
$$

so we set we set $\boldsymbol{f}=\rho \ddot{\boldsymbol{u}}^{0}$ and Lemma 3.4 follows immediately from the second part of Lemma 6.4.

We now prove the lemmas introduced in this section. We begin with the proof of Lemma 6.4 following [28]. To fix ideas we prove the second part of Lemma 6.4 noting the first part follows identical lines. First note if $\boldsymbol{u}^{0}$ belongs to $H^{1}\left(\Omega ; \mathbb{R}^{2}\right)$ then $\int_{\Omega} \mathbb{C} \mathcal{E} \boldsymbol{u}^{0}: \mathcal{E} \boldsymbol{w} d \boldsymbol{x}$ as a map from $\boldsymbol{w} \in H^{1,0}\left(\Omega ; \mathbb{R}^{2}\right)$ to $\mathbb{R}$ belongs to $H^{1,0}\left(\Omega ; \mathbb{R}^{2}\right)^{\prime}$. Second note that the trace operator mapping $H^{1,0}\left(\Omega ; \mathbb{R}^{2}\right)$ to $H_{00}^{-1 / 2}(\Omega)$ has a continuous right inverse denoted by $\tau$. We define $\tilde{\boldsymbol{g}}$ by

$$
\langle\tilde{\boldsymbol{g}}, \boldsymbol{v}\rangle=\int_{\Omega} \mathbb{C} \mathcal{E} \boldsymbol{u}^{0}: \mathcal{E} \tau \boldsymbol{v} d \boldsymbol{x}+\langle\boldsymbol{f}, \tau \boldsymbol{v}\rangle
$$

for all $\boldsymbol{v}$ in $H_{00}^{-1 / 2}(\partial \Omega)$ to show

$$
\langle\tilde{\boldsymbol{g}}, \gamma \boldsymbol{w}\rangle=\int_{\Omega} \mathbb{C} \mathcal{E} \boldsymbol{u}^{0}: \mathcal{E} \boldsymbol{w} d \boldsymbol{x}+\langle\boldsymbol{f}, \boldsymbol{w}\rangle
$$


for all $\boldsymbol{w}$ in $H^{1,0}\left(\Omega ; \mathbb{R}^{2}\right)$. To see this pick $\boldsymbol{w}$ in $H^{1,0}\left(\Omega ; \mathbb{R}^{2}\right)$ and set $\boldsymbol{w}_{0}=$ $\boldsymbol{w}-\tau \gamma \boldsymbol{w}$ so $\boldsymbol{w}_{0}$ is in $H_{0}^{1}\left(\Omega ; \mathbb{R}^{2}\right)$ and from (6.6) we have

$$
-\int_{\Omega} \mathbb{C} \mathcal{E} \boldsymbol{u}^{0}: \mathcal{E} \boldsymbol{w}_{0} d \boldsymbol{x}=\left\langle\boldsymbol{w}_{0}, \boldsymbol{f}\right\rangle,
$$

SO

$$
-\int_{\Omega} \mathbb{C} \mathcal{E} \boldsymbol{u}^{0}: \mathcal{E} \boldsymbol{w} d \boldsymbol{x}+\int_{\Omega} \mathbb{C} \mathcal{E} \boldsymbol{u}^{0}: \mathcal{E} \tau \gamma \boldsymbol{w} d \boldsymbol{x}=\langle\boldsymbol{w}, \boldsymbol{f}\rangle-\langle\tau \gamma \boldsymbol{w}, \boldsymbol{f}\rangle .
$$

Equation (6.13) follows directly from (6.15), (6.12), and manipulation. Now we show that the definition of $\tilde{\boldsymbol{g}}$ given by (6.12) is unique and independent of the choice of right inverse (lift) $\tau$. Suppose we have $\boldsymbol{g}^{*}$ defined by the lift $\tau^{*}$ given by

$$
\left\langle\boldsymbol{g}^{*}, \boldsymbol{v}\right\rangle=\int_{\Omega} \mathbb{C} \mathcal{E} \boldsymbol{u}^{0}: \mathcal{E} \tau^{*} \boldsymbol{v} d \boldsymbol{x}+\left\langle\boldsymbol{f}, \tau^{*} \boldsymbol{v}\right\rangle
$$

for all $\boldsymbol{v}$ in $H_{00}^{-1 / 2}(\partial \Omega)$. From (6.13) and linearity we get

$$
\left\langle\tilde{\boldsymbol{g}}-\boldsymbol{g}^{*}, \gamma \boldsymbol{w}\right\rangle=0
$$

for all $\boldsymbol{w}$ in $H^{1,0}\left(\Omega, \mathbb{R}^{2}\right)$ and uniqueness follows. We define $\mathbb{C} \mathcal{E} \boldsymbol{u}^{0} \boldsymbol{n}=\tilde{\boldsymbol{g}}$ and the second part of Lemma 6.4 is proved.

Next we give the proof of Lemma 3.1. First we show that the sequence $\left\{\boldsymbol{b}^{\epsilon_{n}}(t)\right\}$ is uniformly bounded in $H^{1}\left(D ; \mathbb{R}^{2}\right)^{\prime}$ for $t \in[0, T]$. Let $\chi^{\epsilon_{n}}=\chi_{+}^{\epsilon_{n}}+\chi_{-}^{\epsilon_{n}}$ where $\chi_{ \pm}^{\epsilon_{n}}$ are the indicator functions of the body force layers defined in (2.10) so recalling (2.11) then for any $\boldsymbol{w} \in H^{1}\left(D ; \mathbb{R}^{2}\right)$, we have

$$
\begin{aligned}
& \int_{D} \boldsymbol{b}^{\epsilon_{n}}(\boldsymbol{x}, t) \cdot \boldsymbol{w}(\boldsymbol{x}) d \boldsymbol{x}=\int_{D} \frac{1}{\epsilon_{n}} \chi^{\epsilon_{n}}(\boldsymbol{x}) \boldsymbol{g}\left(x_{1}, t\right) \cdot \boldsymbol{w}(\boldsymbol{x}) d \boldsymbol{x} \\
& =\int_{D} \frac{1}{\sqrt{\epsilon_{n}}} \chi^{\epsilon_{n}}(\boldsymbol{x}) \boldsymbol{g}\left(x_{1}, t\right) \cdot \frac{1}{\sqrt{\epsilon_{n}}} \chi^{\epsilon_{n}}(\boldsymbol{x}) \boldsymbol{w}(\boldsymbol{x}) d \boldsymbol{x} \\
& \leq\left(\int_{D} \frac{1}{\epsilon_{n}} \chi^{\epsilon_{n}}|\boldsymbol{g}(t)|^{2} d \boldsymbol{x}\right)^{1 / 2}\left(\int_{D} \frac{1}{\epsilon_{n}} \chi^{\epsilon_{n}}(\boldsymbol{x})|\boldsymbol{w}|^{2} d \boldsymbol{x}\right)^{1 / 2} \\
& \leq 2\|g(t)\|_{L^{2}(\theta, a-\theta)} I_{\epsilon_{n}} .
\end{aligned}
$$

Here $I_{\epsilon_{n}}$ is given by

$$
\begin{aligned}
I_{\epsilon_{n}}= & \left(\int_{D} \frac{1}{\epsilon_{n}} \chi^{\epsilon_{n}}(\boldsymbol{x})|\boldsymbol{w}|^{2} d \boldsymbol{x}\right)^{1 / 2} \\
= & \left(\int_{0}^{1} \int_{\theta}^{a-\theta}\left|\boldsymbol{w}\left(x_{1}, \frac{b}{2}+\epsilon_{n}\left(y_{2}-1\right)\right)\right|^{2} d x_{1} d y_{2}\right. \\
& +\int_{0}^{1} \int_{\theta}^{a-\theta} \mid \boldsymbol{w}\left(x_{1},-\frac{b}{2}+\left.\epsilon_{n}\left(1-y_{2}\right)\right|^{2} d x_{1} d y_{2}\right)^{1 / 2}
\end{aligned}
$$


where the change of variables $x_{2}= \pm \frac{b}{2} \mp \epsilon_{n} \pm \epsilon_{n} y_{2}$ has been made. From the change of variable it is evident that the factor $I_{\epsilon_{n}}$ is bounded above by

$$
I_{\epsilon_{n}} \leq\left(\int_{0}^{1} \int_{\partial D_{\delta(y)}}|\boldsymbol{w}|^{2} d s d y\right)^{1 / 2}
$$

where $D_{\delta(y)}=\{\boldsymbol{x} \in D: \operatorname{dist}(\boldsymbol{x}, \partial D)>\delta(y)\}$ and $\delta(y)=\epsilon_{n}(1-y)$, for $0<y<1$. Since the trace operator is a bounded linear transformation between $H^{1}\left(D_{\delta(y)}, \mathbb{R}^{2}\right)$ and $L^{2}\left(\partial D_{\delta(y)}\right)^{2}$ we have

$$
\int_{\partial D_{\delta(y)}}|\boldsymbol{w}|^{2} d s \leq C_{\delta(y)}\|\boldsymbol{w}\|_{H^{1}\left(D_{\delta(y)}, \mathbb{R}^{2}\right)}^{2} \leq C_{\delta(y)}\|\boldsymbol{w}\|_{H^{1}\left(D, \mathbb{R}^{2}\right)}^{2} .
$$

Additionally $C_{\delta(y)}$ depends only on the Lipschitz constant of the boundary [16] so for the case at hand we see that

$$
\sup _{y \in[0,1]}\left\{C_{\delta(y)}\right\}<\infty,
$$

and from (6.18), (6.20), and (6.22) we conclude that there is a constant $C$ independent of $t$ and $\epsilon_{n}$ such that

$$
\left|\int_{D} \boldsymbol{b}^{\epsilon_{n}}(\boldsymbol{x}, t) \cdot \boldsymbol{w}(\boldsymbol{x}) d \boldsymbol{x}\right| \leq C\|\boldsymbol{w}\|_{H^{1}\left(D, \mathbb{R}^{2}\right)}^{2},
$$

and

$$
\sup _{\epsilon_{n}>0} \int_{0}^{T}\left\|\boldsymbol{b}^{\epsilon_{n}}(t)\right\|_{H^{1}\left(D ; \mathbb{R}^{2}\right)^{\prime}}^{2} d t<\infty .
$$

Thus we can pass to a subsequence also denoted by $\left\{\boldsymbol{b}^{\epsilon_{n}}\right\}_{n=1}^{\infty}$ that converges weakly to $\boldsymbol{b}^{0}$ in $L^{2}\left(0, T ; H^{1}\left(D ; \mathbb{R}^{2}\right)^{\prime}\right)$. Next we identify the weak limit $\boldsymbol{b}^{0}(t)$ for a dense set of trial fields. Let $\boldsymbol{w} \in C^{1}\left(\bar{D}, \mathbb{R}^{2}\right)$ then a change of variables $x_{2}= \pm \frac{b}{2} \mp \epsilon_{n} \pm \epsilon_{n} y_{2}$ gives

$$
\begin{aligned}
\int_{D} \boldsymbol{b}^{\epsilon_{n}}(\boldsymbol{x}, t) \cdot \boldsymbol{w}(\boldsymbol{x}) d \boldsymbol{x}= & \int_{D} \frac{1}{\epsilon_{n}} \chi^{\epsilon_{n}}(\boldsymbol{x}) \boldsymbol{g}\left(x_{1}, t\right) \cdot \boldsymbol{w}(\boldsymbol{x}) d \boldsymbol{x} \\
= & \int_{0}^{1} \int_{\theta}^{a-\theta} g\left(x_{1}, t\right) \boldsymbol{e}^{2} \cdot \boldsymbol{w}\left(x_{1}, \frac{b}{2}+\epsilon_{n}\left(y_{2}-1\right) d x_{1} d y_{2}\right. \\
& -\int_{0}^{1} \int_{\theta}^{a-\theta} g\left(x_{1}, t\right) \boldsymbol{e}^{2} \cdot \boldsymbol{w}\left(x_{1},-\frac{b}{2}+\epsilon_{n}\left(1-y_{2}\right) d x_{1} d y_{2} .\right.
\end{aligned}
$$

One passes to the $\epsilon_{n} \rightarrow 0$ limit in (6.25) applying the uniform continuity of $\boldsymbol{w}$ to obtain

$$
\lim _{\epsilon_{n} \rightarrow 0} \int_{D} \boldsymbol{b}^{\epsilon_{n}}(\boldsymbol{x}, t) \cdot \boldsymbol{w}(\boldsymbol{x}) d \boldsymbol{x}=\int_{\partial D} \boldsymbol{g} \cdot \boldsymbol{w} d \sigma .
$$

Lemma 3.1 now follows noting that $C^{1}\left(\bar{D}, \mathbb{R}^{2}\right)$ is dense in $H^{1}\left(D, \mathbb{R}^{2}\right)$.

We now establish Lemma 3.2. The strong convergence

$$
\boldsymbol{u}^{\epsilon_{n}} \rightarrow \boldsymbol{u}^{0} \text { strong in } C\left([0, T] ; \dot{L}^{2}\left(D ; \mathbb{R}^{2}\right)\right)
$$


follows immediately from the same arguments used to establish theorem 5.1 of [24]. The weak convergence

$$
\dot{\boldsymbol{u}}^{\epsilon_{n}} \rightarrow \dot{\boldsymbol{u}}^{0} \text { weakly in } L^{2}\left(0, T ; \dot{L}^{2}\left(D ; \mathbb{R}^{2}\right)\right)
$$

follows noting that theorem 2.2 of [24] shows that

$$
\sup _{\epsilon_{n}>0} \int_{0}^{T}\left\|\dot{\boldsymbol{u}}^{\epsilon_{n}}(t)\right\|_{L^{2}\left(D ; \mathbb{R}^{2}\right)}^{2} d t<\infty .
$$

Thus we can pass to a subsequence also denoted by $\left\{\dot{\boldsymbol{u}}^{\epsilon_{n}}\right\}_{n=1}^{\infty}$ that converges weakly to $\dot{\boldsymbol{u}}^{0}$ in $L^{2}\left(0, T ; \dot{L}^{2}\left(D ; \mathbb{R}^{2}\right)\right)$.

To prove

$$
\ddot{\boldsymbol{u}}^{\epsilon_{n}} \rightarrow \ddot{\boldsymbol{u}}^{0} \text { weakly in } L^{2}\left(0, T ; \dot{H}^{1}\left(D ; \mathbb{R}^{2}\right)^{\prime}\right)
$$

we must show that

$$
\sup _{\epsilon_{n}>0} \int_{0}^{T}\left\|\ddot{\boldsymbol{u}}^{\epsilon_{n}}(t)\right\|_{H^{1}\left(D ; \mathbb{R}^{2}\right)^{\prime}}^{2} d t<\infty,
$$

and existence of a weakly converging sequence follows. We multiply (2.16) with a test function $\boldsymbol{w}$ from $\dot{H}^{1}\left(D ; \mathbb{R}^{2}\right)$ and integrate over $D$.

A straightforward integration by parts gives

$$
\begin{aligned}
\int_{D} \ddot{\boldsymbol{u}}^{\epsilon_{n}}(\boldsymbol{x}, t) \cdot \boldsymbol{w}(\boldsymbol{x}) d \boldsymbol{x} \\
=-\frac{1}{\rho} \int_{D} \int_{\mathcal{H}_{\epsilon_{n}}(\boldsymbol{x}) \cap D}|\boldsymbol{y}-\boldsymbol{x}| \partial_{S} \mathcal{W}^{\epsilon_{n}}\left(\boldsymbol{y}-\boldsymbol{x}, S\left(\boldsymbol{y}, \boldsymbol{x}, \boldsymbol{u}^{\epsilon_{n}}(t)\right)\right) S(\boldsymbol{y}, \boldsymbol{x}, \boldsymbol{w}) d \boldsymbol{y} d \boldsymbol{x} \\
\quad+\frac{1}{\rho} \int_{D} \boldsymbol{b}^{\epsilon_{n}}(\boldsymbol{x}, t) \cdot \boldsymbol{w}(\boldsymbol{x}) d \boldsymbol{x}
\end{aligned}
$$

and we now estimate the right hand side of (6.32). The first term on the righthand side is denoted by $I^{\epsilon_{n}}$ and we change variables $\boldsymbol{y}=\boldsymbol{x}+\epsilon \xi,|\xi|<1$, with $d \boldsymbol{y}=\epsilon_{n}^{2} d \xi$ and write out $\partial_{S} \mathcal{W}^{\epsilon}\left(\boldsymbol{y}-\boldsymbol{x}, S\left(\boldsymbol{y}, \boldsymbol{x}, \boldsymbol{u}^{\epsilon}(t)\right)\right)$ to get

$$
\begin{aligned}
I^{\epsilon_{n}}= & -\frac{1}{\rho \omega_{2}} \int_{D \times \mathcal{H}_{1}(0)} \omega\left(\boldsymbol{x}, \epsilon_{n} \xi\right)|\xi| J(|\xi|) h^{\prime}\left(\epsilon_{n}|\xi|\left|D_{\boldsymbol{e}}^{\epsilon_{n}|\xi|} \boldsymbol{u}^{\epsilon_{n}} \cdot \boldsymbol{e}\right|^{2}\right) \\
& \times 2\left(D_{\boldsymbol{e}}^{\epsilon_{n}|\xi|} \boldsymbol{u}^{\epsilon_{n}} \cdot \boldsymbol{e}\right)\left(D_{\boldsymbol{e}}^{\epsilon_{n}|\xi|} \boldsymbol{w} \cdot \boldsymbol{e}\right) d \xi d \boldsymbol{x},
\end{aligned}
$$

where $\omega\left(\boldsymbol{x}, \epsilon_{n} \xi\right)$ is unity if $\boldsymbol{x}+\epsilon_{n} \xi$ is in $D$ and zero otherwise. We define the sets

$$
\begin{aligned}
& A_{\epsilon_{n}}^{-}=\left\{(\boldsymbol{x}, \xi) \text { in } D \times \mathcal{H}_{1}(0) ;\left|D_{e}^{\epsilon_{n}|\xi|} \boldsymbol{u}^{\epsilon_{n}} \cdot \boldsymbol{e}\right|<\frac{r^{c}}{\sqrt{\epsilon_{n}|\xi|}}\right\} \\
& A_{\epsilon_{n}}^{+}=\left\{(\boldsymbol{x}, \xi) \text { in } D \times \mathcal{H}_{1}(0) ;\left|D_{e}^{\epsilon_{n}|\xi|} \boldsymbol{u}^{\epsilon_{n}} \cdot \boldsymbol{e}\right| \geq \frac{r^{c}}{\sqrt{\epsilon_{n}|\xi|}}\right\},
\end{aligned}
$$

with $D \times \mathcal{H}_{1}(0)=A_{\epsilon_{n}}^{-} \cup A_{\epsilon_{n}}^{+}$. Note that $(\boldsymbol{x}, \xi) \in A_{\epsilon_{n}}^{+}$is equivalent to $(\boldsymbol{x}, \boldsymbol{y}) \in$ $S Z^{\epsilon_{n}}$ and $(\boldsymbol{x}, \xi) \in A_{\epsilon_{n}}^{-}$is equivalent to $(\boldsymbol{x}, \boldsymbol{y}) \notin S Z^{\epsilon_{n}}$. We write

$$
I^{\epsilon_{n}}=I_{1}^{\epsilon_{n}}+I_{2}^{\epsilon_{n}},
$$


where

$$
\begin{aligned}
I_{1}^{\epsilon_{n}}= & -\frac{1}{\rho \omega_{2}} \int_{D \times \mathcal{H}_{1}(0) \cap A_{\epsilon_{n}}^{-}} \omega\left(\boldsymbol{x}, \epsilon_{n} \xi\right)|\xi| J(|\xi|) h^{\prime}\left(\epsilon_{n}|\xi|\left|D_{\boldsymbol{e}}^{\epsilon_{n}|\xi|} \boldsymbol{u}^{\epsilon_{n}} \cdot \boldsymbol{e}\right|^{2}\right) \\
& \times 2\left(D_{e}^{\epsilon_{n}|\xi|} \boldsymbol{u}^{\epsilon_{n}} \cdot \boldsymbol{e}\right)\left(D_{\boldsymbol{e}}^{\epsilon_{n}|\xi|} \boldsymbol{w} \cdot \boldsymbol{e}\right) d \xi d \boldsymbol{x} \\
I_{2}^{\epsilon_{n}}= & -\frac{1}{\rho \omega_{2}} \int_{D \times \mathcal{H}_{1}(0) \cap A_{\epsilon_{n}}^{+}} \omega\left(\boldsymbol{x}, \epsilon_{n} \xi\right)|\xi| J(|\xi|) h^{\prime}\left(\epsilon_{n}|\xi|\left|D_{\boldsymbol{e}}^{\epsilon_{n}|\xi|} \boldsymbol{u}^{\epsilon_{n}} \cdot \boldsymbol{e}\right|^{2}\right) \\
& \times 2\left(D_{\boldsymbol{e}}^{\epsilon_{n}|\xi|} \boldsymbol{u}^{\epsilon_{n}} \cdot \boldsymbol{e}\right)\left(D_{\boldsymbol{e}}^{\epsilon_{n}|\xi|} \boldsymbol{w} \cdot \boldsymbol{e}\right) d \xi d \boldsymbol{x}
\end{aligned}
$$

In what follows we will denote positive constants independent of $\boldsymbol{u}^{\epsilon_{n}}$ and $\boldsymbol{w} \in$ $\dot{H}^{1}\left(D ; \mathbb{R}^{2}\right)$ by $C$. First note that $h$ is concave so $h^{\prime}(r)$ is monotone decreasing for $r \geq 0$ and from Cauchy's inequality, and (5.18) one has

$$
\begin{aligned}
\left|I_{1}^{\epsilon_{n}}\right| & \left.\leq\left.\frac{2 h^{\prime}(0) C}{\rho \omega_{2}}\left(\int_{D \times \mathcal{H}_{1}(0) \cap A_{\epsilon_{n}}^{-}} \omega\left(\boldsymbol{x}, \epsilon_{n} \xi\right) \mid D_{\boldsymbol{e}}^{\epsilon_{n}|\xi|} \boldsymbol{w} \cdot \boldsymbol{e}\right)\right|^{2} d \xi d \boldsymbol{x}\right)^{1 / 2} \\
& \left.\leq\left.\frac{2 h^{\prime}(0) C}{\rho \omega_{2}}\left(\int_{\mathcal{H}_{1}(0)} \int_{D} \omega\left(\boldsymbol{x}, \epsilon_{n} \xi\right) \mid D_{\boldsymbol{e}}^{\epsilon_{n}|\xi|} \boldsymbol{w} \cdot \boldsymbol{e}\right)\right|^{2} d \boldsymbol{x} d \xi\right)^{1 / 2}
\end{aligned}
$$

Since $\boldsymbol{x}$ and $\boldsymbol{x}+\epsilon_{n} \xi$ belong to $D$ we write $\xi=|\xi| \boldsymbol{e}$ where $\boldsymbol{e}=\xi /|\xi|$ and calculation gives

$$
D_{e}^{\epsilon_{n}|\xi|} \boldsymbol{w} \cdot \boldsymbol{e}=\int_{0}^{1} \mathcal{E} \boldsymbol{w}\left(\boldsymbol{x}+s \epsilon_{n}|\xi| \boldsymbol{e}\right) \boldsymbol{e} \cdot \boldsymbol{e} d s
$$

with $\boldsymbol{x}+s \epsilon_{n}|\xi| \boldsymbol{e} \in D$ for $0<s<1$. Next introduce $\chi_{D}\left(\boldsymbol{x}+s \epsilon_{n}|\xi| \boldsymbol{e}\right)$ taking the value 1, if $\boldsymbol{x}+s \epsilon_{n}|\xi| \boldsymbol{e} \in D$ and 0 otherwise. Substitution of (6.38) into (6.37) and application of the Jensen inequality and Fubini's theorem gives

$$
\left|I_{1}^{\epsilon_{n}}\right| \leq \frac{2 h^{\prime}(0) C}{\rho \omega_{2}}\left(\int_{0}^{1} \int_{\mathcal{H}_{1}(0)} \int_{D} \chi_{D}\left(\boldsymbol{x}+s \epsilon_{n}|\xi| \boldsymbol{e}\right)\left|\mathcal{E} \boldsymbol{w}\left(\boldsymbol{x}+s \epsilon_{n}|\xi| \boldsymbol{e}\right) \boldsymbol{e} \cdot \boldsymbol{e}\right|^{2} d \boldsymbol{x} d \xi d s\right)^{1 / 2}
$$

and we conclude

$$
\left|I_{1}^{\epsilon_{n}}\right| \leq C\|\boldsymbol{w}\|_{H^{1}\left(D ; \mathbb{R}^{2}\right)}
$$

Elementary calculation gives the estimate (see equation (6.53) of [24])

$$
\sup _{0 \leq x<\infty}\left|h^{\prime}\left(\epsilon_{n}|\xi| x^{2}\right) 2 x\right| \leq \frac{2 h^{\prime}\left(\left(r^{c}\right)^{2}\right) r^{c}}{\sqrt{\epsilon_{n}|\xi|}}
$$

and we also have (see equation (6.78) of [24])

$$
\int_{D \times \mathcal{H}_{1}(0) \cap A_{\epsilon_{n}}^{+}} \omega(\boldsymbol{x}, \xi) J(|\xi|) d \xi d \boldsymbol{x}<C \epsilon_{n}
$$


so Cauchy's inequality and the inequalities (6.38), (6.41), (6.42) give

$$
\begin{aligned}
\left|I_{2}^{\epsilon_{n}}\right| \leq & \frac{1}{\rho \omega_{2}} \int_{D \times \mathcal{H}_{1}(0) \cap A_{\epsilon_{n}}^{+}} \omega\left(\boldsymbol{x}, \epsilon_{n} \xi\right)|\xi| J(|\xi|) \frac{2 h^{\prime}\left(\left(r^{c}\right)^{2}\right) r^{c}}{\sqrt{\epsilon_{n}|\xi|}\left|D_{e}^{\epsilon_{n}|\xi|} \boldsymbol{w} \cdot \boldsymbol{e}\right| d \xi d \boldsymbol{x}} \\
\leq & \frac{1}{\rho \omega_{2}}\left(\int_{D \times \mathcal{H}_{1}(0) \cap A_{\epsilon_{n}}^{+}} \omega\left(\boldsymbol{x}, \epsilon_{n} \xi\right)|\xi| J(|\xi|) \frac{\left(2 h^{\prime}\left(\left(r^{c}\right)^{2}\right) r^{c}\right)^{2}}{\epsilon_{n}|\xi|} d \xi d \boldsymbol{x}\right)^{1 / 2} \\
& \times\left(\int_{D \times \mathcal{H}_{1}(0) \cap A_{\epsilon_{n}}^{+}} \omega\left(\boldsymbol{x}, \epsilon_{n} \xi\right)|\xi| J(|\xi|)\left|D_{\boldsymbol{e}}^{\epsilon_{n}|\xi|} \boldsymbol{w} \cdot \boldsymbol{e}\right|^{2} d \xi d \boldsymbol{x} d t\right)^{1 / 2} \\
\leq & C\|\boldsymbol{w}\|_{H^{1}\left(D ; \mathbb{R}^{2}\right)}
\end{aligned}
$$

and we conclude that the first term on the right hand side of (6.32) admits the estimate

$$
\left|I^{\epsilon_{n}}\right| \leq\left|I_{1}^{\epsilon_{n}}\right|+\left|I_{2}^{\epsilon_{n}}\right| \leq C\|\boldsymbol{w}\|_{H^{1}\left(D ; \mathbb{R}^{2}\right)},
$$

for all $\boldsymbol{w} \in H^{1}\left(D ; \mathbb{R}^{2}\right)$.

It follows immediately from Lemma 3.1 that the second term on the right hand side of (6.32) satisfies the estimate

$$
\frac{1}{\rho}\left|\int_{D} \boldsymbol{b}^{\epsilon_{n}}(\boldsymbol{x}, t) \cdot \boldsymbol{w}(\boldsymbol{x}) d \boldsymbol{x}\right| \leq C\|\boldsymbol{w}\|_{H^{1}\left(D ; \mathbb{R}^{2}\right)}, \text { for all } \boldsymbol{w} \in H^{1}\left(D ; \mathbb{R}^{2}\right)
$$

From (6.44) and (6.45) we conclude that there exists a $C>0$ so that

$$
\left|\int_{D} \ddot{\boldsymbol{u}}^{\epsilon_{n}}(\boldsymbol{x}, t) \cdot \boldsymbol{w}(\boldsymbol{x}) d \boldsymbol{x}\right| \leq C\|\boldsymbol{w}\|_{H^{1}\left(D ; \mathbb{R}^{2}\right)} \text {, for all } \boldsymbol{w} \in \dot{H}^{1}\left(D ; \mathbb{R}^{2}\right)
$$

SO

$$
\sup _{\epsilon_{n}>0} \sup _{t \in[0, T]} \frac{\int_{D} \ddot{\boldsymbol{u}}^{\epsilon_{n}}(\boldsymbol{x}, t) \cdot \boldsymbol{w}(\boldsymbol{x}) d \boldsymbol{x}}{\|\boldsymbol{w}\|_{H^{1}\left(D ; \mathbb{R}^{2}\right)}}<C, \text { for all } \boldsymbol{w} \in \dot{H}^{1}\left(D ; \mathbb{R}^{2}\right),
$$

or

$$
\sup _{t \in[0, T]}\left\|\ddot{\boldsymbol{u}}^{\epsilon_{n}}(t)\right\|_{H^{1}\left(D ; \mathbb{R}^{2}\right)^{\prime}}<C, \text { for all } \epsilon_{n}
$$

and (6.31) follows. The estimate (6.31) implies weak compactness and passing to subsequences if necessary we deduce that $\ddot{\boldsymbol{u}}^{\epsilon_{n}} \rightarrow \ddot{\boldsymbol{u}}^{0}$ weakly in $L^{2}\left(0, T ; \dot{H}^{1}\right.$ $\left.\left(D ; \mathbb{R}^{2}\right)^{\prime}\right)$.

Finally to prove

$$
\ddot{\boldsymbol{u}}^{\epsilon_{n}} \rightarrow \ddot{\boldsymbol{u}}^{0} \text { weakly in } L^{2}\left(0, T ; H^{-1}\left(D ; \mathbb{R}^{2}\right)\right)
$$

we must show that

$$
\sup _{\epsilon_{n}>0} \int_{0}^{T}\left\|\ddot{\boldsymbol{u}}^{\epsilon_{n}}(t)\right\|_{H^{-1}\left(D ; \mathbb{R}^{2}\right)}^{2} d t<\infty
$$

and existence of a weakly converging subsequence follows. The proof of (6.49) follows precisely the same steps as the proof of (6.30). But now we multiply 
(2.16) by test functions $\boldsymbol{w} \in C_{c}^{\infty}\left(D, \mathbb{R}^{2}\right)$, and integrate over $D$. An integration by parts gives

$$
\begin{aligned}
\int_{D} \ddot{\boldsymbol{u}}^{\epsilon_{n}}(\boldsymbol{x}, t) \cdot \boldsymbol{w}(\boldsymbol{x}) d \boldsymbol{x} \\
=-\frac{1}{\rho} \int_{D} \int_{\mathcal{H}_{\epsilon_{n}}(\boldsymbol{x}) \cap D}|\boldsymbol{y}-\boldsymbol{x}| \partial_{S} \mathcal{W}^{\epsilon_{n}}\left(\boldsymbol{y}-\boldsymbol{x}, S\left(\boldsymbol{y}, \boldsymbol{x}, \boldsymbol{u}^{\epsilon_{n}}(t)\right)\right) S(\boldsymbol{y}, \boldsymbol{x}, \boldsymbol{w}) d \boldsymbol{y} d \boldsymbol{x} \\
\quad+\frac{1}{\rho} \int_{D} \boldsymbol{b}^{\epsilon_{n}}(\boldsymbol{x}, t) \cdot \boldsymbol{w}(\boldsymbol{x}) d \boldsymbol{x}
\end{aligned}
$$

Now we follow identical steps and proceed as before, here using the density of $C_{c}^{\infty}\left(D, \mathbb{R}^{2}\right)$ in $H_{0}^{1}\left(D ; \mathbb{R}^{2}\right)$, to get

$$
\sup _{t \in[0, T]}\left\|\ddot{\boldsymbol{u}}^{\epsilon_{n}}(t)\right\|_{H^{-1}\left(D ; \mathbb{R}^{2}\right)}<C, \text { for all } \epsilon_{n}
$$

and (6.50) follows. From this we conclude (6.49) and Lemma 3.2 is proved.

In what follows we first prove Lemma 6.2 noting that the proof of Lemma 6.1 follows the same steps. The proof of Lemma 6.1 is briefly summarized afterwards. To establish Lemma 6.2 we take a test function $\varphi(t) \boldsymbol{w}(\boldsymbol{x})$ with $\varphi \in$ $C_{c}^{\infty}(0, T)$ and $\boldsymbol{w}$ in $C^{\infty}\left(\bar{D}, \mathbb{R}^{2}\right)$ orthogonal to rigid body motions. Substituting this test function into (2.15) and integration by parts in time gives

$$
\begin{aligned}
\int_{0}^{T} & \varphi(t) \rho \int_{D} \ddot{\boldsymbol{u}}^{\epsilon_{n}}(\boldsymbol{x}, t) \cdot \boldsymbol{w}(\boldsymbol{x}) d \boldsymbol{x} d t \\
= & -\int_{0}^{T} \varphi(t) \int_{D} \int_{\mathcal{H}_{\epsilon_{n}}(\boldsymbol{x}) \cap D}|\boldsymbol{y}-\boldsymbol{x}| \partial_{S} \mathcal{W}^{\epsilon_{n}} \\
& \left(\boldsymbol{y}-\boldsymbol{x}, S\left(\boldsymbol{y}, \boldsymbol{x}, \boldsymbol{u}^{\epsilon_{n}}(t)\right)\right) S(\boldsymbol{y}, \boldsymbol{x} \boldsymbol{w}) d \boldsymbol{y} d \boldsymbol{x} d t \\
& +\int_{0}^{T} \varphi(t) \int_{D} \boldsymbol{b}^{\epsilon_{n}}(\boldsymbol{x}, t) \cdot \boldsymbol{w}(\boldsymbol{x}) d \boldsymbol{x} d t
\end{aligned}
$$

The goal is to pass to the $\epsilon_{n}=0$ limit in this equation to recover (6.2). The limit of the left hand side of (6.53) follows from Lemma 3.2

$$
\lim _{\epsilon_{n} \rightarrow 0} \int_{0}^{T} \varphi(t) \rho \int_{D} \ddot{\boldsymbol{u}}^{\epsilon_{n}}(\boldsymbol{x}, t) \cdot \boldsymbol{w}(\boldsymbol{x}) d \boldsymbol{x} d t=\int_{0}^{T} \varphi(t) \rho\left\langle\ddot{\boldsymbol{u}}^{0}(t), \boldsymbol{w}\right\rangle d t .
$$

To recover the $\epsilon_{n}=0$ limit of the first term on the right hand side of (6.53) we appeal to the bound (6.44) to pass to the limit under the time integral using Lebesgue dominated convergence. Next apply Lemma 6.5 of [24] to get

$$
\begin{aligned}
\lim _{\epsilon_{n} \rightarrow 0} & I^{\epsilon_{n}}=-\lim _{\epsilon_{n} \rightarrow 0} \int_{D} \int_{\mathcal{H}_{\epsilon_{n}}(\boldsymbol{x}) \cap D}|\boldsymbol{y}-\boldsymbol{x}| \partial_{S} \mathcal{W}^{\epsilon_{n}} \\
& \left(\boldsymbol{y}-\boldsymbol{x}, S\left(\boldsymbol{y}, \boldsymbol{x}, \boldsymbol{u}^{\epsilon_{n}}(t)\right)\right) S(\boldsymbol{y}, \boldsymbol{x}, \boldsymbol{w}) d \boldsymbol{y} d \boldsymbol{x} \\
= & -\lim _{\epsilon_{n} \rightarrow 0} \frac{2}{\omega_{2}} \int_{D \times \mathcal{H}_{1}(0)} \omega\left(\boldsymbol{x}, \epsilon_{n} \xi\right)|\xi| J(|\xi|) h^{\prime}(0) \\
& \left(D_{\boldsymbol{e}}^{\epsilon_{n}|\xi|} \boldsymbol{u}^{\epsilon_{n}} \cdot \boldsymbol{e}\right)^{-}\left(D_{\boldsymbol{e}}^{\epsilon_{n}|\xi|} \boldsymbol{w} \cdot \boldsymbol{e}\right) d \xi d \boldsymbol{x},
\end{aligned}
$$


where $\left(D_{e}^{\epsilon|\xi|} \boldsymbol{u}^{\epsilon} \cdot \boldsymbol{e}\right)^{-}$is defined by (5.16). As indicated in Sect. 5, $D_{\boldsymbol{e}}^{\epsilon_{n}|\xi|} \boldsymbol{u}^{\epsilon_{n}}$. $\boldsymbol{e}^{-} \rightarrow g(\boldsymbol{x}, \xi)$ converges weakly in $L^{2}\left(D \times \mathcal{H}_{1}(0), \mathbb{R}^{2}\right)$ with respect to the measure $|\xi| J(|\xi|) d \xi d \boldsymbol{x}$ and $D_{\boldsymbol{e}}^{\epsilon_{n}|\xi|} \boldsymbol{w} \cdot \boldsymbol{e} \rightarrow \mathcal{E} \boldsymbol{w} \boldsymbol{e} \cdot \boldsymbol{e}$ uniformly on $D$, so

$$
\lim _{\epsilon_{n} \rightarrow 0} I^{\epsilon_{n}}=-\frac{2}{\omega_{2}} \int_{D \times \mathcal{H}_{1}(0)} \omega\left(\boldsymbol{x}, \epsilon_{n} \xi\right)|\xi| J(|\xi|) h^{\prime}(0) g(\boldsymbol{x}, \xi) \mathcal{E} \boldsymbol{w} \boldsymbol{e} \cdot \boldsymbol{e} d \xi d \boldsymbol{x} .
$$

and from $(5.20) g(\boldsymbol{x}, \xi)=\mathcal{E} \boldsymbol{u}^{0} \boldsymbol{e} \cdot \boldsymbol{e}$ and we recover

$$
\lim _{\epsilon_{n} \rightarrow 0} I^{\epsilon_{n}}=-\int_{D} \mathbb{C} \mathcal{E} \boldsymbol{u}^{0}: \mathcal{E} \boldsymbol{w} d \boldsymbol{x}
$$

SO

$$
\lim _{\epsilon_{n} \rightarrow 0} \int_{0}^{T} \varphi(t) I^{\epsilon_{n}} d t=-\int_{0}^{T} \varphi(t) \int_{D} \mathbb{C} \mathcal{E} \boldsymbol{u}^{0}: \mathcal{E} \boldsymbol{w} d \boldsymbol{x} d t
$$

We pass to the limit in the second term on the right hand side of (6.53) using Lemma 3.1 to obtain

$$
\int_{0}^{T} \varphi(t) \rho\left\langle\ddot{\boldsymbol{u}}^{0}(t), \boldsymbol{w}\right\rangle d t=-\int_{0}^{T} \varphi(t)\left(\int_{D} \mathbb{C} \mathcal{E} \boldsymbol{u}^{0}: \mathcal{E} \boldsymbol{w} d x+\int_{\partial D} \boldsymbol{g} \cdot \boldsymbol{w} d \sigma\right) d t
$$

From the density of $C^{\infty}\left(\bar{D}, \mathbb{R}^{2}\right)$ in $\boldsymbol{w} \in \dot{H}^{1}\left(D, \mathbb{R}^{2}\right)$ we see that (6.59) holds for all $\boldsymbol{w} \in \dot{H}^{1}\left(D, \mathbb{R}^{2}\right)$. Since (6.59) holds for all $\varphi \in C_{c}^{\infty}(0, T)$ we recover (6.2).

We summarize the proof of Lemma 6.1. We multiply (6.51) on both sides by a test function $\varphi(t)$ with $\varphi \in C_{c}^{\infty}(0, T)$ and integrate in time over $(0, T)$ to get

$$
\begin{aligned}
\int_{0}^{T} & \varphi(t) \rho \int_{D} \ddot{\boldsymbol{u}}^{\epsilon_{n}}(\boldsymbol{x}, t) \cdot \boldsymbol{w}(\boldsymbol{x}) d \boldsymbol{x} d t \\
= & -\int_{0}^{T} \varphi(t) \int_{D} \int_{\mathcal{H}_{\epsilon_{n}}(\boldsymbol{x}) \cap D}|\boldsymbol{y}-\boldsymbol{x}| \partial_{S} \mathcal{W}^{\epsilon_{n}} \\
& \left(\boldsymbol{y}-\boldsymbol{x}, S\left(\boldsymbol{y}, \boldsymbol{x}, \boldsymbol{u}^{\epsilon_{n}}(t)\right)\right) S(\boldsymbol{y}, \boldsymbol{x} \boldsymbol{w}) d \boldsymbol{y} d \boldsymbol{x} d t \\
& +\int_{0}^{T} \varphi(t) \frac{1}{\rho} \int_{D} \boldsymbol{b}^{\epsilon_{n}}(\boldsymbol{x}, t) \cdot \boldsymbol{w}(\boldsymbol{x}) d \boldsymbol{x} d t
\end{aligned}
$$

We now pass to the $\epsilon_{n}=0$ limit in this equation using (6.49), (6.57) and note that the last term vanishes for any choice of $\boldsymbol{w} \in C_{c}^{\infty}\left(D, \mathbb{R}^{2}\right)$ as $\epsilon_{n} \rightarrow 0$. Last we use the density of $C_{c}^{\infty}\left(D, \mathbb{R}^{2}\right)$ in $H^{1}\left(D, \mathbb{R}^{2}\right)$ to recover (6.1) and Lemma 6.1 follows.

We now establish Theorem 3.2 to show that $\ddot{\boldsymbol{u}}_{\tau}^{0}(\boldsymbol{x}, t)$ is a bounded linear functional on the spaces $W^{ \pm}\left(D_{\beta}(\tau)\right)$ for a.e. $t \in(\tau, T)$. We illustrate the proof for $\boldsymbol{w} \in W^{+}\left(D_{\beta}(\tau)\right)$ noting that identical steps hold for $\boldsymbol{w} \in W^{-}\left(D_{\beta}(\tau)\right)$. Pick $\tau \in(0, T)$, suppose $\tau<t$, multiply (2.16) by a trial $\boldsymbol{w} \in W^{+}\left(D_{\beta}(\tau)\right)$ and 
integrating by parts over $D$ gives

$$
\begin{aligned}
\rho \int_{D} & \ddot{\boldsymbol{u}}^{\epsilon_{n}}(\boldsymbol{x}, t) \cdot \boldsymbol{w}(\boldsymbol{x}) d \boldsymbol{x} \\
= & -\int_{D} \int_{\mathcal{H}_{\epsilon_{n}}(\boldsymbol{x}) \cap D}|\boldsymbol{y}-\boldsymbol{x}| \partial_{S} \mathcal{W}^{\epsilon_{n}}\left(\boldsymbol{y}-\boldsymbol{x}, S\left(\boldsymbol{y}, \boldsymbol{x}, \boldsymbol{u}^{\epsilon_{n}}(t)\right)\right) S(\boldsymbol{y}, \boldsymbol{x}, \boldsymbol{w}) d \boldsymbol{y} d \boldsymbol{x} \\
& +\int_{D} \boldsymbol{b}^{\epsilon_{n}}(\boldsymbol{x}, t) \cdot \boldsymbol{w}(\boldsymbol{x}) d \boldsymbol{x}
\end{aligned}
$$

Now we show that $\ddot{\boldsymbol{u}}^{\epsilon_{n}}(t)$ is bounded in $W^{+}\left(D_{\beta}(\tau)\right)^{\prime}$ uniformly for all $t \in(\tau, T)$ and $0<\epsilon_{n}<\beta / 2$. As before the first term on the righthand side is denoted by $I^{\epsilon_{n}}$ and we change variables $\boldsymbol{y}=\boldsymbol{x}+\epsilon \xi,|\xi|<1$, with $d \boldsymbol{y}=\epsilon_{n}^{2} d \xi$ and write out $\partial_{S} \mathcal{W}^{\epsilon}\left(\boldsymbol{y}-\boldsymbol{x}, S\left(\boldsymbol{y}, \boldsymbol{x}, \boldsymbol{u}^{\epsilon}(t)\right)\right)$ to get

$$
\begin{aligned}
I^{\epsilon_{n}}= & -\frac{1}{\omega_{2}} \int_{D \times \mathcal{H}_{1}(0)} \omega\left(\boldsymbol{x}, \epsilon_{n} \xi\right)|\xi| J(|\xi|) h^{\prime}\left(\epsilon_{n}|\xi|\left|D_{\boldsymbol{e}}^{\epsilon_{n}|\xi|} \boldsymbol{u}^{\epsilon_{n}} \cdot \boldsymbol{e}\right|^{2}\right) \\
& \times 2\left(D_{\boldsymbol{e}}^{\epsilon_{n}|\xi|} \boldsymbol{u}^{\epsilon_{n}} \cdot \boldsymbol{e}\right)\left(D_{\boldsymbol{e}}^{\epsilon_{n}|\xi|} \boldsymbol{w} \cdot \boldsymbol{e}\right) d \xi d \boldsymbol{x}
\end{aligned}
$$

where $\omega(\boldsymbol{x}, \xi)$ is unity if $\boldsymbol{x}+\epsilon_{n} \xi$ is in $D$ and zero otherwise. Note that the boundary component of $\partial D_{\beta}^{+}(\tau)$ given by $\left\{\boldsymbol{x} \in D: \ell(0) \leq x_{1} \leq \ell^{0}(\tau)-\beta, x_{2}=\right.$ $0\}$ is a subset of the failure zone centerline $C^{\epsilon_{n}}(t)$ so for $\boldsymbol{x}$ and $\boldsymbol{y}$ in $F Z^{\epsilon_{n}}(t)$ we see that $\boldsymbol{f}^{\epsilon_{n}}(\boldsymbol{y}, \boldsymbol{x})=0$ or equivalently

$$
h^{\prime}\left(\epsilon_{n}|\xi|\left|D_{\boldsymbol{e}}^{\epsilon_{n}|\xi|} \boldsymbol{u}^{\epsilon_{n}} \cdot \boldsymbol{e}\right|^{2}\right) \times 2\left(D_{\boldsymbol{e}}^{\epsilon_{n}|\xi|} \boldsymbol{u}^{\epsilon_{n}} \cdot \boldsymbol{e}\right)=0
$$

for $\boldsymbol{x}$ and $\boldsymbol{y}=\boldsymbol{x}+\epsilon_{n} \xi$ in $F Z^{\epsilon_{n}}(t)$. Then for for $n$ large enough so that $\ell^{0}(\tau)-\beta<\ell^{\epsilon_{n}}(t)$ and $0<\epsilon_{n}<\beta / 2$ and for test functions $\boldsymbol{w} \in W^{+}\left(D_{\beta}(\tau)\right)$ the product can be written as

$$
\begin{aligned}
h^{\prime} & \left(\epsilon_{n}|\xi|\left|D_{\boldsymbol{e}}^{\epsilon_{n}|\xi|} \boldsymbol{u}^{\epsilon_{n}} \cdot \boldsymbol{e}\right|^{2}\right) \times 2\left(D_{\boldsymbol{e}}^{\epsilon_{n}|\xi|} \boldsymbol{u}^{\epsilon_{n}} \cdot \boldsymbol{e}\right)\left(D_{\boldsymbol{e}}^{\epsilon_{n}|\xi|} \boldsymbol{w} \cdot \boldsymbol{e}\right) \\
& =h^{\prime}\left(\epsilon_{n}\left|\xi \|\left(D_{\boldsymbol{e}}^{\epsilon_{n}|\xi|} \boldsymbol{u}^{\epsilon_{n}} \cdot \boldsymbol{e}\right)^{-}\right|^{2}\right) \times 2\left(\left(D_{\boldsymbol{e}}^{\epsilon_{n}|\xi|} \boldsymbol{u}^{\epsilon_{n}} \cdot \boldsymbol{e}\right)^{-}\right)\left(D_{\boldsymbol{e}}^{\epsilon_{n}|\xi|} \boldsymbol{w} \cdot \boldsymbol{e}\right) \\
& =\chi\left(\boldsymbol{x}, \boldsymbol{x}+\epsilon_{n} \xi\right) h^{\prime}\left(\epsilon_{n}\left|\xi \|\left(D_{\boldsymbol{e}}^{\epsilon_{n}|\xi|} \boldsymbol{u}^{\epsilon_{n}} \cdot \boldsymbol{e}\right)^{-}\right|^{2}\right) \times 2\left(\left(D_{\boldsymbol{e}}^{\epsilon_{n}|\xi|} \boldsymbol{u}^{\epsilon_{n}} \cdot \boldsymbol{e}\right)^{-}\right) \\
& \times\left(D_{\boldsymbol{e}}^{\epsilon_{n}|\xi|} \boldsymbol{w} \cdot \boldsymbol{e}\right),
\end{aligned}
$$

where

$$
\begin{aligned}
& \chi\left(\boldsymbol{x}, \boldsymbol{x}+\epsilon_{n} \xi\right) \\
& \quad= \begin{cases}0, & \text { if the points } \boldsymbol{x}, \boldsymbol{x}+\epsilon_{n} \xi \text { are separated by }\left\{0 \leq x_{1} \leq \ell^{0}(\tau)-\beta, x_{2}=0\right\} \\
1, & \text { otherwise. }\end{cases}
\end{aligned}
$$


(Here we say that $\boldsymbol{x}, \boldsymbol{x}+\epsilon_{n} \xi$ are separated by $\left\{0 \leq x_{1} \leq \ell^{0}(\tau)-\beta, x_{2}=0\right\}$ when it is impossible to connect these two points by a line segment without crossing $\left\{0 \leq x_{1} \leq \ell^{0}(\tau)-\beta, x_{2}=0\right\}$.) Then $I^{\epsilon_{n}}$ becomes

$$
\begin{aligned}
& I^{\epsilon_{n}}=-\frac{1}{\omega_{2}} \int_{D \times \mathcal{H}_{1}(0)} \omega\left(\boldsymbol{x}, \epsilon_{n} \xi\right) \chi\left(\boldsymbol{x}, \boldsymbol{x}+\epsilon_{n} \xi\right)|\xi| J(|\xi|) h^{\prime}\left(\epsilon_{n}|\xi|\left|\left(D_{\boldsymbol{e}}^{\epsilon_{n}|\xi|} \boldsymbol{u}^{\epsilon_{n}} \cdot \boldsymbol{e}\right)^{-}\right|^{2}\right) \\
& \times 2\left(\left(D_{\boldsymbol{e}}^{\epsilon_{n}|\xi|} \boldsymbol{u}^{\epsilon_{n}} \cdot \boldsymbol{e}\right)^{-}\right)\left(D_{\boldsymbol{e}}^{\epsilon_{n}|\xi|} \boldsymbol{w} \cdot \boldsymbol{e}\right) d \xi d \boldsymbol{x} .
\end{aligned}
$$

We can now bound (6.66) as in (6.37) and change the order of integration to arrive at the upper bound

$$
\left|I^{\epsilon_{n}}\right| \leq \frac{2 h^{\prime}(0) C}{\omega_{2}}\left(\int_{\mathcal{H}_{1}(0)} \int_{D} \omega\left(\boldsymbol{x}, \epsilon_{n} \xi\right) \chi\left(\boldsymbol{x}, \boldsymbol{x}+\epsilon_{n} \xi\right)\left|D_{e}^{\epsilon_{n}|\xi|} \boldsymbol{w} \cdot \boldsymbol{e}\right|^{2} d \boldsymbol{x} d \xi\right)^{1 / 2} .
$$

We change to slicing variables and write $\boldsymbol{x}=\boldsymbol{y}+r \boldsymbol{e}$, where $\boldsymbol{e}$ is on the unit circle and $\boldsymbol{y} \in \Pi_{\boldsymbol{e}}$ where $\Pi_{\boldsymbol{e}}$ is the subspace perpendicular to $\boldsymbol{e}$ and $r \in \mathbb{R}$. We set $D_{\boldsymbol{y}}^{e}=\left\{r \in \mathbb{R}: \boldsymbol{y}+r \boldsymbol{e} \in D_{\beta}(\tau)\right\}$ and $D^{e}=\left\{\boldsymbol{y} \in \Pi_{\boldsymbol{e}}: D_{\boldsymbol{y}}^{e} \neq \emptyset\right\}$ so

$$
\left|I^{\epsilon_{n}}\right| \leq \frac{2 h^{\prime}(0) C}{\omega_{2}}\left(\int_{\mathcal{H}_{1}(0)} \int_{D^{e}} \int_{D_{\boldsymbol{y}}^{e}} \chi\left(\boldsymbol{y}+r \boldsymbol{e}, \boldsymbol{y}+\left(r+\epsilon_{n}|\xi|\right) \boldsymbol{e}\right)\left|D_{e}^{\epsilon_{n}|\xi|} \boldsymbol{w} \cdot \boldsymbol{e}\right|^{2} d r d \boldsymbol{y} d \xi\right)^{1 / 2} .
$$

We use the fact that functions in Sobolev spaces are absolutely continuous for a.e. lines to write $(6.38)$ for $\boldsymbol{w} \in W^{+}\left(D_{\beta}(\tau)\right)$ and

$$
\begin{aligned}
\left|I^{\epsilon_{n}}\right| & \frac{2 h^{\prime}(0) C}{\omega_{2}}\left(\int_{\mathcal{H}_{1}(0)} \int_{D^{e}} \int_{D_{y}^{e}} \chi\left(\boldsymbol{y}+r \boldsymbol{e}, \boldsymbol{y}+\left(r+\epsilon_{n}|\xi|\right) \boldsymbol{e}\right) \mid\right. \\
& \left.\left.\int_{0}^{1} \mathcal{E} \boldsymbol{w}\left(\boldsymbol{y}+\left(r+s \epsilon_{n}|\xi|\right) \boldsymbol{e}\right) \boldsymbol{e} \cdot \boldsymbol{e} d s\right|^{2} d r d \boldsymbol{y} d \xi\right)^{1 / 2} \\
\leq & \frac{2 h^{\prime}(0) C}{\omega_{2}}\left(\int_{\mathcal{H}_{1}(0)} \int_{0}^{1} \int_{D^{e}} \int_{D_{y}^{e}}\right. \\
& \left.\chi\left(\boldsymbol{y}+r \boldsymbol{e}, \boldsymbol{y}+\left(r+\epsilon_{n}|\xi|\right) \boldsymbol{e}\right)\left|\mathcal{E} \boldsymbol{w}\left(\boldsymbol{y}+\left(r+s \epsilon_{n}|\xi|\right) \boldsymbol{e}\right) \boldsymbol{e} \cdot \boldsymbol{e}\right|^{2} d r d \boldsymbol{y} d s d \xi\right)^{1 / 2} .
\end{aligned}
$$

where Jensen inequality and Fubini's theorem have been applied in the last line. Introducing $\chi_{D_{\beta}(\tau)}(\boldsymbol{x})=1$ if its argument lies in $D_{\beta}(\tau)$ and zero otherwise, applying $\chi\left(\boldsymbol{y}+r \boldsymbol{e}, \boldsymbol{y}+\left(r+\epsilon_{n}|\xi|\right) \boldsymbol{e}\right) \leq \chi_{D_{\beta}(\tau)}(\boldsymbol{y}+r \boldsymbol{e}) \chi_{D_{\beta}(\tau)}\left(\boldsymbol{y}+\left(r+s \epsilon_{n}|\xi|\right) \boldsymbol{e}\right)$ and changing to original variables gives

$$
\begin{aligned}
\left|I^{\epsilon_{n}}\right| \leq & \frac{2 h^{\prime}(0) C}{\omega_{2}}\left(\int_{0}^{1} \int_{\mathcal{H}_{1}(0)} \int_{D} \chi_{D_{\beta}(\tau)}(\boldsymbol{x}) \chi_{D_{\beta}(\tau)}\right. \\
& \left.\left(\boldsymbol{x}+s \epsilon_{n}|\xi| \boldsymbol{e}\right)\left|\mathcal{E} \boldsymbol{w}\left(\boldsymbol{x}+s \epsilon_{n}|\xi| \boldsymbol{e}\right) \boldsymbol{e} \cdot \boldsymbol{e}\right|^{2} d \boldsymbol{x} d \xi d s\right)^{1 / 2} .
\end{aligned}
$$

From this we conclude

$$
\left|I^{\epsilon_{n}}\right| \leq C\|\boldsymbol{w}\|_{H^{1}\left(D_{\beta}(\tau) ; \mathbb{R}^{2}\right)} .
$$


Arguments identical to the proof of Lemma 3.1 show that the sequence $\boldsymbol{b}^{\epsilon_{n}}$ is uniformly bounded in $W^{+}\left(D_{\beta}(\tau)\right)^{\prime}$ for all $\tau \in[0, T]$ and $\epsilon_{n}>0$ and together with (6.71) one concludes

$$
\sup _{t \in(\tau, T)}\left\|\ddot{\boldsymbol{u}}^{\epsilon_{n}}(t)\right\|_{W^{+}\left(D_{\beta}(\tau) ; \mathbb{R}^{2}\right)^{\prime}}<C, \text { for } \beta / 2>\epsilon_{n}>0 .
$$

Hence

$$
\int_{\tau}^{T}\left\|\ddot{\boldsymbol{u}}^{\epsilon_{n}}(t)\right\|_{W^{+}\left(D_{\beta}(\tau) ; \mathbb{R}^{2}\right)^{\prime}}^{2} d t<\infty \text { for } \beta / 2>\epsilon_{n}>0,
$$

and passing to a subsequence if necessary gives a $\boldsymbol{v}(t)$ in $L^{2}\left(\tau, T ; W^{+}\left(D_{\beta}(\tau)\right)^{\prime}\right)$ such that $\ddot{\boldsymbol{u}}^{\epsilon_{n}} \rightarrow \boldsymbol{v}$ weakly in $L^{2}\left(\tau, T ; W^{+}\left(D_{\beta}(\tau)^{\prime}\right)\right.$.

We finish the proof by showing $\boldsymbol{v}=\ddot{\boldsymbol{u}}_{\tau}^{0}$. To see this note $\boldsymbol{u}^{\epsilon_{n}} \in C^{2}([0, T]$; $\left.L^{2}\left(D, \mathbb{R}^{2}\right)\right)$ and for $\varphi \in C_{c}^{\infty}(\tau, T)$ and for $\boldsymbol{w} \in W^{+}\left(D_{\beta}(\tau)\right)$ we have

$$
\int_{\tau}^{T} \int_{D} \ddot{\boldsymbol{u}}^{\epsilon_{n}} \cdot \boldsymbol{w} d \boldsymbol{x} \varphi(t) d t=-\int_{\tau}^{T} \int_{D} \dot{\boldsymbol{u}}^{\epsilon_{n}} \cdot \boldsymbol{w} d \boldsymbol{x} \dot{\varphi}(t) d t
$$

Passing to the $\epsilon_{n}=0$ limit using Lemma 3.2 applied to the right hand side gives

$$
\int_{\tau}^{T}\langle\boldsymbol{v}, \boldsymbol{w}\rangle \varphi(t) d t=-\int_{\tau}^{T} \int_{D} \dot{\boldsymbol{u}}^{0} \cdot \boldsymbol{w} \dot{\varphi}(t) d \boldsymbol{x} d t, \text { for all } \boldsymbol{w} \in W^{+}\left(D_{\beta}(\tau)\right)
$$

and we deduce from (6.75) that $\boldsymbol{v}=\ddot{\boldsymbol{u}}_{\tau}^{0}$ as elements of $W^{+}\left(D_{\beta}(\tau)\right)^{\prime}$. Identical arguments show that $\ddot{\boldsymbol{u}}_{\tau}^{0} \in W^{-}\left(D_{\beta}(\tau)\right)^{\prime}$ and Theorem 3.2 is proved.

We now prove Lemma 6.3. We illustrate the proof for $\boldsymbol{w}(\boldsymbol{x}) \in W^{+}\left(D_{\beta}(\tau)\right)$ noting an identical proof holds for $\boldsymbol{w} \in W^{-}\left(D_{\beta}(\tau)\right)$. Multiply both sides of (6.61) by $\varphi(t) \in C_{c}^{\infty}(\tau, T)$ and integrate with respect to $t$ over $(\tau, T)$ to obtain

$$
\begin{aligned}
\int_{\tau}^{T} & \rho \int_{D} \ddot{\boldsymbol{u}}^{\epsilon_{n}}(\boldsymbol{x}, t) \cdot \boldsymbol{w}(\boldsymbol{x}) d \boldsymbol{x} \varphi(t) d t \\
= & -\int_{\tau}^{T} \int_{D} \int_{\mathcal{H}_{\epsilon_{n}}(\boldsymbol{x}) \cap D}|\boldsymbol{y}-\boldsymbol{x}| \partial_{S} \mathcal{W}^{\epsilon_{n}}\left(\boldsymbol{y}-\boldsymbol{x}, S\left(\boldsymbol{y}, \boldsymbol{x}, \boldsymbol{u}^{\epsilon_{n}}(t)\right)\right) S(\boldsymbol{y}, \boldsymbol{x}, \boldsymbol{w}) \\
& \boldsymbol{y} d \boldsymbol{x} \varphi(t) d t \\
& +\int_{\tau}^{T} \int_{D} \boldsymbol{b}^{\epsilon_{n}}(\boldsymbol{x}, t) \cdot \boldsymbol{w}(\boldsymbol{x}) d \boldsymbol{x} \varphi(t) d t
\end{aligned}
$$

The goal is to pass to the $\epsilon_{n}=0$ limit in this equation to recover (6.3). The limit of the left hand side of (6.76) follows from Theorem 3.2

$$
\lim _{\epsilon_{n} \rightarrow 0} \int_{\tau}^{T} \varphi(t) \rho \int_{D} \ddot{\boldsymbol{u}}^{\epsilon_{n}}(\boldsymbol{x}, t) \cdot \boldsymbol{w}(\boldsymbol{x}) d \boldsymbol{x} d t=\int_{\tau}^{T} \varphi(t) \rho\left\langle\ddot{\boldsymbol{u}}_{\tau}^{0}(t), \boldsymbol{w}\right\rangle d t .
$$

The first term on the right hand side of (6.76) is written

$$
\int_{\tau}^{T} \varphi I^{\epsilon_{n}} d t
$$

We can recover the $\epsilon_{n}=0$ limit of the first term on the right hand side of (6.76) by appealing to the bound (6.71) to pass to the limit under the time integral using Lebesgue dominated convergence once we show that for every 
$\boldsymbol{w} \in W^{+}\left(D_{\beta}(\tau)\right)$ the bounded sequence $\left\{I^{\epsilon_{n}}(t)\right\}$ has a limit for a.e. $t \in(\tau, T)$. To see this we apply (6.64) to get that

$$
\begin{aligned}
& I^{\epsilon_{n}}(t)=-\int_{D} \int_{\mathcal{H}_{\epsilon_{n}}(\boldsymbol{x}) \cap D}|\boldsymbol{y}-\boldsymbol{x}| \partial_{S} \mathcal{W}^{\epsilon_{n}} \\
& \times\left(\boldsymbol{y}-\boldsymbol{x}, S\left(\boldsymbol{y}, \boldsymbol{x}, \boldsymbol{u}^{\epsilon_{n}}(t)\right)\right) S(\boldsymbol{y}, \boldsymbol{x}, \boldsymbol{w}) d \boldsymbol{y} d \boldsymbol{x} \\
&=-\frac{1}{\omega_{2}} \int_{D \times \mathcal{H}_{1}(0)} \omega\left(\boldsymbol{x}, \epsilon_{n} \xi\right) \chi\left(\boldsymbol{x}, \boldsymbol{x}+\epsilon_{n} \xi\right)|\xi| J(|\xi|) h^{\prime}\left(\epsilon_{n}|\xi|\left|\left(D_{e}^{\epsilon_{n}|\xi|} \boldsymbol{u}^{\epsilon_{n}} \cdot \boldsymbol{e}\right)^{-}\right|^{2}\right) \\
& \quad \times 2\left(\left(D_{\boldsymbol{e}}^{\epsilon_{n}|\xi|} \boldsymbol{u}^{\epsilon_{n}} \cdot \boldsymbol{e}\right)^{-}\right)\left(D_{\boldsymbol{e}}^{\epsilon_{n}|\xi|} \boldsymbol{w} \cdot \boldsymbol{e}\right) d \xi d \boldsymbol{x} .
\end{aligned}
$$

The integrand is the product of two factors (note $\omega\left(\boldsymbol{x}, \epsilon_{n} \xi\right) \chi\left(\boldsymbol{x}, \boldsymbol{x}+\epsilon_{n} \xi\right)=$ $\left.\omega\left(\boldsymbol{x}, \epsilon_{n} \xi\right)^{2} \chi\left(\boldsymbol{x}, \boldsymbol{x}+\epsilon_{n} \xi\right)^{2}\right)$ and we show that on passing to a subsequence if necessary the first factor

$$
\begin{aligned}
& \omega\left(\boldsymbol{x}, \epsilon_{n} \xi\right) \chi\left(\boldsymbol{x}, \boldsymbol{x}+\epsilon_{n} \xi\right) h^{\prime}\left(\epsilon_{n}\left|\xi \|\left(D_{\boldsymbol{e}}^{\epsilon_{n}|\xi|} \boldsymbol{u}^{\epsilon_{n}} \cdot \boldsymbol{e}\right)^{-}\right|^{2}\right) \times 2\left(\left(D_{\boldsymbol{e}}^{\epsilon_{n}|\xi|} \boldsymbol{u}^{\epsilon_{n}} \cdot \boldsymbol{e}\right)^{-}\right) \\
& \left.\quad \rightarrow 2 h^{\prime}(0) g(\boldsymbol{x}, \xi, t)\right)
\end{aligned}
$$

weakly in $L^{2}\left(D \times \mathcal{H}_{1}(0), \mathbb{R}\right)$ and the second factor

$$
\omega\left(\boldsymbol{x}, \epsilon_{n} \xi\right) \chi\left(\boldsymbol{x}, \boldsymbol{x}+\epsilon_{n} \xi\right) D_{\boldsymbol{e}}^{\epsilon_{n}|\xi|} \boldsymbol{w} \cdot \boldsymbol{e} \rightarrow \mathcal{E} \boldsymbol{w}(\boldsymbol{x}) \boldsymbol{e} \cdot \boldsymbol{e} .
$$

strong in $L^{2}\left(D \times \mathcal{H}_{1}(0), \mathbb{R}\right)$. Here as in Sect. 5 the $L^{2}$ norm and inner product are with respect to the weighted measure $|\xi| J(|\xi|) d \xi d \boldsymbol{x}$. Hence for fixed $t$ we suppose that (6.80) and (6.81) hold to conclude that for any cluster point of $\left\{I^{\epsilon_{n}}(t)\right\}$ there is a subsequence

$$
\begin{aligned}
\left.\lim _{\epsilon_{n^{\prime}} \rightarrow 0} I^{\epsilon_{n^{\prime}}}(t)=-\int_{D} \int_{\mathcal{H}_{1}(0)}|\xi| J(|\xi|) 2 h^{\prime}(0) g(\boldsymbol{x}, \xi, t)\right) \mathcal{E} \boldsymbol{w}(\boldsymbol{x}) \boldsymbol{e} \cdot \boldsymbol{e} d \xi d \boldsymbol{x} \\
=-\int_{D} \int_{\mathcal{H}_{1}(0)} 2|\xi| J(|\xi|) h^{\prime}(0)\left(\mathcal{E} \boldsymbol{u}^{0}(t, \boldsymbol{x}) \boldsymbol{e} \cdot \boldsymbol{e}\right)(\mathcal{E} \boldsymbol{w}(\boldsymbol{x}) \boldsymbol{e} \cdot \boldsymbol{e}) d \xi d \boldsymbol{x} \\
=-\int_{D} \mathbb{C} \mathcal{E} \boldsymbol{u}^{0}(t, \boldsymbol{x}): \mathcal{E} \boldsymbol{w}(\boldsymbol{x}) d \boldsymbol{x},
\end{aligned}
$$

where the second line follows from (5.20) and the third line follows from a straightforward calculation, see, e.g., [24]. One obtains the same limit for subsequences of all possibly distinct cluster points of $\left\{I^{\epsilon_{n}}(t)\right\}$ to conclude there is one cluster point and we have identified $\lim _{\epsilon_{n} \rightarrow 0} I^{\epsilon_{n}}(t)$ for a.e. $t \in(0, T)$.

To conclude the weak and strong convergences (6.80) and (6.81) are established. First note that $h^{\prime}(r)$ is monotone decreasing in $r$ so $h^{\prime}\left(\epsilon_{n}|\xi| \mid\left(D_{e}^{\epsilon_{n}|\xi|}\right.\right.$ $\left.\left.\boldsymbol{u}^{\epsilon_{n}} \cdot \boldsymbol{e}\right)\left.^{-}\right|^{2}\right) \leq h^{\prime}(0)$ and from (5.18) we have $\left(D_{\boldsymbol{e}}^{\epsilon_{n}|\xi|} \boldsymbol{u}^{\epsilon_{n}} \cdot \boldsymbol{e}\right)^{-}$is bounded in $L^{2}\left(D \times \mathcal{H}_{1}(0), \mathbb{R}\right)$ so the first factor is bounded in $L^{2}\left(D \times \mathcal{H}_{1}(0), \mathbb{R}\right)$ uniformly in $\epsilon_{n}$ and has a subsequence that converges weakly to a limit written $K(\boldsymbol{x}, \xi, t)$. Application of Lemma 6.5 of [24] and (5.19) allows us to identify $\left.K(\boldsymbol{x}, \xi(t))=2 h^{\prime}(0) g(\boldsymbol{x}, \xi, t)\right)$ where we have explicitly written the time dependence of $g(\boldsymbol{x}, \xi)$ and weak convergence is established. To show the strong 
convergence (6.81) we form

$$
\begin{aligned}
A^{\epsilon_{n}} & =\frac{1}{\omega_{2}} \int_{D \times \mathcal{H}_{1}(0)} \omega\left(\boldsymbol{x}, \epsilon_{n} \xi\right) \chi\left(\boldsymbol{x}, \boldsymbol{x}+\epsilon_{n} \xi\right)|\xi| J(|\xi|) \\
& \times\left|\left(D_{\boldsymbol{e}}^{\epsilon_{n}|\xi|} \boldsymbol{w} \cdot \boldsymbol{e}\right)-\mathcal{E} \boldsymbol{w}(\boldsymbol{x}) \boldsymbol{e} \cdot \boldsymbol{e}\right|^{2} d \xi d \boldsymbol{x} .
\end{aligned}
$$

Estimating as in (6.67) - (6.70) we get

$$
\begin{aligned}
\lim _{\epsilon_{n} \rightarrow 0} & A^{\epsilon_{n}} \\
\leq & \lim _{\epsilon_{n} \rightarrow 0} \int_{0}^{1} \frac{1}{\omega_{2}} \int_{D \times \mathcal{H}_{1}(0)} \chi_{D_{\beta}(\tau)}(\boldsymbol{x}) \chi_{D_{\beta}(\tau)}\left(\boldsymbol{x}+s \epsilon_{n} \xi\right)|\xi| J(|\xi|) \\
& \left|\mathcal{E} \boldsymbol{w}\left(\boldsymbol{x}+s \epsilon_{n}|\xi| \boldsymbol{e}\right)-\mathcal{E} \boldsymbol{w}(\boldsymbol{x}) \boldsymbol{e} \cdot \boldsymbol{e}\right|^{2} d \xi d \boldsymbol{x} d s \\
= & \int_{0}^{1} \int_{D_{\beta}(\tau)} s^{2} \lim _{\epsilon_{n} \rightarrow 0} \frac{1}{s^{2} \omega_{2}} \int_{\mathcal{H}_{1}(0)} \chi_{D_{\beta}(\tau)}\left(\boldsymbol{x}+s \epsilon_{n} \xi\right)|\xi| J(|\xi|) \\
& \left|\mathcal{E} \boldsymbol{w}\left(\boldsymbol{x}+s \epsilon_{n}|\xi| \boldsymbol{e}\right)-\mathcal{E} \boldsymbol{w}(\boldsymbol{x}) \boldsymbol{e} \cdot \boldsymbol{e}\right|^{2} d \xi d \boldsymbol{x} d s \\
= & 0
\end{aligned}
$$

where we use Lebesgue dominated convergence to interchange limit and integral noting that the point wise limit is 0 and holds a.e. $\boldsymbol{x} \in D_{\beta}(\tau)$ at the Lebesgue points

$$
\lim _{\epsilon_{n} \rightarrow 0} \frac{1}{s^{2} \omega_{2}} \int_{\mathcal{H}_{1}(0)}|\xi| J(|\xi|)\left|\mathcal{E} \boldsymbol{w}\left(\boldsymbol{x}+s \epsilon_{n}|\xi| \boldsymbol{e}\right)-\mathcal{E} \boldsymbol{w}(\boldsymbol{x}) \boldsymbol{e} \cdot \boldsymbol{e}\right|^{2} d \xi=0
$$

This establishes strong convergence for $\boldsymbol{w} \in W^{+}\left(D_{\beta}(\tau)\right)$. Collecting results gives that the limit of the first term on the right hand side of (6.61) is

$$
\lim _{\epsilon_{n} \rightarrow 0} \int_{0}^{T} \varphi(t) I^{\epsilon_{n}} d t=-\int_{0}^{T} \varphi(t) \int_{D} \mathbb{C} \mathcal{E} \boldsymbol{u}^{0}: \mathcal{E} \boldsymbol{w} d \boldsymbol{x} d t .
$$

Passing to the limit on the last term of the right hand side of (6.61) and arguments similar to before give

$$
\lim _{\epsilon_{n} \rightarrow 0} \int_{\tau}^{T} \int_{D} \boldsymbol{b}^{\epsilon_{n}} \cdot \boldsymbol{w} d \boldsymbol{x} \varphi(t) d t=\int_{\tau}^{T} \int_{\partial D} \boldsymbol{g} \cdot \boldsymbol{w} d \sigma \varphi(t) d t .
$$

and we conclude that

$$
\begin{aligned}
\int_{\tau}^{T} \varphi(t) \rho\left\langle\ddot{\boldsymbol{u}}^{0}(t), \boldsymbol{w}\right\rangle d t= & -\int_{\tau}^{T} \varphi(t)\left(\int_{D} \mathbb{C} \mathcal{E} \boldsymbol{u}^{0}: \mathcal{E} \boldsymbol{w} d x\right. \\
& \left.+\int_{\partial D} \boldsymbol{g} \cdot \boldsymbol{w} d \sigma\right) d t
\end{aligned}
$$

for all $\boldsymbol{w} \in W^{+}\left(D_{\beta}(\tau)\right)$ and Lemma 6.3 is proved.

\section{Weak solution of the wave equation on $D_{t}$}

Theorem 3.4 is proved in this section. From Theorem 3.1 and Lemma 3.2 the limit displacement $\boldsymbol{u}^{0}$ belongs to $\mathcal{V}$. From Lemma 2.8 and Remark 2.9 of [12] we have that if $\boldsymbol{u} \in \mathcal{V}$ and (3.19) holds for every $\varphi \in C_{c}^{\infty}\left((0, T) ; V_{T}\right)$ with 
$\varphi(t) \in V_{t}$ then $\boldsymbol{u}$ is a weak solution of (3.18). Motivated by this we begin by selecting a class of trial fields that are convenient to work with. For $t \in[0, T]$ set $s_{\beta}(t)=t-\beta$ for some fixed number $\beta \in(0, t)$. Given $\boldsymbol{w} \in C_{c}^{\infty}\left((0, T) ; V_{T}\right)$ with $\boldsymbol{w} \in V_{t}$ for $t \in(0, T)$, set $\tilde{\boldsymbol{w}}(t)=\boldsymbol{w}\left(s_{\beta}(t)\right) \in V_{s_{\beta}(t)} \subset V_{t}$. Substitution of this trial in (2.15) gives the identity

$$
\begin{aligned}
& \rho \int_{0}^{T} \int_{D} \dot{\boldsymbol{u}}^{\epsilon_{n}}(t) \cdot \dot{\tilde{\boldsymbol{w}}}(t) d \boldsymbol{x} d t \\
& \quad=\int_{0}^{T} \int_{D} \int_{\mathcal{H}_{\epsilon_{n}}(\boldsymbol{x}) \cap D}|\boldsymbol{y}-\boldsymbol{x}| \partial_{S} \mathcal{W}^{\epsilon_{n}}(\boldsymbol{y}-\boldsymbol{x}, \\
& \left.\quad S\left(\boldsymbol{y}, \boldsymbol{x}, \boldsymbol{u}^{\epsilon_{n}}(t)\right)\right) S(\boldsymbol{y}, \boldsymbol{x}, \tilde{\boldsymbol{w}}(t)) d \boldsymbol{y} d \boldsymbol{x} d t \\
& \quad-\int_{0}^{T} \int_{D} \boldsymbol{b}^{\epsilon_{n}}(t) \cdot \tilde{\boldsymbol{w}}(t) d \boldsymbol{x} d t, \text { for } \epsilon_{n}>0 .
\end{aligned}
$$

Here we will pass to the $\epsilon_{n}=0$ limit in this identity to obtain an $\epsilon_{n}=0$ identity. Then on passing to the $\beta \rightarrow 0$ limit in each term we will show that $\boldsymbol{u}^{0}$ is a weak solution. We begin by understanding the limit of the middle term in (7.1) for a given sequence indexed by $\epsilon_{n}$. We write out the integrand appearing under the time integral

$$
\begin{aligned}
& I^{\epsilon_{n}}(t, \tilde{\boldsymbol{w}}(t))=\int_{D} \int_{\mathcal{H}_{\epsilon_{n}}(x) \cap D}|\boldsymbol{y}-\boldsymbol{x}| \partial_{S} \mathcal{W}^{\epsilon_{n}} \\
& \quad \times\left(\boldsymbol{y}-\boldsymbol{x}, S\left(\boldsymbol{y}, \boldsymbol{x}, \boldsymbol{u}^{\epsilon_{n}}(t)\right)\right) S(\boldsymbol{y}, \boldsymbol{x}, \tilde{\boldsymbol{w}}(t)) d \boldsymbol{y} d \boldsymbol{x}
\end{aligned}
$$

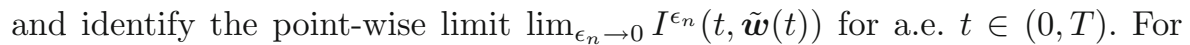
this choice of test function we change variables as in (6.33) to obtain

$$
I^{\epsilon_{n}}(t, \tilde{\boldsymbol{w}})=I_{1}^{\epsilon_{n}}(t, \tilde{\boldsymbol{w}})+I_{2}^{\epsilon_{n}}(t, \tilde{\boldsymbol{w}}),
$$

where

$$
\begin{aligned}
I_{1}^{\epsilon_{n}}(t, \tilde{\boldsymbol{w}})= & \frac{1}{\omega_{2}} \int_{D \times \mathcal{H}_{1}(0)} \omega\left(\boldsymbol{x}, \epsilon_{n} \xi\right)|\xi| J(|\xi|) h^{\prime}\left(\epsilon_{n}|\xi|\left|\left(D_{\boldsymbol{e}}^{\epsilon_{n}|\xi|} \boldsymbol{u}^{\epsilon_{n}} \cdot \boldsymbol{e}\right)^{-}\right|^{2}\right) \\
& \times 2\left(\left(D_{\boldsymbol{e}}^{\epsilon_{n}|\xi|} \boldsymbol{u}^{\epsilon_{n}} \cdot \boldsymbol{e}\right)^{-}\right)\left(D_{\boldsymbol{e}}^{\epsilon_{n}|\xi|} \tilde{\boldsymbol{w}} \cdot \boldsymbol{e}\right) d \xi d \boldsymbol{x}, \\
I_{2}^{\epsilon_{n}}(t, \tilde{\boldsymbol{w}})= & \frac{1}{\omega_{2}} \int_{D \times \mathcal{H}_{1}(0) \cap\left\{S Z^{\epsilon_{n}}(t) \backslash F Z_{n}^{\epsilon_{n}}(t)\right\} \cap A_{\epsilon_{n}}^{+}} \omega\left(\boldsymbol{x}, \epsilon_{n} \xi\right)|\xi| J(|\xi|) h^{\prime} \\
& \times\left(\epsilon_{n}|\xi|\left|D_{\boldsymbol{e}}^{\epsilon_{n}|\xi|} \boldsymbol{u}^{\epsilon_{n}} \cdot \boldsymbol{e}\right|^{2}\right) \\
& \times 2\left(D_{\boldsymbol{e}}^{\epsilon_{n}|\xi|} \boldsymbol{u}^{\epsilon_{n}} \cdot \boldsymbol{e}\right)\left(D_{\boldsymbol{e}}^{\epsilon_{n}|\xi|} \tilde{\boldsymbol{w}} \cdot \boldsymbol{e}\right) d \xi d \boldsymbol{x} .
\end{aligned}
$$

As in (6.66) we have

$$
\begin{aligned}
& I_{1}^{\epsilon_{n}}(t, \tilde{\boldsymbol{w}})=\frac{1}{\omega_{2}} \int_{D \times \mathcal{H}_{1}(0)} \omega\left(\boldsymbol{x}, \epsilon_{n} \xi\right) \tilde{\chi}\left(\boldsymbol{x}, \boldsymbol{x}+\epsilon_{n} \xi\right)|\xi| J(|\xi|) h^{\prime} \\
& \quad \times\left(\epsilon_{n}|\xi|\left|\left(D_{\boldsymbol{e}}^{\epsilon_{n}|\xi|} \boldsymbol{u}^{\epsilon_{n}} \cdot \boldsymbol{e}\right)^{-}\right|^{2}\right) \\
& \quad \times 2\left(\left(D_{\boldsymbol{e}}^{\epsilon_{n}|\xi|} \boldsymbol{u}^{\epsilon_{n}} \cdot \boldsymbol{e}\right)^{-}\right)\left(D_{\boldsymbol{e}}^{\epsilon_{n}|\xi|} \tilde{\boldsymbol{w}} \cdot \boldsymbol{e}\right) d \xi d \boldsymbol{x}
\end{aligned}
$$


where

$\tilde{\chi}\left(\boldsymbol{x}, \boldsymbol{x}+\epsilon_{n} \xi\right)$

$=\left\{\begin{array}{l}0, \quad \text { if the points } \boldsymbol{x}, \boldsymbol{x}+\epsilon_{n} \xi \text { are separated by }\left\{0 \leq x_{1} \leq \ell^{0}(t-\beta), x_{2}=0\right\} \\ 1, \quad \text { otherwise, }\end{array}\right.$

for $n$ large enough so that $\ell^{0}(\beta-t)<\ell^{\epsilon_{n}}(t)$ and $0<\epsilon_{n}<\left(\ell^{0}(t)-\ell^{0}(t-\beta)\right) / 2$, where $\beta \in(0, t)$. (Here we have used that $\ell^{0}(t)$ is continuous and strictly increasing.) As in the proof of Lemma 6.3 the integrand is the product of two factors such that the first factor

$$
\begin{aligned}
& \omega\left(\boldsymbol{x}, \epsilon_{n} \xi\right) \tilde{\chi}\left(\boldsymbol{x}, \boldsymbol{x}+\epsilon_{n} \xi\right) h^{\prime} \\
& \left.\quad \times\left(\epsilon_{n}|\xi|\left|\left(D_{\boldsymbol{e}}^{\epsilon_{n}|\xi|} \boldsymbol{u}^{\epsilon_{n}} \cdot \boldsymbol{e}\right)^{-}\right|^{2}\right) \times 2\left(\left(D_{\boldsymbol{e}}^{\epsilon_{n}|\xi|} \boldsymbol{u}^{\epsilon_{n}} \cdot \boldsymbol{e}\right)^{-}\right) \rightarrow 2 h^{\prime}(0) g(\boldsymbol{x}, \xi, t)\right)
\end{aligned}
$$

weakly in $L^{2}\left(D \times \mathcal{H}_{1}(0), \mathbb{R}\right)$ and the second factor

$$
\omega\left(\boldsymbol{x}, \epsilon_{n} \xi\right) \tilde{\chi}\left(\boldsymbol{x}, \boldsymbol{x}+\epsilon_{n} \xi\right) D_{\boldsymbol{e}}^{\epsilon_{n}|\xi|} \tilde{\boldsymbol{w}} \cdot \boldsymbol{e} \rightarrow \mathcal{E} \tilde{\boldsymbol{w}}(\boldsymbol{x}) \boldsymbol{e} \cdot \boldsymbol{e}
$$

strong in $L^{2}\left(D \times \mathcal{H}_{1}(0), \mathbb{R}\right)$. Here $g(\boldsymbol{x}, \xi, t)$ is the weak limit given by (5.19) with the time dependence explicitly written. Hence we conclude using the same arguments given in the proof of Lemma 6.3 that

$$
\lim _{\epsilon_{n} \rightarrow 0} I_{1}^{\epsilon_{n}}(t, \tilde{\boldsymbol{w}})=\int_{D} \mathbb{C} \mathcal{E} \boldsymbol{u}^{0}(t): \mathcal{E} \tilde{\boldsymbol{w}} d \boldsymbol{x} .
$$

From hypothesis 3.1, noting that $\ell^{0}(t)$ is strictly increasing and continuous, we have that $\left|\left\{S Z^{\epsilon_{n}} \backslash F Z^{\epsilon_{n}}\right\}\right| \leq C\left|\epsilon_{n}\right|^{2}$. We estimate $I_{2}^{\epsilon_{n}}(t, \tilde{\boldsymbol{w}})$ recalling (6.34) and (6.41) to obtain

$$
\begin{aligned}
\left|I_{2}^{\epsilon_{n}}(t, \tilde{\boldsymbol{w}})\right| & \\
\leq & \frac{1}{\rho \omega_{2}} \int_{D \times \mathcal{H}_{1}(0) \cap\left\{S Z^{\epsilon_{n}}(t) \backslash F Z^{\epsilon_{n}}(t)\right\} \cap A_{\epsilon_{n}}^{+}} \\
& \omega\left(\boldsymbol{x}, \epsilon_{n} \xi\right)|\xi| J(|\xi|) \frac{2 h^{\prime}\left(\left(r^{c}\right)^{2}\right) r^{c}}{\sqrt{\epsilon_{n}|\xi|}}\left|D_{e}^{\epsilon_{n}|\xi|} \tilde{\boldsymbol{w}} \cdot \boldsymbol{e}\right| d \xi d \boldsymbol{x}, \\
\leq & \frac{1}{\rho \omega_{2}}\left(\int_{D \times \mathcal{H}_{1}(0) \cap\left\{S Z^{\epsilon_{n}}(t) \backslash F Z^{\epsilon_{n}}(t)\right\}} \omega\left(\boldsymbol{x}, \epsilon_{n} \xi\right)|\xi| J(|\xi|) \frac{\left(2 h^{\prime}\left(\left(r^{c}\right)^{2}\right) r^{c}\right)^{2}}{\epsilon_{n}|\xi|} d \xi d \boldsymbol{x}\right)^{1 / 2} \times \\
& \left(\int_{D \times \mathcal{H}_{1}(0) \cap\left\{S Z^{\epsilon_{n}}(t) \backslash F Z^{\epsilon_{n}}(t)\right\}} \omega\left(\boldsymbol{x}, \epsilon_{n} \xi\right)|\xi| J(|\xi|)\left|D_{e}^{\epsilon_{n}|\xi|} \tilde{\boldsymbol{w}} \cdot \boldsymbol{e}\right|^{2} d \xi d \boldsymbol{x} d t\right)^{1 / 2} \\
\leq & C \sqrt{\left|\epsilon_{n}\right|}\|\tilde{\boldsymbol{w}}\|_{H^{1}\left(D_{\beta}(t) ; \mathbb{R}^{2}\right) .}
\end{aligned}
$$

Here the last inequality is obtained using slicing variables noting that $\tilde{\boldsymbol{w}} \in$ $H^{1}\left(D_{\beta}(t)\right)$. From this we conclude that $\lim _{\epsilon_{n} \rightarrow 0} I^{\epsilon_{n}}(t, \tilde{\boldsymbol{w}})$ exists and

$$
\lim _{\epsilon_{n} \rightarrow 0} I^{\epsilon_{n}}(t, \tilde{\boldsymbol{w}})=\int_{D} \mathbb{C} \mathcal{E} \boldsymbol{u}^{0}(t): \mathcal{E} \tilde{\boldsymbol{w}} d \boldsymbol{x}
$$


for $\tilde{\boldsymbol{w}} \in V_{s_{\beta}(t)}$ for a.e. $t \in(0, T)$. Arguments identical to the Proof of Theorem 3.2 show that for $\tilde{\boldsymbol{w}} \in V_{s_{\beta}(t)}$ we have

$$
\left|I^{\epsilon_{n}}(t, \tilde{\boldsymbol{w}})\right| \leq C\|\tilde{\boldsymbol{w}}\|_{V_{s_{\beta}(t)}} .
$$

We form

$$
\int_{0}^{T} I^{\epsilon_{n}}(t, \tilde{\boldsymbol{w}}(t)) d t
$$

One then sees from Definition 3.1 that $\|\tilde{\boldsymbol{w}}(t)\|_{V_{s_{\beta}(t)}}$ is integrable and from (7.12) we can apply the Lebesgue dominated convergence theorem to conclude

$$
\lim _{\epsilon_{n} \rightarrow 0} \int_{0}^{T} I^{\epsilon_{n}}(t, \tilde{\boldsymbol{w}}(t)) d t=\int_{0}^{T} \int_{D} \mathbb{C} \mathcal{E} \boldsymbol{u}^{0}(t): \mathcal{E} \tilde{\boldsymbol{w}}(t) d \boldsymbol{x} d t .
$$

It is first noted that Lemma 3.1 can be extended in a straight forward way to the present context. Applying this to the last term in (7.1) gives

$$
-\lim _{\epsilon_{n} \rightarrow 0} \int_{0}^{T} \int_{D} \boldsymbol{b}^{\epsilon_{n}}(t) \cdot \tilde{\boldsymbol{w}}(t) d \boldsymbol{x} d t=-\int_{0}^{T} \int_{\partial D} \boldsymbol{g}(t) \cdot \tilde{\boldsymbol{w}}(t) d \sigma d t .
$$

We apply Lemma 3.2 to the first term of (7.1) and pass to a subsequence if necessary to find that

$$
\lim _{\epsilon_{n} \rightarrow 0} \rho \int_{0}^{T} \int_{D} \dot{\boldsymbol{u}}^{\epsilon_{n}}(t) \cdot \dot{\tilde{\boldsymbol{w}}}(t) d \boldsymbol{x} d t=\rho \int_{0}^{T} \int_{D} \dot{\boldsymbol{u}}^{0}(t) \cdot \dot{\tilde{\boldsymbol{w}}}(t) d \boldsymbol{x} d t .
$$

On again passing to a subsequence if necessary we recover

$$
\begin{aligned}
& -\int_{0}^{T} \rho \int_{D} \dot{\boldsymbol{u}}(t) \cdot \dot{\tilde{\boldsymbol{w}}}(t) d \boldsymbol{x} d t+\int_{0}^{T} \int_{D} \mathbb{C} \mathcal{E} \boldsymbol{u}(t): \mathcal{E} \tilde{\boldsymbol{w}}(t) d \boldsymbol{x} d t \\
& =\int_{0}^{T} \int_{\partial D} \boldsymbol{g}(t) \cdot \tilde{\boldsymbol{w}}(t) d \sigma d t
\end{aligned}
$$

where $\tilde{\boldsymbol{w}}(t)=\boldsymbol{w}\left(s_{\beta}(t)\right)=\boldsymbol{w}(t-\beta) \in V_{s_{\beta}(t)}$ for a.e. $t \in[0, T]$. Given that $\boldsymbol{w}(t) \in C_{c}^{\infty}\left(0, T ; V_{T}\right)$ we see that

$$
\lim _{\beta \rightarrow 0} \rho \int_{0}^{T} \int_{D} \dot{\boldsymbol{u}}^{0}(t) \cdot \dot{\boldsymbol{w}}(t-\beta) d \boldsymbol{x} d t=\rho \int_{0}^{T} \int_{D} \dot{\boldsymbol{u}}^{0}(t) \cdot \dot{\boldsymbol{w}}(t) d \boldsymbol{x} d t .
$$

Similarly

$$
-\lim _{\beta \rightarrow 0} \int_{0}^{T} \int_{\partial D} \boldsymbol{g}^{0}(t) \cdot \tilde{\boldsymbol{w}}(t) d \sigma d t=-\int_{0}^{T} \int_{\partial D} \boldsymbol{g}(t) \cdot \boldsymbol{w}(t) d \sigma d t .
$$

To finish the proof we show $\lim _{\beta \rightarrow 0} \boldsymbol{w}\left(s_{\beta}(t)\right)=\boldsymbol{w}(t)$ in $V_{t}$, a.e. for $t \in[0, T]$. We use the following lemma proved in [10].

Lemma 7.1. Let $\left\{V_{t}\right\}_{t \in[0, T]}$ be an increasing family of closed linear subspaces of a separable Hilbert space $V$. Then, there exists a countable set $S \subset[0, T]$ such that for all $t \in[0, T] \backslash S$, we have

$$
V_{t}=\overline{\bigcup_{s<t} V_{s}} .
$$


Observe that

$$
\bigcup_{0<\beta} V_{s_{\beta}(t)}=\bigcup_{s<t} V_{s}
$$

so $\lim _{\beta \rightarrow 0} \boldsymbol{w}\left(s_{\beta}(t)\right)=\boldsymbol{w}(t)$ in $V_{t}$, a.e. for $t \in[0, T]$, hence

$$
\lim _{\beta \rightarrow 0} \int_{D} \mathbb{C} \mathcal{E} \boldsymbol{u}^{0}(t): \mathcal{E} \tilde{\boldsymbol{w}}(t) d \boldsymbol{x}=\int_{D} \mathbb{C} \mathcal{E} \boldsymbol{u}^{0}(t): \mathcal{E} \boldsymbol{w}(t) d \boldsymbol{x} .
$$

Since $\boldsymbol{u}^{0} \in \mathcal{V}$ it is also clear from Cauchy's inequality applied to (7.11) that for $\beta>0$ that

$$
\left|\int_{D} \mathbb{C} \mathcal{E} \boldsymbol{u}^{0}(t): \mathcal{E} \tilde{\boldsymbol{w}}(t) d \boldsymbol{x}\right| \leq C\|\boldsymbol{w}(t)\|_{V_{T}}
$$

and

$$
\lim _{\beta \rightarrow 0} \int_{0}^{T} \int_{D} \mathbb{C} \mathcal{E} \boldsymbol{u}^{0}(t): \mathcal{E} \tilde{\boldsymbol{w}}(t) d \boldsymbol{x} d t=\int_{0}^{T} \int_{D} \mathbb{C} \mathcal{E} \boldsymbol{u}^{0}(t): \mathcal{E} \boldsymbol{w}(t) d \boldsymbol{x} d t
$$

follows from the Lebesgue dominated convergence theorem. Collecting results we have

$$
\begin{aligned}
& -\int_{0}^{T} \rho \int_{D} \dot{\boldsymbol{u}}(t) \cdot \dot{\boldsymbol{w}}(t) d \boldsymbol{x} d t+\int_{0}^{T} \int_{D} \mathbb{C} \mathcal{E} \boldsymbol{u}(t): \mathcal{E} \boldsymbol{w}(t) d \boldsymbol{x} d t \\
& =\int_{0}^{T} \int_{\partial D} \boldsymbol{g}(t) \cdot \boldsymbol{w}(t) d \sigma d t
\end{aligned}
$$

for all $\boldsymbol{w} \in C_{c}^{\infty}\left((0, T) ; V_{T}\right)$ with $\boldsymbol{w}(t) \in V_{t}$ and Theorem 3.4 is proved.

\section{Conclusions}

In this paper we use a double well energy within a peridynamic formulation. The advancing crack is prescribed as a softening zone followed by a failure zone in the neighborhood of a line at the center of a rectangular specimen starting from an initial crack. Symmetric forces and boundary conditions are imposed, consistent with the assumption of a crack growing on a line and moving into the specimen. The length of the failure zone and softening zone are prescribed at each time and is increasing with time. We pass to the $\epsilon \rightarrow 0$ limit along a subsequence of displacements $\boldsymbol{u}^{\epsilon}$ to find the boundary value problem satisfied by the limit displacement $\boldsymbol{u}^{0}$. The limit displacement $\boldsymbol{u}^{0}(\boldsymbol{x}, t)$ satisfies the boundary conditions of the dynamic brittle fracture problem given by

- Prescribed inhomogeneous traction boundary conditions.

- Balance of linear momentum as described by the linear elastic wave equation.

- Zero traction on the sides of the evolving crack.

- Displacement jumps can only occur inside the crack set $\Gamma_{t}$.

In this way the boundary value problem for the elastic field for dynamic Linear Elastic Fracture Mechanics (LEFM) is recovered as described in $[3,17,32,38]$. Moreover the limit displacement $\boldsymbol{u}^{0}$ is a weak solution of the wave equation on the time dependent domain $D_{t}$ containing the running crack. This establishes 
a rigorous connection between the nonlocal fracture formulation using a peridynamic model derived from a double well potential and the wave equation posed on cracking domains given in [12].

Open Access. This article is licensed under a Creative Commons Attribution 4.0 International License, which permits use, sharing, adaptation, distribution and reproduction in any medium or format, as long as you give appropriate credit to the original author(s) and the source, provide a link to the Creative Commons licence, and indicate if changes were made. The images or other third party material in this article are included in the article's Creative Commons licence, unless indicated otherwise in a credit line to the material. If material is not included in the article's Creative Commons licence and your intended use is not permitted by statutory regulation or exceeds the permitted use, you will need to obtain permission directly from the copyright holder. To view a copy of this licence, visit http://creativecommons. org/licenses/by/4.0/.

Publisher's Note Springer Nature remains neutral with regard to jurisdictional claims in published maps and institutional affiliations.

\section{References}

[1] Alicandro, R., Focardi, M., Gelli, M.S.: Finite-difference approximation of energies in fracture mechanics. Annali della Scuola Normale Superiore di Pisa 23, 671-709 (2000)

[2] Ambrosio, L., Coscia, A., Dal Maso, G.: Fine properties of functions with bounded deformation. Arch. Ration. Mech. Anal. 139, 201-238 (1997)

[3] Anderson, T.L.: Fracture Mechanics: Fundamentals and Applications, 3rd edn. Taylor \& Francis, Boca Raton (2005)

[4] Bobaru, F., Zhang, G.: Why do cracks branch? A peridynamic investigation of dynamic brittle fracture. Int. J. Fract. 196, 59-98 (2015)

[5] Bellido, J.C., Morra-Corral, C., Pedregal, P.: Hyperelasticity as a $\Gamma$-limit of peridynamics when the horizon goes to zero Calc. DOI, Var (2015). https://doi. org/10.1007/s00526-015-0839-9

[6] Borden, M., Verhoosel, C., Scott, M., Hughes, T., Landis, C.: A phase-field description of dynamic brittle fracture. Comput. Methods Appl. Mech. Eng. 217-220, 77-95 (2012)

[7] Bourdin, B., Larsen, C., Richardson, C.: A time-discrete model for dynamic fracture based on crack regularization. Int. J. Fract. 168, 133-143 (2011)

[8] Conti, S., Focardi, M., Iurlano, F.: Which special functions of bounded deformation have bounded variation? Proc. R. Soc. Edinb. Sect. A Math. 148, 33-50 (2018) 
[9] Dal Maso G., Larsen C.J., Toader R., 2020. Elastodynamic Griffith fracture on prescribed crack paths with kinks. NoDEA Nonlinear Differ. Equ. Appl. 27(1), Art. 4

[10] Dal Maso, G., Larsen, C.J.: Existence for wave equations on domains with arbitrary growing cracks. Atti. Accad. Naz. Lincei Cl. Sci. Fis. Mat. Nat. Rend. Lincei Mat. Appl. 22(9), 387-408 (2011)

[11] Dal Maso, G., Larsen, C.J., Toader, R.: Existence for constrained dynamic Griffith fracture with a weak maximal dissipation condition. J. Mech. Phys. Solids 95, 697-707 (2016)

[12] Dal Maso, G., Toader, R.: On the Cauchy problem for the wave equation on time dependent domains. J. Differ. Equ. 266, 3209-3246 (2019)

[13] Duvaut, G., Lions, J.L.: Inequalities in Mechanics and Physics. Springer, Berlin (1976)

[14] Ericksen, J.L.: Equilibrium of bars. J. Elast. 5, 191-202 (1975)

[15] Evans, L.C.: Partial Differential Equations. American Mathematical Society, Providence (1998)

[16] Evans, L.C., Gariepy, R.F.: Measure Theory and Fine Properties of Functions. CRC Press, Boca Raton (1992)

[17] Freund, B.: Dynamic Fracture Mechanics. Cambridge Monographs on Mechanics and Applied Mathematics. Cambridge University Press, Cambridge (1990)

[18] Freund, B., Clifton, R.J.: On the uniqueness of plane elastodynamic solutions for running cracks. J. Elast. 4, 293-299 (1974)

[19] Gobbino, M.: Finite difference approximation of the Mumford-Shah functional. Commun. Pure Appl. Math. 51, 197-228 (1998)

[20] Jha, P.K., Lipton, R.: Numerical analysis of nonlocal fracture models in Hölder space. SIAM J. Numer. Anal. 56(2), 906-941 (2018)

[21] Kikuchi, N., Oden, J.T.: Contact Problems in Elasticity. SIAM, Philadelphia (1988)

[22] Jha, P.K., Lipton, R.: Kinetic relations and local energy balance for LEFM from a nonlocal peridynamic model. Int. J. Fract. 226(1), 81-95 (2020)

[23] Lipton, R.: Dynamic brittle fracture as a small horizon limit of peridynamics. J. Elast. 117(1), 21-50 (2014)

[24] Lipton, R.: Cohesive dynamics and brittle fracture. J. Elast. 124(2), 143-191 (2016)

[25] Lipton, R., Said, E., Jha, P.K.: Dynamic brittle fracture from nonlocal doublewell potentials: a state-based model. In: Handbook of Nonlocal Continuum Mechanics for Materials and Structures, pp. 1-27 (2018). https://doi.org/10.1007/ 978-3-319-22977-5_33-1 
[26] Lipton, R., Said, E., Jha, P.K.: Free damage propagation with memory. J. Elast. 133(2), 129-153 (2018)

[27] Lions, J.L., Magenes, E.: Nonhomogeneous Boundary Value Problems and Applications, vol. 1. Springer, Berlin (1972)

[28] McLean, W.: Strongly Elliptic Systems and Boundary Integral Equations. Cambridge University Press, Cambridge (2000)

[29] Miehe, C., Hofacker, M., Welschinger, F.: A phase field model for rateindependent crack propagation: Robust algorithmic implementation based on operator splits. Comput. Methods Appl. Mech. Eng. 199, 2765-2778 (2010)

[30] Mengesha, T., Du, Q.: On the variational limit of a class of nonlocal functionals related to peridynamics. Nonlinearity 28(11), 3999 (2015)

[31] Nicaise, S., Sandig, A.-M.: Dynamic crack propagation in a 2D elastic body: the out-of-plane case. J. Math. Anal. Appl. 329, 1-30 (2007)

[32] Ravi-Chandar, K.: Dynamic Fracture. Elsevier, Oxford (2004)

[33] Schwab, C.H.: p- and hp- Finite Element Methods. Clarendon Press, Oxford (1998)

[34] Schmidt, B., Fraternali, F., Ortiz, M.: Eigenfracture: an eigendeformation approach to variational fracture. Multiscale Model. Simul. 7, 1237-1266 (2009)

[35] Silling, S.A.: Reformulation of elasticity theory for discontinuities and long-range forces. J. Mech. Phys. Solids 48(1), 175-209 (2000)

[36] Silling, S.A., Epton, M., Weckner, O., Xu, J., Askari, E.: Peridynamic states and constitutive modeling. J. Elast. 88(2), 151-184 (2007)

[37] Silling, S.A., Askari, E.: A meshfree method based on the peridynamic model of solid mechanics. Comput. Struct. 83, 1526-1535 (2005)

[38] Slepian, Y.: Models and Phenomena in Fracture Mechanics. Foundations of Engineering Mechanics. Springer, Berlin (2002)

[39] Trask, N., You, H., Yu, Y., Parks, M.L.: An asymptotically compatible mesh free quadrature rule for nonlocal problems with applications to peridynamics. Comput. Methods Appl. Mech. Eng. 343, 151-165 (2019)

[40] Truskinovsky, L.: Fracture as a phase transition. In: Batra, R.C., Beatty, M.F. (eds.) Contemporary Research in the Mechanics and Mathematics of Materials. CIMME, Barcelona (1996)

Robert P. Lipton

Department of Mathematics

Louisiana State University

Baton Rouge LA 70803

USA

e-mail: lipton@lsu.edu 
Prashant K. Jha

Oden Institute for Computational Engineering and Sciences

The University of Texas at Austin

Austin TX 78712

USA

e-mail:pjha@utexas.edu

Received: 18 July 2020.

Accepted: 15 February 2021. 